\title{
Quantitative study of coherent pairing modes with two-neutron transfer: Sn isotopes
}

\author{
G. Potel ${ }^{*}$ \\ Dipartimento di Fisica, Università di Milano, Via Celoria 16, 20133 Milano, Italy, \\ INFN, Sezione di Milano Via Celoria 16, 20133 Milano, Italy, Departamento de Fisica Atomica, Molecular y Nuclear, \\ Universidad de Sevilla, Facultad de Fisica, Avenida Reina Mercedes s/n, Sevilla, Spain, and \\ CEA, Centre de Saclay, IRFU, SPhN, F-91191 Gif-sur-Yvette, France
}

A. Idini $^{\dagger}$

Dipartimento di Fisica, Università di Milano, Via Celoria 16, 20133 Milano, Italy and INFN, Sezione di Milano Via Celoria 16, 20133 Milano, Italy

F. Barranco $\ddagger$

Departamento de Fisica Aplicada III, Universidad de Sevilla, Escuela Superior de Ingenieros, Sevilla, 41092 Camino de los Descubrimientos s/n, Spain

E. Vigezzi ${ }^{\S}$

INFN, Sezione di Milano Via Celoria 16, 20133 Milano, Italy

\author{
R. A. Brogliall \\ Dipartimento di Fisica, Università di Milano, Via Celoria 16, 20133 Milano, Italy, \\ INFN, Sezione di Milano Via Celoria 16, 20133 Milano, Italy, and The Niels Bohr Institute, University of Copenhagen, \\ Blegdamsvej 17, 2100 Copenhagen Ø, Denmark \\ (Received 19 September 2012; revised manuscript received 27 February 2013; published 20 May 2013)
}

\begin{abstract}
Pairing rotations and pairing vibrations are collective modes associated with a field, the pair field, which changes the number of particles by two. Consequently, they can be studied at profit with the help of two-particle transfer reactions in superfluid and in normal nuclei, respectively. The advent of exotic beams has opened, for the first time, the possibility to carry out such studies in medium heavy nuclei, within the same isotopic chain. The case studied in the present paper is that of the $\mathrm{Sn}$ isotopes [essentially from closed $(Z=N=50)$ to closed ( $Z=50, N=82$ ) shells]. The static and dynamic off-diagonal, long-range order phase coherence in gauge space displayed by pairing rotations and vibrations, respectively, leads to coherent states which behave almost classically. Consequently, these modes are amenable to an accurate nuclear structure description in terms of simple models containing the right physics, in particular, BCS plus quasiparticle random-phase approximation and Hartree-Fock mean field plus random-phase approximation, respectively. The associated two-nucleon transfer spectroscopic amplitudes predicted by such model calculations can thus be viewed as essentially "exact." This fact, together with the availability of optical potentials for the different real and virtual channels involved in the reactions considered, namely ${ }^{A+2} \mathrm{Sn}+p,{ }^{A+1} \mathrm{Sn}+d$, and ${ }^{A} \mathrm{Sn}+t$, allows for the calculation of the associated absolute cross sections without, arguably, free parameters. The numerical predictions of the absolute differential cross sections, obtained making use of the above-mentioned nuclear structure and optical potential inputs, within the framework of second-order distorted-wave Born approximation, taking into account simultaneous, successive, and nonorthogonality contributions, provide, within experimental errors in general, and below $10 \%$ uncertainty in particular, an overall account of the experimental findings for all of the measured ${ }^{A+2} \operatorname{Sn}(p, t){ }^{A} \operatorname{Sn}(\mathrm{gs})$ reactions, for which absolute cross sections have been reported to date.
\end{abstract}

DOI: 10.1103/PhysRevC.87.054321

PACS number(s): 25.40.Hs, 27.60.+j, 74.20.Fg, 74.50.+r

\section{INTRODUCTION}

Customarily, the fingerprint of shell closure in nuclei is associated with a sharp, step-function-like distinction between occupied and empty single-particle states, in correspondence

\footnotetext{
*gregory.potel@gmail.com

†andrea.idini@mi.infn.it

†barranco@us.es

§enrico.vigezzi@mi.infn.it

"Corresponding author: broglia@mi.infn.it
}

with magic numbers [1] (for a recent example concerning ${ }^{132} \mathrm{Sn}$, see Refs. [2,3]). At variance with the case of infinite, fermionic systems, in which there essentially exists a continuum of states at the Fermi energy, in finite-many-body (FMB) systems, such as, e.g., the atomic nucleus, $\varepsilon_{F}$ is not, in principle, well defined, at least not in closed-shell nuclei. This is because a sizable energy gap is observed between the last occupied $\left(j_{<}\right)$and the first unoccupied $\left(j_{>}\right)$orbitals. This is also the case for other FMB fermionic systems, e.g., $C_{60}$ fullerene, which displays a relatively large highest occupied molecular orbital (HOMO)-lowest unoccupied molecular orbital (LUMO) gap, of the order of $1.6 \mathrm{eV}$ as compared to 
$\varepsilon_{F} \approx 15 \mathrm{eV}$ [4]. In such cases, one can use as a working definition of $\varepsilon_{F}$ [5], $\left(\varepsilon_{j_{>}}+\varepsilon_{j_{<}}\right) / 2\left(\left(\varepsilon_{\mathrm{HOMO}}+\varepsilon_{\text {LUMO }}\right) / 2\right.$ in the case of $\left.C_{60}\right)$. Away from closed shells, medium-heavy nuclei become, as a rule, superfluid, the distinction between occupied and empty states being blurred around $\varepsilon_{F}$. The Fermi energy is, in such situation, well defined and equal to the energy for which the occupancy probability attains the value of one half. In keeping with this result, in the case of closed-shell nuclei, $\varepsilon_{F}$ can be properly defined as the minimum of the dispersion relation associated with pair addition and pair removal modes.

\section{PAIR-SPIN FORMALISM}

The mixing taking place in superfluid nuclei between particle and holes is economically embodied in the BogoliubovValatin [6,7] quasiparticle transformation

$$
\begin{aligned}
& \alpha_{v}=U_{\nu} a_{v}-V_{\nu} a_{\bar{v}}^{\dagger}=U_{v}^{\prime} a_{v}^{\prime}-V_{\nu}^{\prime} a_{\bar{v}}^{\prime \dagger}, \\
& \alpha_{\nu}^{\dagger}=U_{v}^{*} a_{v}^{\dagger}-V_{v}^{*} a_{\bar{v}}=U_{v}^{\prime} a_{v}^{\prime \dagger}-V_{v}^{\prime} a_{\bar{v}}^{\prime},
\end{aligned}
$$

where

$$
U_{\nu}=U_{\nu}^{\prime} e^{i \phi}, \quad V_{\nu}=V_{\nu}^{\prime} e^{-i \phi},
$$

are the BCS occupation amplitudes, $U_{v}^{\prime}$ and $V_{v}^{\prime}$ being real quantities, while $a_{\bar{\nu}}^{\prime \dagger}=\mathcal{G}(\phi) a_{\bar{\nu}}^{\dagger} \mathcal{G}^{-1}(\phi)=e^{-i \phi} a_{\bar{\nu}}^{\dagger}$ and $a_{\bar{\nu}}^{\prime}=$ $\mathcal{G}(\phi) a_{\bar{v}} \mathcal{G}^{-1}(\phi)=e^{i \phi} a_{\bar{v}}$ are creation and annihilation operators, respectively, referred to the intrinsic system of reference in gauge space (i.e., body-fixed BCS deformed state), $\mathcal{G}=$ $\exp (-i N \phi)$ inducing a rotation of the angle $\phi$ in this [twodimensional (2D)] space (gauge transformation), with $N$ being the number of particle operator (see Appendixes A-C). The states $|v\rangle$ and $|\bar{v}\rangle$, connected by the time-reversal operator, have the same energy (Kramers degeneracy). In keeping with the fact that (1) is a unitary transformation,

$$
U_{\nu} U_{v}^{*}+V_{\nu} V_{v}^{*}=U_{v}^{\prime 2}+V_{v}^{\prime 2}=1 .
$$

The transformation (1) provides the rotation in Hilbert space of the creation and annihilation fermion operators, which diagonalizes the mean-field, BCS pairing Hamiltonian [angle $\theta_{v}$; see Figs. 11(b) and 11(c)],

$$
\begin{aligned}
\left(H_{p}-\lambda N\right)_{\mathrm{MF}} & =\sum_{\nu>0} \epsilon_{\nu} N_{\nu}-G \alpha_{0}^{\prime}\left(P^{\prime \dagger}+P^{\prime}\right)+G \alpha_{0}^{\prime 2} \\
& =\sum_{\nu>0} E_{\nu} \tilde{N}_{\nu}+E_{\mathrm{gs}},
\end{aligned}
$$

where, $\epsilon_{v}=\varepsilon_{v}-\lambda$ and

$$
E_{\mathrm{gs}}=\sum_{v>0}\left(\epsilon_{v}-E_{v}\right)+\frac{\Delta^{\prime 2}}{G},
$$

is the ground-state energy (see Appendix A), while $\lambda=\varepsilon_{F}$. In the above expression we find the particle number operators,

$$
N_{\nu}=a_{\nu}^{\dagger} a_{v}+a_{\bar{\nu}}^{\dagger} a_{\bar{\nu}},
$$

the pair creation and annihilation operators,

$$
P_{\nu}^{\dagger}=a_{\nu}^{\dagger} a_{\bar{v}}^{\dagger}, \quad P_{v}=a_{\bar{v}} a_{v},
$$

TABLE I. Results for the valence shell lying closer to Fermi energy, of a BCS calculation, for ${ }^{120} \mathrm{Sn}$. The mean field used corresponds to a Saxon-Woods potential, in a 15-fm spherical box (continuum discretization). The pairing coupling constant used, $G=0.05 \mathrm{MeV}$ leads to the value of pairing gap obtained from the three-point formula $\left(\Delta^{\prime}=1.47 \mathrm{MeV}\right)$, summing the contributions of states from the $1 s_{1 / 2}$ at -39 to $+60 \mathrm{MeV}$. The resulting Fermi energy computed by solving the BCS number equation is $\lambda=-6.9 \mathrm{MeV}$. The quantity $\alpha_{0}^{\prime}=\sum_{v>0} U_{v}^{\prime} V_{v}^{\prime}\left(=\sum_{j} \sum_{m>0} U_{j}^{\prime} V_{j}^{\prime}=\sum_{j} \Omega_{j} U_{j}^{\prime} V_{j}^{\prime}\right)$ $\approx 6.1$ for the valence-shell space and $\alpha_{0}^{\prime} \approx 29.4$ for the whole single-particle space used in the calculation.

\begin{tabular}{lccccccc}
\hline \hline & $\varepsilon_{v}$ & $\epsilon_{v}$ & $E_{v}$ & $U_{v}^{\prime}$ & $V_{v}^{\prime}$ & $U_{v}^{\prime} V_{v}^{\prime}$ & $\Omega_{v} U_{v}^{\prime} V_{v}^{\prime}$ \\
\hline$d_{5 / 2}$ & -9.21 & -2.31 & 2.72 & 0.28 & 0.96 & 0.27 & 0.81 \\
$g_{7 / 2}$ & -8.70 & -1.80 & 2.31 & 0.34 & 0.94 & 0.32 & 1.28 \\
$s_{1 / 2}$ & -7.42 & -0.52 & 1.55 & 0.58 & 0.81 & 0.47 & 0.47 \\
$d_{3 / 2}$ & -6.98 & -0.08 & 1.47 & 0.69 & 0.72 & 0.50 & 1.00 \\
$h_{11 / 2}$ & -5.97 & 0.93 & 1.75 & 0.88 & 0.48 & 0.42 & 2.52 \\
$f_{7 / 2}$ & -1.87 & 5.03 & 5.26 & 0.99 & 0.14 & 0.14 & 0.56 \\
$p_{3 / 2}$ & -0.78 & 6.12 & 6.32 & 0.99 & 0.12 & 0.12 & 0.24 \\
\hline \hline
\end{tabular}

the complex ( $\phi$ indicating the gauge angle) condensed (superfluid) Cooper field (see Appendix B),

$$
\begin{aligned}
\alpha_{0} & =\langle\mathrm{BCS}|P| \mathrm{BCS}\rangle=\left\langle\mathrm{BCS}\left|P^{\dagger}\right| \mathrm{BCS}\right\rangle^{*}=e^{-2 i \phi} \alpha_{0}^{\prime} \\
& =e^{-2 i \phi} \sum_{\nu>0} U_{\nu}^{\prime} V_{\nu}^{\prime}=\sum_{\nu>0} U_{\nu}^{*} V_{\nu},
\end{aligned}
$$

the quasiparticle energy,

$$
E_{v}=\left(\epsilon_{v}^{2}+\Delta^{\prime 2}\right)^{1 / 2},
$$

the absolute value (modulus) of the pairing gap,

$$
\Delta^{\prime}=G \alpha_{0}^{\prime}=G \sum_{\nu>0} U_{v}^{\prime} V_{\nu}^{\prime}=G e^{2 i \phi} \sum_{\nu>0} U_{v}^{*} V_{\nu}=\Delta e^{2 i \phi},
$$

and the quasiparticle number operators,

$$
\tilde{N}_{\nu}=\alpha_{\nu}^{\dagger} \alpha_{\nu}+\alpha_{\bar{\nu}}^{\dagger} \alpha_{\bar{\nu}} .
$$

For the case of ${ }^{120} \mathrm{Sn}$, the quantities $\epsilon_{v}, E_{v}, U_{v}^{\prime}, V_{v}^{\prime}$, and $U_{v}^{\prime} V_{v}^{\prime}$ introduced above are given in Tables I and II.

The ground state of the system referred to the laboratory system of reference $\mathcal{K}$, as well as to the intrinsic (body-fixed) frame $\mathcal{K}^{\prime}$ (see Appendixes A and B), can be written as

$$
\begin{aligned}
|\operatorname{BCS}(\phi)\rangle_{\mathcal{K}} & =\frac{1}{\operatorname{Norm}} \prod_{\nu>0} \alpha_{\nu} \alpha_{\bar{v}}|0\rangle=\prod_{\nu>0}\left(U_{v}+V_{\nu} a_{\nu}^{\dagger} a_{\bar{\nu}}^{\dagger}\right)|0\rangle \\
& =\prod_{\nu>0} e^{i \phi}\left(U_{\nu}^{\prime}+V_{\nu}^{\prime} e^{-2 i \phi} a_{\nu}^{\dagger} a_{\bar{\nu}}^{\dagger}\right)|0\rangle \\
& =e^{i \Omega \phi} \prod_{\nu>0}\left(U_{\nu}^{\prime}+V_{\nu}^{\prime} a_{\nu}^{\prime \dagger} a_{\bar{\nu}}^{\prime \dagger}\right)|0\rangle \\
& =e^{i \Omega \phi}|\operatorname{BCS}(\phi=0)\rangle_{\mathcal{K}^{\prime}},
\end{aligned}
$$

where $\Omega$ is the pair degeneracy of the single-particle subspace, leading to an overall (trivial) phase.

The central feature of the $|\mathrm{BCS}\rangle$ wave function, that is condensation of largely overlapping Cooper pairs, is captured by the pairspin (quasispin) formulation of superconductivity 
TABLE II. Results for the valence shell lying closer to Fermi energy, of a BCS calculation, for ${ }^{120} \mathrm{Sn}$. The mean field used corresponds to a Saxon-Woods potential, in a 15-fm spherical box (continuum discretization). The pairing coupling constant used, $G=0.18 \mathrm{MeV}$ leads to the value of pairing gap obtained from the three-point formula ( $\Delta=1.47 \mathrm{MeV})$, summing the contributions of states from the $1 s_{1 / 2}$ at -39 to $+0 \mathrm{MeV}$. The resulting Fermi energy computed by solving the BCS number equation is $\lambda=-6.72 \mathrm{MeV}$. The quantity $\alpha_{0}^{\prime} \approx 6.1$ (see caption to Table I) for the valence-shell space and $\alpha_{0}^{\prime} \approx 8.2$ for the whole single-particle space.

\begin{tabular}{lccccccc}
\hline \hline & $\varepsilon_{v}$ & $\epsilon_{v}$ & $E_{v}$ & $U_{v}^{\prime}$ & $V_{v}^{\prime}$ & $U_{v}^{\prime} V_{v}^{\prime}$ & $\Omega_{v} U_{v}^{\prime} V_{v}^{\prime}$ \\
\hline$d_{5 / 2}$ & -9.21 & -2.45 & 2.90 & 0.26 & 0.96 & 0.25 & 0.75 \\
$g_{7 / 2}$ & -8.70 & -1.98 & 2.48 & 0.32 & 0.95 & 0.30 & 1.20 \\
$s_{1 / 2}$ & -7.42 & -0.70 & 1.63 & 0.54 & 0.84 & 0.45 & 0.45 \\
$d_{3 / 2}$ & -6.98 & -0.26 & 1.49 & 0.64 & 0.77 & 0.49 & 0.98 \\
$h_{11 / 2}$ & -5.97 & 0.75 & 1.64 & 0.85 & 0.53 & 0.45 & 2.70 \\
$f_{7 / 2}$ & -1.88 & 4.84 & 5.04 & 0.99 & 0.15 & 0.15 & 0.60 \\
$p_{3 / 2}$ & -0.78 & 5.94 & 6.10 & 0.99 & 0.12 & 0.12 & 0.24 \\
\hline \hline
\end{tabular}

(see Refs. [8,9] and references therein), which adscribes values [see Fig. 1(a) and Appendix A ]

$$
\left\langle s_{x}(v)\right\rangle=\left\langle s_{y}(v)\right\rangle=0, \quad s_{z}(v)=-1 / 2,
$$

to empty states, and

$$
\left\langle s_{x}(v)\right\rangle=\left\langle s_{y}(v)\right\rangle=0, \quad s_{z}(v)=+1 / 2,
$$

to occupied states of the noninteracting (normal) system. The symmetry axis in pairspin space (the $z$ axis) is referred to as the gauge axis [see Appendix A, Fig. 11(a)]. A superfluid system [see Fig. 1(b); see also Fig. 13 Appendix A] is characterized by a collective pairspin $\overrightarrow{S_{\perp}} \equiv\left\{S_{x}, S_{y}\right\}$, which points in a direction perpendicular to $z$, associated with the azimuthal angle $2 \phi$. This direction defines an intrinsic reference frame $\mathcal{K}^{\prime}$ (see Fig. 14, Appendix B), in which the components of the average total pairspin $\langle\vec{S}\rangle=\sum_{v>0}\langle\vec{s}(v)\rangle$ in the mean-field ground state take the values [see Fig. 11(b) and Appendix A; see also Eqs. (B28a) and (B29a)]

$$
\begin{aligned}
& \left\langle S_{y}^{\prime}\right\rangle=0, \\
& \left\langle S_{x}^{\prime}\right\rangle=\alpha_{0}^{\prime}=\sum_{v>0} U_{v}^{\prime} V_{v}^{\prime}, \\
& \left\langle S_{z}\right\rangle \equiv\left\langle S_{z}^{\prime}\right\rangle=\frac{1}{2} \sum_{v>0}\left(V_{v}^{\prime 2}-U_{v}^{\prime 2}\right) .
\end{aligned}
$$

Thus, $S_{\perp}$ gives a measure of the mixing of empty and occupied states of the BCS solution [see Eqs. (13) and (14)]. This is tantamount to saying that $S_{\perp}$ defines a privileged orientation in gauge space (see Appendix A), in keeping with the fact that Eq. (12) is a wave packet in the number of pairs of particles. An emergent property of the associated symmetry-breaking phase transition is generalized rigidity in gauge space (see Appendix C). That is, the system can be set into rotation (or change its rotational frequency) in gauge space in terms of two-particle transfer reactions. The pairspin polarization may rotate collectively around the gauge axis, and the azimuthal angle $2 \phi$ of $S_{\perp}$ [see Appendix A and Fig. 11; see
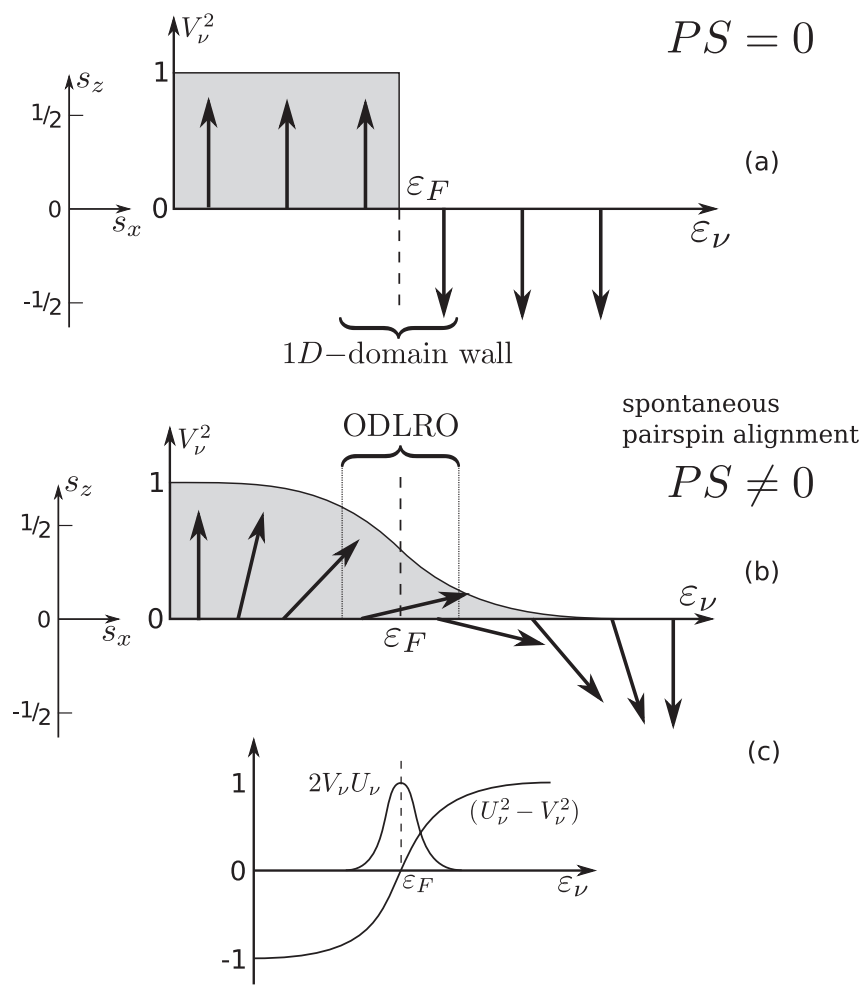

FIG. 1. (a) The occupancy $\left\langle N_{v}\right\rangle \sim V_{v}^{\prime 2}$ of the single-particle states of the unperturbed system, in which the individual pairspins are aligned along the gauge $(z)$ axis (see Fig. 11). This noncorrelated system $\left(\alpha_{0}^{\prime}=0\right)$ displays zero pairspin alignment $(P S=0)$, that is $\left\langle S_{x}\right\rangle=0$. (b) The superconducting (nucleon superfluid) ground state displays off-diagonal long-range order [ODLRO, see Eq. (A32)] and a finite value of the total pairspin $\left(P S \neq 0 ; \alpha_{0}^{\prime}=\sum_{v>0} U_{v}^{\prime} V_{v}^{\prime}\right)$, i.e., $\left\langle s_{x}(v)\right\rangle \neq 0$, can be viewed as a one-dimensional domain wall (see also Fig. 13 of Appendix A). (c) Amplitudes with which $z$ and $x$ components of the pairspin mix, leading to a privileged orientation in gauge space perpendicular to the gauge $(z)$ axis [see Fig. 11(b)].

also Eqs. (B28a) and (B29a)] is therefore a dynamical variable associated with pairing rotational bands.

\section{A. Order parameter}

The modulus of the order parameter (8), that is, of the quantity $\alpha_{0}^{\prime}=\sum_{v>0} U_{v}^{\prime} V_{v}^{\prime}=\Delta^{\prime} / G$, is, for medium heavy nuclei $(A \approx 120)$ of the order of $1.4 \mathrm{MeV} / G \approx 7$, in keeping with the fact that $G \approx 25 \mathrm{MeV} / A$ (major $j$-shell approximation; see Ref. [10], Chapters 2 and 3, and references therein). In other words, roughly of the order of ten $(v, \bar{v})$ Cooper pairs contribute to the nuclear condensate in superfluid nuclei. Consequently, large fluctuations are expected for $\alpha_{0}^{\prime}$.

These fluctuations are generated by the residual interaction acting between the quasiparticles (cf. Ref. [8], cf. also Ref. [10], in particular Chap. 4 and Appendixes I and J of this reference). In the harmonic (quasiparticle random phase) approximation the two associated pair fields are: (1) $\left(U_{v}^{\prime 2}-V_{v}^{\prime 2}\right)\left(\Gamma_{v}^{\dagger}+\Gamma_{v}\right)$, leading essentially to a bound two-quasiparticle-like state (pairing vibration mixed to $\beta$ vibrations, in deformed nuclei; cf. Ref. [11] and references therein) lying on top of the pairing gap, $\Gamma_{\nu}^{\dagger}=\alpha_{\nu}^{\dagger} \alpha_{\bar{\nu}}^{\dagger}$ being 


\section{Spontaneous Symmetry Breaking II}

I

3D-space

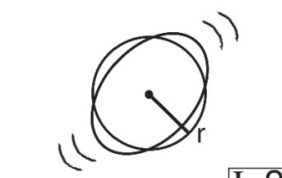

$\mathrm{I}=2$

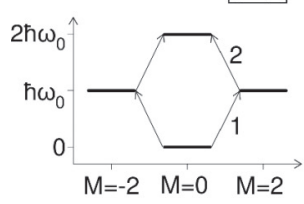

$\mathrm{N}$ gauge space

$$
\text { Dynamic }
$$$$
\omega_{0}=\sqrt{C / D}
$$

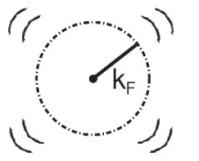

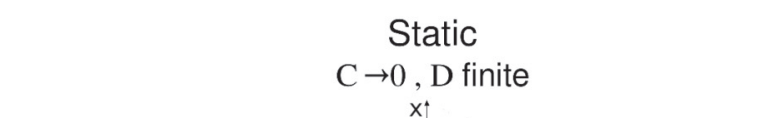

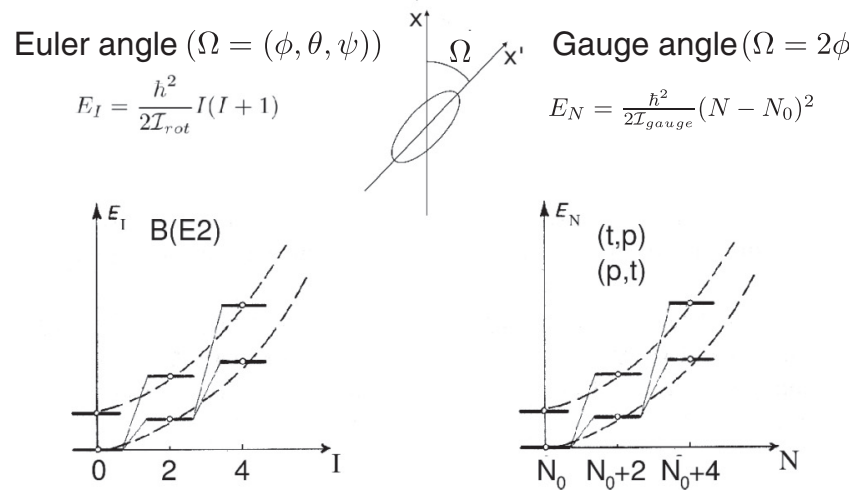

FIG. 2. Schematic representation of the nuclear structure consequences of spontaneous symmetry breaking of rotational and of gauge invariance (see also Table XI of Ref. [12]).

the two-quasiparticle creation quasiboson operator; (2) $\left(U_{v}^{\prime 2}+\right.$ $\left.V_{v}^{\prime 2}\right)\left(\Gamma_{v}^{\dagger}-\Gamma_{v}\right)$, which sets the $|\mathrm{BCS}\rangle_{\mathcal{K}^{\prime}}$ intrinsic state into rotation in gauge space and whose fluctuations diverge in the long-wavelength limit, in just such a way that the resulting ground state,

$$
\left|N_{0}\right\rangle \sim \int \mathrm{d} \phi e^{i N_{0} \phi}|\mathrm{BCS}(\phi)\rangle_{\mathcal{K}} \sim\left(\sum_{\nu>0} c_{\nu} a_{\nu}^{\dagger} a_{\bar{\nu}}^{\dagger}\right)^{N_{0} / 2}|0\rangle,
$$

transforms irreducibly under gauge transformation

$$
\mathcal{G}(\phi)=e^{-i N \phi} \text {. }
$$

The states (16) are the members of a pairing rotational band built out of a condensation of $N_{0} / 2$ Cooper pairs each described by $|\tilde{0}\rangle=\sum_{\nu>0} c_{\nu} a_{\nu}^{\dagger} a_{\bar{\nu}}^{\dagger}|0\rangle$ [see Appendix B, in particular Eqs. (B16), (B17), and (B23)]. It is of notice that the presence of rotational bands in the spectra of many-body systems is the fingerprint of deformation both in real (3D) and abstract (e.g., gauge) spaces (see Fig. 2; see also Table XI in Ref. [12]).

Fluctuations of the pair field are, of course, already present in the normal (correlated) ground state $|0\rangle=\left|\mathrm{gs}\left(A_{0}\right)\right\rangle$ of closed-shell nuclei (mass number $A_{0}$ ). That is, in systems in which, while $\alpha_{0}^{\prime}=0$, the (dynamical) value of the order parameter, i.e., the zero-point fluctuation of $\alpha_{0}^{\prime}$ around its zero value,

$$
\begin{aligned}
\alpha_{\text {dyn }}^{2} & =\left\langle\left(\alpha-\alpha_{0}^{\prime}\right)^{2}\right\rangle=1 / 2\left(\left\langle 0\left|P^{\dagger} P\right| 0\right\rangle+\left\langle 0\left|P P^{\dagger}\right| 0\right\rangle\right) \\
& =\frac{1}{2}\left(\sum_{\text {int }}\left|\left\langle\operatorname{int}\left(A_{0}-2\right)|P| 0\right\rangle\right|^{2}+\left|\left\langle\operatorname{int}\left(A_{0}+2\right)\left|P^{\dagger}\right| 0\right\rangle\right|^{2}\right) \\
& \approx \frac{1}{2}\left(\left|\left\langle\operatorname{gs}\left(A_{0}-2\right)|P| 0\right\rangle\right|^{2}+\left|\left\langle\operatorname{gs}\left(A_{0}+2\right)\left|P^{\dagger}\right| 0\right\rangle\right|^{2}\right), \quad(18)
\end{aligned}
$$

where "int" denotes a basis of intermediate states in the nuclei $A_{0}+2$ and $A_{0}-2$, displays finite values (see Refs. [11,13-15] and references therein), directly related to the quantity $E_{\text {corr }} / G$, where $E_{\text {corr }}$ is the average correlation energy of the pair addition $\left[\left|g s\left(A_{0}+2\right)\right\rangle\right.$ state] and of the pair removal $\left[\left|g s\left(A_{0}-2\right)\right\rangle\right.$ state $]$ modes, in other words, the binding energy of the two-nucleon Cooper pair moving on top of the $A_{0}$ closed-shell system and of the two-hole Cooper pair moving in the $A_{0}$ Fermi sea.

In the case of ${ }^{132} \mathrm{Sn}\left(A_{0}=132\right)$, we obtain (see Table III and Fig. 3)

$$
\begin{aligned}
& E_{\mathrm{corr}}\left(A_{0}+2\right)=2\left|\varepsilon_{j>}-\lambda\right|-W\left(A_{0}+2\right)=1.17 \mathrm{MeV}, \\
& E_{\mathrm{corr}}\left(A_{0}-2\right)=2\left|\varepsilon_{j<}-\lambda\right|-W\left(A_{0}-2\right)=2.14 \mathrm{MeV},
\end{aligned}
$$

and thus $\bar{E}_{\text {corr }}=1.66 \mathrm{MeV}$. It is of notice that in the case in which the energies $W \rightarrow 0$, the system becomes superfluid, the BCS $\lambda$ parameter coinciding with the minimum value of the dispersion relation shown in Fig. 3(a). Consequently, $E_{\text {corr }} / G \approx \frac{1}{2}\left[\frac{E_{\text {corr }}\left(A_{0}+2\right)}{G\left(A_{0}+2\right)}+\frac{E_{\text {corr }}\left(A_{0}-2\right)}{G\left(A_{0}-2\right)}\right] \approx \frac{1}{2}(8.9+13.6) \approx 11$, a value which is not very different from that of $\Delta^{\prime} / G$ associated with the superfluid nucleus ${ }^{120} \mathrm{Sn}$.

The quantity $\alpha_{\mathrm{dyn}}$ can be also estimated from the ratio $(\Lambda / G)$ between the (two-particle)-(two-hole) (pairing) vibration coupling strength (see, e.g., Ref. [11]) and the pairing coupling constant. In the two-level model [10], this quantity is given by $(\Lambda / G)=2 \sqrt{\Omega}, \Omega$ being the pair degeneracy of the single-particle space in which the two nucleons (two nucleon holes) participating in the pair addition and removal modes are allowed to correlate. As a rule, these are the valence shells of the closed-shell system $A_{0}$.

In the case of the newly discovered ${ }^{132} \mathrm{Sn}$, doubly magic nucleus, $\Omega \approx 30$ and $\alpha_{\text {dyn }} \approx(\Lambda / G) \approx 2 \sqrt{30} \approx 11$. Making use of the actual values of $\Lambda$ (see Table III), one obtains $\frac{1}{2}\left[\frac{1.08}{0.131}+\frac{1.60}{0.157}\right] \approx 9$. Summing up, $\alpha_{\text {dyn }}\left({ }^{132} \mathrm{Sn}\right) \geqslant \alpha\left({ }^{120} \mathrm{Sn}\right)$.

This result embodies the very difference between gauge spontaneous symmetry breaking in atomic nuclei and in condensed matter. In a chunk of, e.g., $\mathrm{Pb}$, of which more than $50 \%$ is the atom built on the isotope ${ }^{208} \mathrm{~Pb}$ (or of $\mathrm{Sn}$, of which none is the atom built on the isotope ${ }^{132} \mathrm{Sn}$, the corresponding nucleus being highly unstable), at a temperature below the critical temperature $T_{c}=7.2 \mathrm{~K}(3.72 \mathrm{~K})$ at which the metal becomes superconducting but in the presence of a magnetic field stronger than the critical value $H_{c}=0.08 \mathrm{~T}(0.03 \mathrm{~T})$, of the order of $10^{3}$ times Earth's magnetic field, Cooper pairs break as soon as they are formed, leading to a hardly observable 
TABLE III. RPA wave functions of pair addition and removal mode of ${ }^{132} \mathrm{Sn}$ (above) and ${ }^{100} \mathrm{Sn}$ (below). Single-particle energies have been taken from experimental values referenced in the National Nuclear Data Center. The energy of the lowest pairing addition and removal phonons in ${ }^{132} \mathrm{Sn}$ are, respectively, $W\left(A_{0}+2\right)=3.45 \mathrm{MeV}$ with $G\left(A_{0}+2\right)=0.131 \mathrm{MeV}$ and $W\left(A_{0}-2\right)=3.06 \mathrm{MeV}$ with $G\left(A_{0}-2\right)=0.157 \mathrm{MeV}$, the associated particle-pair vibration coupling strength [see Eq. (20)] being $\Lambda_{\text {add }}=\Lambda\left(A_{0}+2\right)=1.08 \mathrm{MeV}$ and $\Lambda_{\text {rem }}=\Lambda\left(A_{0}-2\right)=1.61 \mathrm{MeV}$, respectively. The minimum of the dispersion relation, and thus the Fermi energy, are equal to $\lambda=-4.75 \mathrm{MeV}$ [see Fig. 3(a)]. The pair degeneracy of the single-particle space associated with ${ }^{132} \mathrm{Sn}$ and ${ }^{100} \mathrm{Sn}$ is $\Omega=31$ and $\Omega=27$, respectively. The energy of the lowest pairing addition and removal phonons in ${ }^{100} \mathrm{Sn}$ are, respectively, $W\left(A_{0}+2\right)=5.13 \mathrm{MeV}$ with $G\left(A_{0}+2\right)=0.290 \mathrm{MeV}$ and $W\left(A_{0}-2\right)=1.96 \mathrm{MeV}$ with $G\left(A_{0}-2\right)=0.380 \mathrm{MeV}$, the Fermi energy being $\lambda=-14.5 \mathrm{MeV}$ [see Fig. 3(b)]. The associated $\Lambda$ values being $\Lambda_{\text {add }}=2.72 \mathrm{MeV}$ and $\Lambda_{\text {rem }}=5.32 \mathrm{MeV}$. The binding energy of ${ }^{98} \mathrm{Sn}$ was assumed to be $B\left({ }^{98} \mathrm{Sn}\right)=794.24 \mathrm{MeV}$, from the polynomial (fourth grade) fit of the binding energies of tin isotopic chain.

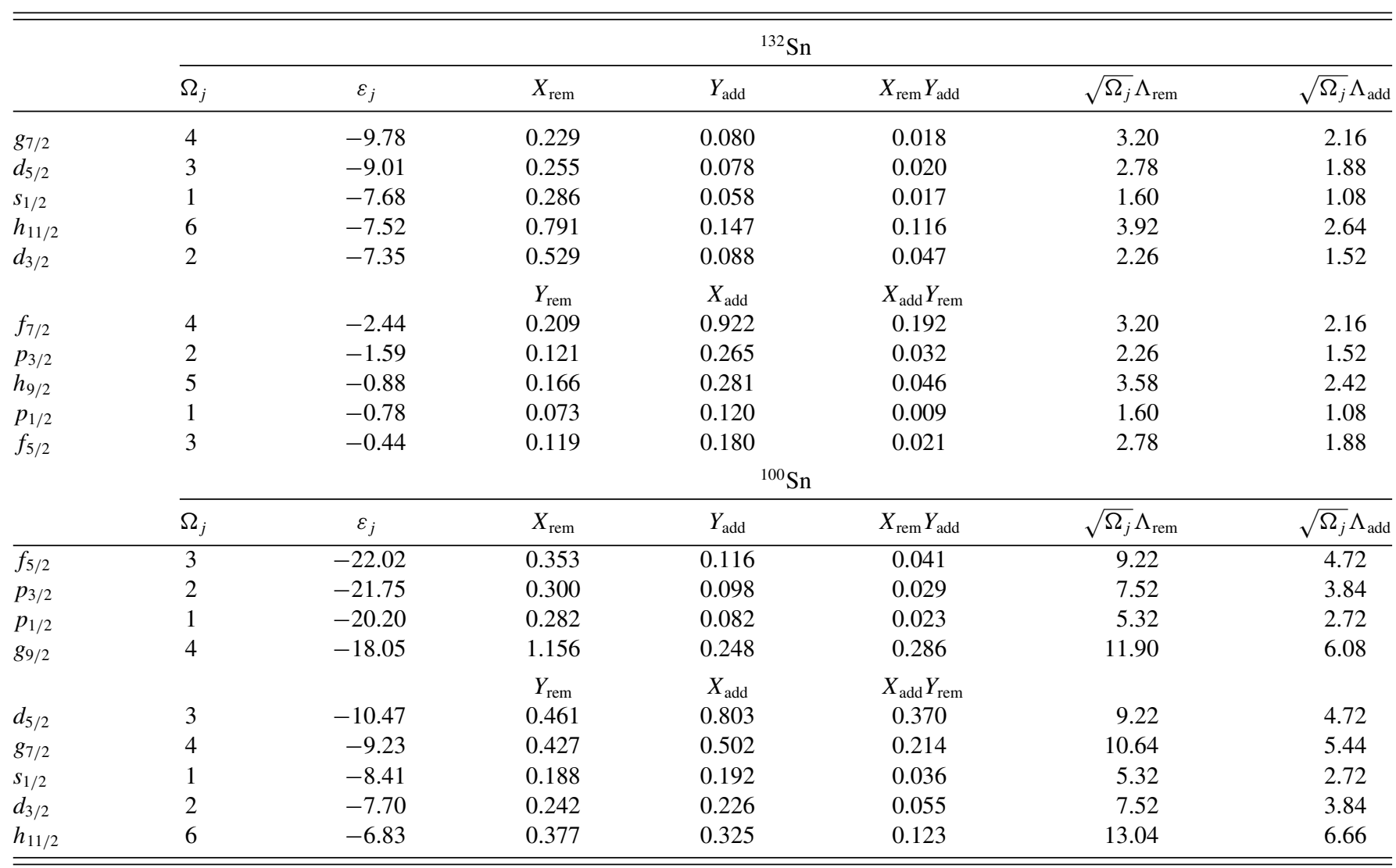

effect, in particular concerning the structure and stability of the crystal.

However, in the case of their ground state and thus at absolute zero temperature - as is the case for all natural occurring nuclear species on Earth - the Cooper pairs associated with the normal (nonsuperfluid) system displaying two nucleons above or two holes below closed shell, such as, e.g., $\left|\mathrm{gs}\left({ }_{82}^{210} \mathrm{~Pb}_{128}\right)\right\rangle$ and $\left|\mathrm{gs}\left({ }_{82}^{206} \mathrm{~Pb}_{124}\right)\right\rangle$, respectively, these fermions are strongly correlated, as evidenced by the large two-nucleon transfer cross sections with which they are excited (see, e.g., Ref. [12] and references therein). In keeping with the fact that a consistent fraction of this cross section arises, e.g., in the case of ${ }^{208} \mathrm{~Pb}(t, p){ }^{210} \mathrm{~Pb}$ (gs) by the transfer of two particles to levels below the Fermi energy of ${ }^{208} \mathrm{~Pb}$ (ground-state correlations), Cooper pair correlations blur dynamically the difference between occupied and empty single-particle states thought to be a trademark of closed-shell systems.

The same arguments presented above can be used for ${ }_{50}^{132} \mathrm{Sn}_{82}$, in which case the summed backwardsgoing amplitudes amount to $\sum_{i} Y_{i} \approx 0.5$, as reported in Table III. The forwardsgoing and backwardsgoing amplitudes are given by

$$
\left.\begin{array}{l}
X_{n}(j ; \beta) \\
Y_{n}(j ; \beta)
\end{array}\right\}=\frac{\left(\sqrt{\Omega_{j}} / 2\right) \Lambda_{n}(\beta)}{2\left|\epsilon_{j}\right| \mp W_{n}(\beta)} .
$$

The quantity $\Omega_{j}=(2 j+1) / 2$ is the pair degeneracy of orbital $j, \beta=+2$ and $\beta=-2$ label the pair addition and pair removal modes, respectively, while $n(=1,2, \ldots)$ numbers the solutions of the RPA dispersion relation in subsequent order of excitation energy (we deal here only with the $n=1$, lowest energy pairing vibrations). The $-(+)$ sign in Eq. (20) refers to $X$ and $Y$ amplitudes respectively. The $X(j)(Y(j))$ amplitudes in Eq. (20) are associated with particle (hole) states respectively, when $\beta=+2$; and with hole (particle) states, when $\beta=$ -2 . We define $X_{\text {add }}(j) \equiv X_{1}(j ; 2) ; Y_{\text {add }}(j) \equiv Y_{1}(j ; 2)$ and $X_{\text {rem }}(j) \equiv X_{1}(j ;-2) ; Y_{\text {rem }}(j) \equiv Y_{1}(j ;-2)$. The amplitudes $Y_{\text {rem }}(j)$ and $Y_{\text {add }}(j)$ are directly related to the population of particle states and depopulation of hole states respectively. In particular, the renormalized values of the number of particles 


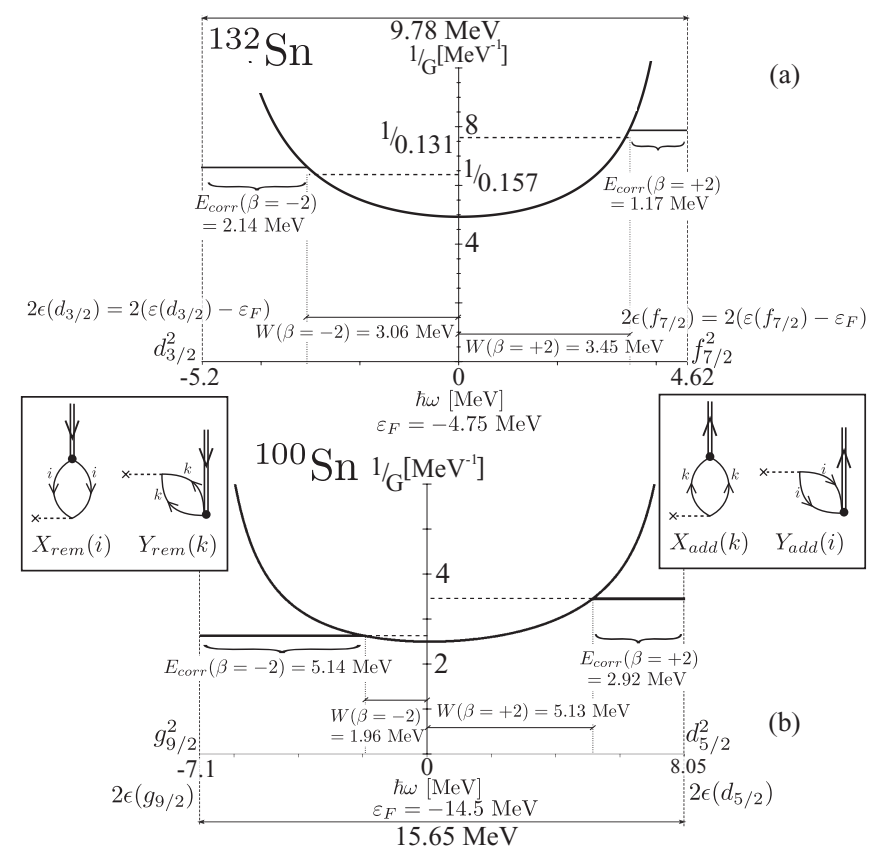

FIG. 3. Dispersion relation [see Ref. [11]; see also [10] Eq. (5.50)] associated with the pairing vibration of ${ }^{132} \mathrm{Sn}(\mathrm{a})$ and of ${ }^{100} \mathrm{Sn}(\mathrm{b})$. (a) The part of the curve to the left of the minimum corresponds to the pair removal mode $\left(\left|\mathrm{gs}\left({ }^{130} \mathrm{Sn}\right)\right\rangle\right)$ while that to the right is associated with the pair addition mode $\left[\left|\operatorname{gs}\left({ }^{134} \mathrm{Sn}\right)\right\rangle\right]$. The energy of the modes $W\left(A_{0} \pm 2\right)$ are measured from the minimum of the dispersion relation, its values being explicitly indicated (see also caption to Table II). The coupling constants $G\left(A_{0} \pm 2\right)$ used in the calculations are given in the caption of Table II, where the $X$ and $Y$ amplitudes of the corresponding wave functions are displayed. As seen from the insets, the pairing vibrational modes blur the sharp distinction between occupied and empty states. In fact, the pair addition mode can be excited not only by transferring two neutrons to levels lying above the Fermi energy, a process proportional to the $X_{\text {add }}(k)=\frac{\sqrt{\left(\Omega_{j_{k}} / 2\right) \Lambda_{\text {add }}}}{2 \epsilon_{k}-W_{\text {add }}}$ amplitude (inset to the right), but also to states lying below the Fermi energy, a process proportional to $Y_{\text {add }}(i)=\frac{\sqrt{\left(\Omega_{j_{i}} / 2\right) \Lambda_{\text {add }}}}{2\left|\epsilon_{i}\right|+W_{\text {add }}}$. The associated values of the particle-pairing vibration coupling strength are $\Lambda_{\text {add }}=\Lambda(\beta=+2)=1.08 \mathrm{MeV}$ and $\Lambda_{\text {rem }}=\Lambda(\beta=$ $-2)=1.6 \mathrm{MeV}$. (b) The same as above, but for the closed-shell system ${ }^{100} \mathrm{Sn}$. In this case $\Lambda_{\text {add }}=2.72 \mathrm{MeV}$ and $\Lambda_{\text {rem }}=5.32 \mathrm{MeV}$.

on a given pair are

$$
\begin{aligned}
& \left\langle N_{\nu}\right\rangle=2 Y_{\mathrm{rem}}^{2}\left(j_{v}\right) / \Omega_{j_{v}}, \quad \epsilon_{j_{v}}>0 ; \\
& \left\langle N_{\nu}\right\rangle=1-2 Y_{\text {add }}^{2}\left(j_{v}\right) / \Omega_{j_{v}}, \quad \epsilon_{j_{v}} \leqslant 0 .
\end{aligned}
$$

Arguably, one can posit that in the nuclear case it is not, or at least not only, the condensed (superfluid) state that is peculiar, but the normal state,$^{1}$ in which pair addition

\footnotetext{
${ }^{1}$ Within this context one can also mention a different (although not directly pertinent to the $\mathrm{Sn}$ isotopes studied in the present paper) analogy between nuclear and metallic normal-state properties that has important consequences on Cooper pair stability. Bad conductors, that is, bad single-particle electronic metals (such as, e.g., $\mathrm{Pb}, \mathrm{Sn}$, and $\mathrm{Hg}$ ), display stable Cooper pair condensation at low
}

and pair removal Cooper pairs are virtually poised in the ground state of the closed-shell system $A_{0}$, ready to condense [i.e., $W\left(A_{0}+2\right), W\left(A_{0}-2\right) \rightarrow 0$, see Fig. 3], inducing a (fluctuating) alignment of pairspins perpendicular to the gauge $(z$ axis) and thus to a domain wall and associated generalized rigidity (see Fig. 13).

The fluctuations of the different pairspins are not correlated, so that the average of the total spin $\left\langle S_{\perp}\right\rangle$ in the $x-y$ plane [cf. Eq. (A11)] vanishes. However, $\left\langle s_{z}(v)\right\rangle=1 / 2\left[\left\langle N_{\nu}\right\rangle-1\right]$, which is directly related to the average number of particles, deviates from the HF value $( \pm 1 / 2)$. A convenient way to depict the dynamics of pairspins in gauge space is by means of the precessional cone. This cone is aligned along the $z$-quantization axis (for occupied levels), or in the opposite direction (for empty levels), in keeping with the fact that $\left\langle S_{\perp}\right\rangle=0$. Its opening angle $\tilde{\theta}(v)$ can be obtained from the relation

$$
\cos (\tilde{\theta}(v))=\left\langle s_{z}(v)\right\rangle /|S|=\left\langle s_{z}(v)\right\rangle / \sqrt{3 / 4} .
$$

In the HF limit, $\left\langle s_{z}(v)\right\rangle= \pm 1 / 2$, so that $\tilde{\theta}_{v}=54.5^{\circ}$ (for holes) or $125.5^{\circ}$ (for particles). Under the influence of pairing fluctuations a given pairspin may jump from the upper to the lower cone, in keeping with the modification of the occupation factors. A measure of these fluctuations can be given by a modified effective opening angle, closer to $90^{\circ}$, obtained substituting the actual value of $\left\langle s_{z}(v)\right\rangle$ in Eq. (22). The difference between the resulting value of $\tilde{\theta}$ and the HF value is displayed in Fig. 5.

An alternative way to represent the effect of pair fluctuations is by reexpressing the changes in the number of particles in terms of effective occupation amplitudes, similar to those of BCS theory [cf. Eq. (21)]:

$$
\begin{aligned}
& \left(V^{\mathrm{eff}}(v)\right)^{2}=2 Y_{\mathrm{rem}}^{2}\left(j_{v}\right) / \Omega_{j_{v}} ; \\
& \left(U^{\mathrm{eff}}(v)\right)^{2}=1-\left(V^{\mathrm{eff}}(v)\right)^{2}, \quad \epsilon_{j_{v}}>0 \\
& \left(U^{\mathrm{eff}}(v)\right)^{2}=2 Y_{\mathrm{add}}^{2}\left(j_{v}\right) / \Omega_{j_{v}} ; \\
& \left(V^{\mathrm{eff}}(v)\right)^{2}=1-\left(U^{\mathrm{eff}}(v)\right)^{2}, \quad \epsilon_{j_{v}} \leqslant 0 .
\end{aligned}
$$

The quantity $U^{\text {eff }} V^{\text {eff }}$ plays, in the normal case, the role the product $U^{\prime} V^{\prime}$ plays in the description of the superfluid phase within the framework of BCS theory (see Fig. 4). It is of notice, however, that $U^{\text {eff }} V^{\text {eff }}$ has a dynamical origin, while $U^{\prime} V^{\prime}$ a static one. In other words, the quantities displayed in Figs. 4 and 5 reflect the fact that pairing fluctuations in closed-shell nuclei tend to reproduce the picture characteristic of superfluid nuclei, although the phase transition is hindered by the presence of a large shell gap.

Consequently, as soon as the single-particle field $H_{\mathrm{sp}}-\mathrm{a}$ field that acts on the nuclear pairspin along the gauge $(z)$ axis

temperatures, becoming superconductors, while good conductors, independent electron motion metals (e.g., $\mathrm{Au}, \mathrm{Ar}, \mathrm{Cu}$ ) do not. In the nuclear case, arguably, one of the best nuclear embodiments of Cooper's model is the $\left|\mathrm{gs}\left({ }^{11} \mathrm{Li}\right)\right\rangle$ state, pair addition mode of ${ }^{9} \mathrm{Li}$. The associated single-particle system $\left({ }^{10} \mathrm{Li}\right)$ is not only unbound, but, more revealing, single-particle mean-field levels are so strongly dressed by the coupling to the bosonic (vibrational) modes of the medium that a new magic number becomes operative in this case, namely $N=6$ instead of $N=8$ (parity inversion phenomenon) (see Ref. [10], Chap. 11, and Ref. [16] and references therein). 


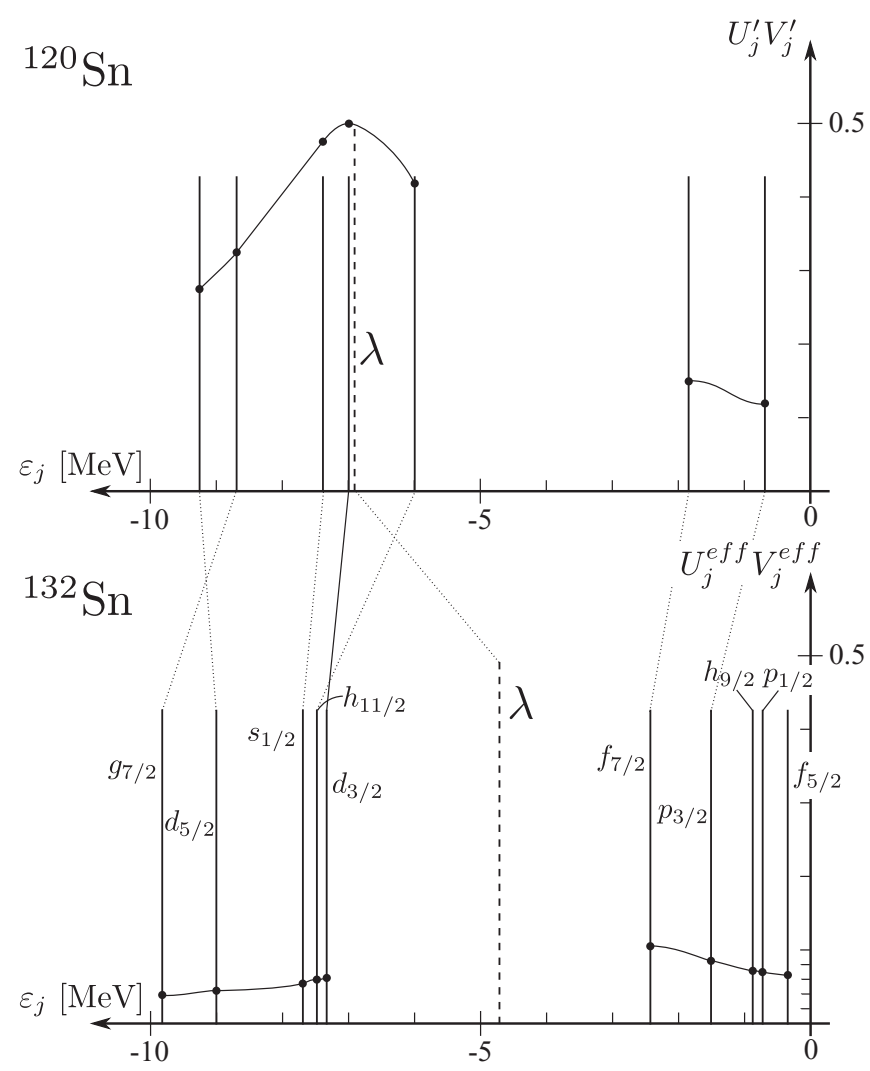

FIG. 4. (a) Value of the $U^{\prime} V^{\prime}$ products associated with ${ }^{120} \mathrm{Sn}$ (see Table II). (b) Value of the $U^{\text {eff }} V^{\text {eff }}$ products associated with the pair addition and removal modes of ${ }^{132} \mathrm{Sn}$, obtained using the values of the backwardsgoing amplitudes listed in Table III.

in a similar way in which a magnetic field acts in the case of a metallic superconductor [see Appendix A, discussion after Eq. (A27); see also discussion after Eq. (A35)]—is decreased, a fact that takes place moving away from magic numbers (in the case of, e.g., ${ }^{132} \mathrm{Sn}$ this implies reducing the single-particle gap $\left|\varepsilon_{j<}-\varepsilon_{j>}\right|$ from about $\approx 5 \mathrm{MeV}$ to few hundreds of $\mathrm{keV}$; see Tables II and III), nuclear Cooper pairs condense, the ground state of the corresponding nucleus becoming amenable to a BCS-type description, a fact that already takes place with the presence of two removal modes in $A_{0}$.

Of notice is the presence of strong fluctuations in pairspin observed in Fig. 5, as compared to the smooth variations shown in Fig. 1, idealization of the situation representative of a high-purity, metallic crystal (within this context see Fig. 13 of Appendix A). In fact, finite nuclei display orbitals that contribute very differently to pairing correlations, in particular hot orbitals (see, e.g., Refs. $[12,17,18]$ ), related to the different pair degeneracy $\Omega_{v}$ and with the relative amount of $s$ component of the different $j_{v}^{2}(0)$ pure twoparticle configurations. One can view such an imperfect pairspin alignment as a limitation of its applicability to FMB systems. Conversely, one can interpret it as a reflection of the richness with which these systems in general, and finite nuclei in particular, embody symmetry-breaking phase transitions, namely, among other things, in terms of very nonconventional normal phases, which display (virtually) traces of, e.g.,

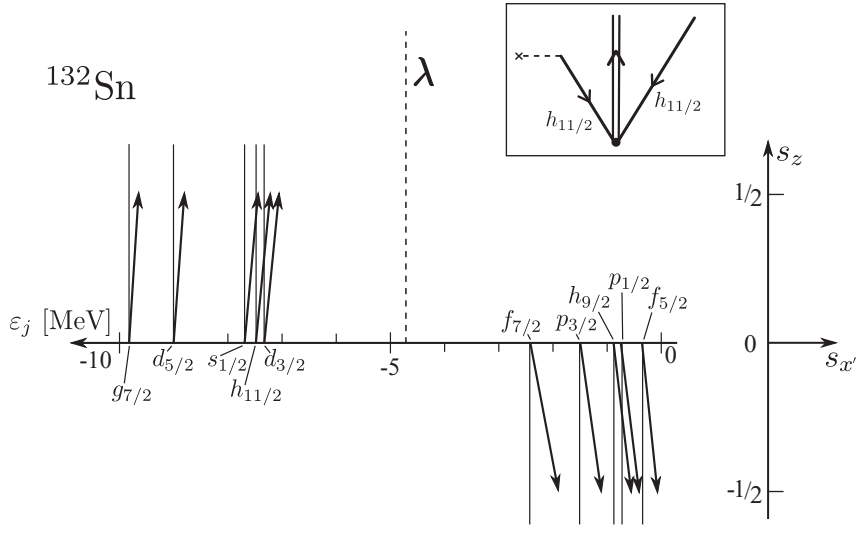

FIG. 5. Representation, in terms of pairspin alignment, of the dynamical ODLRO induced in the ground state of the doubly magic nucleus ${ }^{132} \mathrm{Sn}$, by the zero-point fluctuations (ground-state correlations) associated with pair addition and pair removal of the closed-shell system (for comparison with the superfluid system ${ }^{120} \mathrm{Sn}$ see Fig. 13 of Appendix A). The orientation of the arrows in this figure shows the difference $\tilde{\theta}_{v} \pm 54.5^{\circ}$ (for levels above or below the Fermi energy), multiplied by a factor 10 , to make it easily visible (see text). Within the present scenario, it is expected that one-particle transfer reactions on ${ }^{132} \mathrm{Sn}$ may, e.g., excite the $h_{11 / 2}^{-1} \otimes \mathrm{gs}\left({ }^{134} \mathrm{Sn}\right) 2 \mathrm{p}-1 \mathrm{~h}$ state of ${ }^{133} \mathrm{Sn}$ (see inset) with a weak, but likely observable cross section.

domain walls with varied degree of stability, dynamically violating the symmetry in question (gauge symmetry in the present case). These properties are precisely those that render the study of pairing correlations in nuclei central in the quest for the mechanism which are at the basis of the stability of nuclear species, in particular along the drip lines. ${ }^{2}$

In keeping with the fact that the $P^{\dagger}$ and $P$ are the basic operators entering both the pairing interaction $\left(H_{p}=-G P^{\prime \dagger} P^{\prime}\right)$ and the pair mean field $\left[-G \alpha_{0}^{\prime}\left(P^{\dagger}+P\right)+G\left(\alpha_{0}^{\prime}\right)^{2}\right]$, twonucleon transfer can be viewed as the specific probe of pairing correlations in nuclei, in a similar way as Coulomb excitation, inelastic scattering, and $\gamma$ decay are specific tools to probe (quadrupole) surface vibrations and rotations (see Fig. 2). In other words, specific information on $\alpha_{0}^{\prime}$ and $\alpha_{\text {dyn }}$ can be obtained through two-nucleon transfer reactions.

Because the correlation length associated with the nuclear Cooper pairs is

$$
\xi= \begin{cases}\frac{\hbar v_{F}}{2 \Delta^{\prime}}, & \left(\alpha_{0}^{\prime} \neq 0\right), \\ \frac{\hbar v_{F}}{2 E_{\text {corr }}}, & \left(\alpha_{0}^{\prime}=0\right),\end{cases}
$$

\footnotetext{
${ }^{2}$ Within this context one can mention the fact that if, instead of pairspins with two projections, one studies the properties of finite systems that depend on the alignment of a pairspin with 20 components, such as protein evolution and structure in which each projection corresponds to one of the 20 natually occurring amino acids, the fluctuations of pairspin and thus of emergent properties become even richer and subtler than in the case shown in Fig. 5 (see, e.g., Ref. [19] and references therein). From a technical point of view, but not only, the situation may be analogous to that resulting from tinkering with the Ising model to confront oneself with the Potts model.
} 
Cooper pair partners are correlated over distances considerably larger than nuclear dimensions $(\xi \approx 20-30 \mathrm{fm}$, as compared to $R \approx 5 \mathrm{fm}$ ). Consequently, from a nuclear structure point of view, Cooper pair transfer involves also regions in which the pairing interaction $G(x), x$ representing, e.g., the surfacesurface distance between target and projectile (see, e.g., Ref. [20], Chap. III), vanishes, a situation already known in condensed matter in connection with the Josephson effect [21-23]. This result, together with the fact that the depth of the single-particle potential $\left|V_{0}\right|(\approx 50 \mathrm{MeV})$ is much larger than $G(\approx 25 / A \mathrm{MeV})$, implies that, with an exception made for $Q$-value effects, successive transfer induced by the mean-field single-particle (Saxon-Woods-like) potential, is expected to be, as a rule, the largest contribution to the two-nucleon transfer cross sections [24-35]..$^{3}$ The difficulties to absorb this simple result by nuclear structure practitioners partially stems from the fact that, neglecting reaction details, the two-particle transfer cross sections gs $\rightarrow$ gs can be schematically written as

$$
\sigma(\mathrm{gs} \rightarrow \mathrm{gs}) \sim \begin{cases}\left|\alpha_{0}^{\prime}\right|^{2} & \left(\alpha_{0}^{\prime} \neq 0\right), \\ \left|\alpha_{\mathrm{dyn}}\right|^{2} & \left(\alpha_{0}^{\prime}=0\right)\end{cases}
$$

corresponding to the square modules of matrix elements of $P^{\dagger}$ and $P$, the associated spectroscopic amplitudes in the intrinsic coordinate system in gauge space $\mathcal{K}^{\prime}$ being (see, e.g., Ref. [12] and Appendix B)

$$
B(\nu \bar{v} ; J=0) \sim \mathcal{K}^{\prime}\left\langle\operatorname{BCS}(A+2)\left|P_{\nu}^{\prime \dagger}\right| \operatorname{BCS}(A)\right\rangle_{\mathcal{K}^{\prime}},
$$

where

$$
\begin{aligned}
& \mathcal{K}^{\prime}\left\langle\operatorname{BCS}(A+2)\left|P_{v}^{\prime \dagger}\right| \operatorname{BCS}(A)\right\rangle_{\mathcal{K}^{\prime}} \\
& \quad=U_{v}^{\prime}(A) V_{v}^{\prime}(A+2)={ }_{\mathcal{K}}^{\prime}\left\langle\operatorname{BCS}(A)\left|P_{v}^{\prime}\right| \operatorname{BCS}(A+2)\right\rangle_{\mathcal{K}^{\prime}} .
\end{aligned}
$$

\footnotetext{
${ }^{3}$ Within this context it is of notice that the incoming proton (distorted) wave, in, e.g., a ${ }^{A+2} \operatorname{Sn}(p, t)$ reaction, is diffused by the scattering center, i.e., by the ${ }^{A+2} \mathrm{Sn}$ target, into emergent distorted waves, in particular the one corresponding to the relative motion of a deuteron and of the ${ }^{A+1} \mathrm{Sn}$ system after the interaction $v_{\text {np }}$ has acted for the first time. Even when these two systems are at relative distances $r=\left|\vec{r}_{A+1} \mathrm{Sn}-\vec{r}_{d}\right|$ much larger than the target radius, the Cooper pair wave function describing the pair correlation of the picked-up neutron and of its partner in the ${ }^{A+1} \mathrm{Sn}$ system, has a finite probability amplitude centered on the outgoing deuteron, and this is so not only in the case of superfluid nuclei (such as, e.g., ${ }^{120} \mathrm{Sn}$ ) but also that of normal nuclei (such as, e.g., ${ }^{132} \mathrm{Sn}$ ). This is in keeping with the fact that the stability, collectivity, and associated correlation length associated with superfluid Cooper pairs and with pair addition and removal Cooper pairs are very similar, as discussed above. Making use of this finite amplitude, the interaction $v_{n p}$ acting a second time [see Eq. (38b) below] can trigger the ${ }^{A+1} \mathrm{Sn}$ Cooper pair partner to move into the deuteron leading to the triton and completing in this way the successive transfer process. From this narrative, it is not surprising that the paper in which the probabilistic interpretation of Schrödinger wave function was forcefully proposed, written by Born, describes a collisional process [36].
}

Now, thinking in terms of the transfer of one nucleon at a time, e.g., in the case of the reaction

$$
(A+2)+p \rightarrow F(\equiv A+1)+d \rightarrow A+t,
$$

one can write, in connection with the first step $[(A+2)+p \rightarrow$ $F+d]$,

$$
a_{v_{d}}^{\prime \dagger} a^{\prime}{ }_{v}|\operatorname{BCS}(A+2)\rangle|p\rangle \sim V_{v}^{\prime}(A+2) \alpha_{\bar{\nu}}^{\dagger}|\operatorname{BCS}(F)\rangle|d\rangle,
$$

and

$$
a_{v_{t}}^{\prime \dagger} a^{\prime}{ }_{\bar{\nu}}|\operatorname{BCS}(F)\rangle|d\rangle \sim U_{v}^{\prime}(A) V_{v}^{\prime}(A+2)|\operatorname{BCS}(F)\rangle|d\rangle,
$$

in connection with the second step $(F+d \rightarrow A+t)$, in keeping with the fact that

$$
\begin{aligned}
& a_{\nu}^{\prime \dagger}=U_{\nu}^{\prime} \alpha_{\nu}^{\dagger}+V_{\nu} \alpha_{\bar{\nu}}, \\
& a^{\prime}{ }_{\nu}=U_{\nu}^{\prime} \alpha_{\nu}+V_{\nu}^{\prime} \alpha_{\bar{\nu}}^{\dagger}, \\
& a_{\bar{\nu}}^{\prime \dagger}=U_{\nu}^{\prime} \alpha_{\bar{\nu}}^{\dagger}-V_{\nu}^{\prime} \alpha_{\nu}, \\
& a_{\bar{\nu}}^{\prime}=U_{\nu}^{\prime} \alpha_{\bar{\nu}}-V_{\nu}^{\prime} \alpha_{\nu}^{\dagger},
\end{aligned}
$$

and that $|\mathrm{BCS}\rangle$ is the quasiparticle vacuum. The primed quantities are referred to the intrinsic, body-fixed frame (see Appendixes A and B).

Consequently, the associated (successive) two-nucleon transfer spectroscopic amplitude,

$$
B\left(v^{2}(0)\right)=U_{v}^{\prime}(A) V_{v}^{\prime}(A+2),
$$

has the same dependence on the BCS occupation numbers as that displayed by the amplitudes associated with simultaneous transfer (order parameter) [12] and with the amplitude entering in the Cooper pair wave function. This last result is closely related to the smooth behavior of the BCS occupation parameters with mass number (see Appendix B). Summing up, pair coherence is maintained both in successive as well as in simultaneous transfer.

It is of notice that all the above results, which constitute the very essence of nuclear BCS, are not only inescapable, they are also almost tautological, at least for well bound nuclei. In fact, pairing in nuclei affects neither the single-particle energies $\varepsilon_{v}$ [see in any case Eq. (A27) and following discussion] nor the corresponding wave functions $\phi_{v}(\vec{r})$, but only the single-particle occupation probabilities. This takes place in a small $\left(\Delta^{\prime} / \varepsilon_{F} \approx 5 \times 10^{-2}\right)$ region around the Fermi energy. In this region, and in keeping with the structure of the BCS wave function, which takes into account the variety of excitations of pairs of particles $(\nu, \bar{v})$, so as to produce the most efficient mixing of empty and occupied states leading to Cooper pairs, the only possible excitation mechanism of the nuclear superfluid is that of breaking a Cooper pair, individual two-particle $(\nu \bar{v})$ excitations being already taken into account in the BCS ground state. It is then not surprising that the amplitudes of the Cooper pair wave function are $c_{v} \sim U_{v}^{\prime} V_{v}^{\prime}$ or that the absolute cross section for Cooper pair transfer is proportional to $\left(\sum_{v>0} U_{v}^{\prime} V_{v}^{\prime}\right)^{2}$. Least surprising is that these amplitudes and two-nucleon transfer processes involve not only $(n l j m, n l j-m)$ configurations, but also $\left(n l j m, n^{\prime} l j-\right.$ $m)$ as well as $\left[\left(J(A)+n_{1} l_{1} j_{1}\right)_{J_{1}} \otimes\left(J^{\prime}(a)+n_{2} l_{2} j_{2}\right)_{J_{1}}\right]_{0}$. This is in keeping with the nonorthogonality of the single-particle wave functions describing the target and projectile in the 

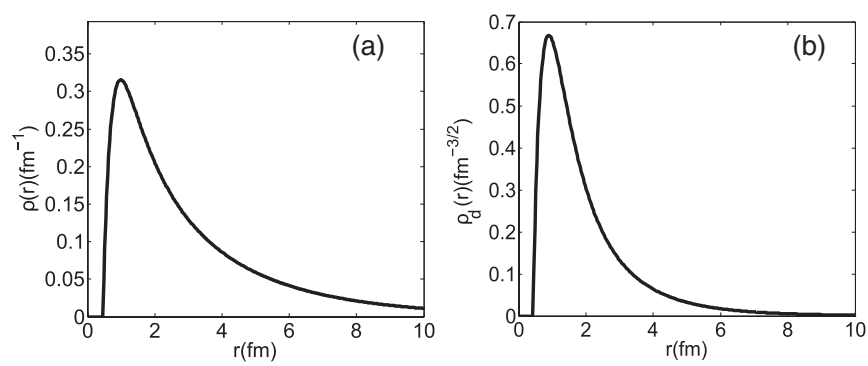

FIG. 6. (a) Radial function $\rho(r)$ (hard core $0.45 \mathrm{fm}$ ) entering the triton wave function. (b) Radial function $\rho_{d}(r)$ entering the deuteron wave function.

different channels $(a+A \rightarrow f+F \rightarrow b+B)$. Within this context, $n$ and $n^{\prime}$ are possible not only owing to the fact that partners of a Cooper pair feel different mean fields $\left(\phi_{n^{\prime} l j m}^{f}, \phi_{n l j m}^{F}\right)$, but also because a general nuclear structure treatment of pairing will include Cooper-like correlations associated with multipole pairing (see, e.g., Ref. [10], Sec. 5.3, and references therein), correlations which, in the present case, have not a dynamical origin (one works with $H_{p}=-G P^{\dagger} P$ ), but only a trivial kinematical one.

\section{REACTION MECHANISM}

In what follows we present the elements which enter the calculation of the absolute two-particle transfer differential cross section in terms of the reaction

$$
A+t \rightarrow B(\equiv A+2)+p,
$$

in which $A+2$ and $A$ denote the mass number of even nuclei in their ground state. In other words, one concentrates on $L=0$ transfer. The wave function of nucleus $A+2$ is written as

$$
\begin{aligned}
& \Psi_{A+2}\left(\xi_{A}, \mathbf{r}_{A 1}, \sigma_{1}, \mathbf{r}_{A 2}, \sigma_{2}\right) \\
& \quad=\psi_{A}\left(\xi_{A}\right) \sum_{l_{i}, j_{i}}\left[\phi_{l_{i}, j_{i}}^{A+2}\left(\mathbf{r}_{A 1}, \sigma_{1}, \mathbf{r}_{A 2}, \sigma_{2}\right)\right]_{0}^{0},
\end{aligned}
$$

the product of the wave function describing the ground state of the nucleus $A$, the corresponding relative (intrinsic) $3 A-3$ radial coordinates being denoted $\xi_{A}$, and of the wave function of two-correlated nucleons,

$$
\begin{aligned}
& {\left[\phi_{l_{i}, j_{i}}^{A+2}\left(\mathbf{r}_{A 1}, \sigma_{1}, \mathbf{r}_{A 2}, \sigma_{2}\right)\right]_{0}^{0}} \\
& \quad=\sum_{n m} a_{n m}\left[\varphi_{n, l_{i}, j_{i}}^{A+2}\left(\mathbf{r}_{A 1}, \sigma_{1}\right) \varphi_{m, l_{i}, j_{i}}^{A+2}\left(\mathbf{r}_{A 2}, \sigma_{2}\right)\right]_{0}^{0},
\end{aligned}
$$

the wave functions $\varphi_{n, l_{i}, j_{i}}^{A+2}(\mathbf{r}, \sigma)$ describing the single-particle motion of a nucleon in a mean-field potential, e.g., a Saxon-Woods potential. The two-neutron wave function in the triton can be written as $\phi_{t}\left(r_{p 1}, \sigma_{1}, r_{p 2}, \sigma_{2}\right)=$ $\rho\left(r_{p 1}\right) \rho\left(r_{p 2}\right) \rho\left(r_{12}\right)\left[\chi\left(\sigma_{1}\right) \chi\left(\sigma_{2}\right)\right]_{0}^{0}, r_{p 1}$ and $r_{p 2}$ denoting the modulus of the relative coordinate of each of the two neutrons involved in the transfer process, measured with respect to the proton, while $r_{12}$ denotes the modulus of the relative coordinate of the two neutrons in the triton. The deuteron wave function is written as $\phi_{d}\left(r_{p 1}, \sigma_{1}\right)=\rho_{d}\left(r_{p 1}\right) \chi\left(\sigma_{1}\right)$. The functions $\rho(r)$ and $\rho_{d}(r)$, as depicted in Fig. 6, are generated with the $p$ - $n$ Tang-Herndon interaction [37],

$$
\begin{aligned}
& v(r)=-v_{0} \exp \left(-k\left(r-r_{c}\right)\right), \quad r>r_{c}, \\
& v(r)=\infty \quad r<r_{c},
\end{aligned}
$$

where $k=2.5 \mathrm{fm}^{-1}$ and $r_{c}=0.45 \mathrm{fm}$ denotes the radius of the hard core. The depth $v_{0}$ is adjusted so as to reproduce the binding energy of the triton and of the deuteron, respectively. This hard-core potential is also used in the above expressions as the $n-p$ interaction potential responsible for neutron transfer.

The two-particle transfer differential cross section is written as

$$
\frac{d \sigma}{d \Omega}=\frac{\mu_{i} \mu_{f}}{\left(4 \pi \hbar^{2}\right)^{2}} \frac{k_{f}}{k_{i}}\left|T^{(1)}+T_{\mathrm{succ}}^{(2)}-T_{\mathrm{NO}}^{(2)}\right|^{2} .
$$

The amplitudes appearing in it describe the simultaneous,

$$
T^{(1)}=2 \sum_{l_{i}, j_{i}} \sum_{\sigma_{1} \sigma_{2}} \int d \mathbf{r}_{t A} d \mathbf{r}_{p 1} d \mathbf{r}_{A 2}\left[\phi_{l_{i}, j_{i}}^{A+2}\left(\mathbf{r}_{A 1}, \sigma_{1}, \mathbf{r}_{A 2}, \sigma_{2}\right)\right]_{0}^{0 *} \chi_{p B}^{(-) *}\left(\mathbf{r}_{p B}\right) v\left(r_{p 1}\right) \phi_{t}\left(r_{p 1}, \sigma_{1}, r_{p 2}, \sigma_{2}\right) \chi_{t A}^{(+)}\left(\mathbf{r}_{t A}\right),
$$

successive,

$$
\begin{aligned}
T_{\mathrm{succ}}^{(2)}= & 2 \sum_{l_{i}, j_{i}} \sum_{l_{f}, j_{f}, m_{f}} \sum_{\substack{\sigma_{1} \sigma_{2} \\
\sigma_{1}^{\prime} \sigma_{2}^{\prime}}} \int d \mathbf{r}_{d F} d \mathbf{r}_{p 1} d \mathbf{r}_{A 2}\left[\phi_{l_{i}, j_{i}}^{A+2}\left(\mathbf{r}_{A 1}, \sigma_{1}, \mathbf{r}_{A 2}, \sigma_{2}\right)\right]_{0}^{0 *} \chi_{p B}^{(-) * *}\left(\mathbf{r}_{p B}\right) v\left(r_{p 1}\right) \phi_{d}\left(r_{p 1}, \sigma_{1}\right) \varphi_{l_{f}, j_{f}, m_{f}}^{A+1}\left(\mathbf{r}_{\mathrm{A2}}, \sigma_{2}\right) \\
& \times \int d \mathbf{r}_{d F}^{\prime} d \mathbf{r}_{p 1}^{\prime} d \mathbf{r}_{A 2}^{\prime} G\left(\mathbf{r}_{d F}, \mathbf{r}_{d F}^{\prime}\right) \phi_{d}\left(r_{p 1}^{\prime}, \sigma_{1}^{\prime}\right)^{*} \varphi_{l_{f}, j_{f}, m_{f}}^{A+1 *}\left(\mathbf{r}_{\mathbf{A} \mathbf{2}}^{\prime}, \sigma_{2}^{\prime}\right) \frac{2 \mu_{d F}}{\hbar^{2}} v\left(r_{p 2}^{\prime}\right) \phi_{d}\left(r_{p 1}^{\prime}, \sigma_{1}^{\prime}\right) \phi_{d}\left(r_{p 2}^{\prime}, \sigma_{2}^{\prime}\right) \chi_{t A}^{(+)}\left(\mathbf{r}_{t A}^{\prime}\right),
\end{aligned}
$$

and nonorthogonal,

$$
\begin{aligned}
T_{\mathrm{NO}}^{(2)}= & 2 \sum_{l_{i}, j_{i}} \sum_{l_{f}, j_{f}, m_{f}} \sum_{\substack{\sigma_{1} \sigma_{2} \\
\sigma_{1}^{\prime} \sigma_{2}^{\prime}}} \int d \mathbf{r}_{d F} d \mathbf{r}_{p 1} d \mathbf{r}_{A 2}\left[\phi_{l_{i}, j_{i}}^{A+2}\left(\mathbf{r}_{A 1}, \sigma_{1}, \mathbf{r}_{A 2}, \sigma_{2}\right)\right]_{0}^{0 *} \chi_{p B}^{(-) *}\left(\mathbf{r}_{p B}\right) v\left(r_{p 1}\right) \phi_{d}\left(r_{p 1}, \sigma_{1}\right) \varphi_{l_{f}, j_{f}, m_{f}}^{A+1}\left(r_{A 2}, \sigma_{2}\right) \\
& \times \int d \mathbf{r}_{p 1}^{\prime} d \mathbf{r}_{A 2}^{\prime} d \mathbf{r}_{d F}^{\prime} \phi_{d}\left(r_{p 1}^{\prime}, \sigma_{1}^{\prime}\right)^{*} \varphi_{l_{f}, j_{f}, m_{f}}^{A+1 *}\left(\mathbf{r}_{\mathbf{A} 2}^{\prime}, \sigma_{2}^{\prime}\right) \phi_{d}\left(r_{p 1}^{\prime}, \sigma_{1}^{\prime}\right) \phi_{d}\left(r_{p 2}^{\prime}, \sigma_{2}^{\prime}\right) \chi_{t A}^{(+)}\left(\mathbf{r}_{t A}^{\prime}\right),
\end{aligned}
$$


contributions to the transfer process. In these expressions, $\varphi_{l_{f}, j_{f}, m_{f}}^{A+1}\left(\mathbf{r}_{\mathbf{A} \mathbf{2}}^{\prime}, \sigma_{2}^{\prime}\right)$ are the wave functions describing the intermediate states of the nucleus $F \equiv A+1$, generated as solutions of a Saxon-Woods potential. We have chosen the so-called post-post-representation [20], in which the $p-n$ interaction appears twice in the successive amplitude. The Green's function $G\left(\mathbf{r}_{d F}, \mathbf{r}_{d F}^{\prime}\right)$ propagates the intermediate channel $d, F$ and can be expanded in partial waves

$$
\begin{aligned}
G\left(\mathbf{r}_{d F}, \mathbf{r}_{d F}^{\prime}\right)= & i \sum_{l} \sqrt{2 l+1} \frac{f_{l}\left(k_{d F}, r_{<}\right) P_{l}\left(k_{d F}, r_{>}\right)}{k_{d F} r_{d F} r_{d F}^{\prime}} \\
& \times\left[Y^{l}\left(\hat{r}_{d F}\right) Y^{l}\left(\hat{r}_{d F}^{\prime}\right)\right]_{0}^{0} .
\end{aligned}
$$

The functions $f_{l}\left(k_{d F}, r\right)$ and $P_{l}\left(k_{d F}, r\right)$ are the regular and the irregular solutions of a Schrödinger equation associated with a suitable optical potential and an energy equal to the kinetic energy in the intermediate state. In most cases of interest, the result is hardly altered if one uses the same energy of relative motion for all the intermediate states. This representative energy is calculated when both nuclei appearing in the intermediate state are in their ground states. The validity of this approximation can break down in particular cases. For example, in the case in which some relevant intermediate states are strongly off shell, in which case their contribution is significantly quenched. An interesting situation can develop when this situation becomes operative for all possible intermediate states, in which case they can only be virtually populated, thus emphasizing the role of simultaneous transfer.

\section{THE ISOTOPIC CHAIN ${ }_{50}^{100} \mathrm{Sn}_{50}{ }^{-132}{ }_{50} \mathrm{Sn}_{82}$}

A collective mode is characterized by (1) an enhanced cross section or transition probability and (2) a simple expression of its energy as a function of the quantum number characterizing the states connected by the transition. This quantum number is related to restoration of the symmetry violation (static or dynamical, e.g., particle number in the case of pairing rotations and vibrations, angular momentum in the case of, e.g., quadrupole rotations and vibrations).

For example, in the case of a quadrupole rotational band of a 3D-deformed nucleus such as, e.g., ${ }^{152} \mathrm{Dy},(1)$ corresponds to the $B(E 2)$ transition probability, measured, e.g., in the terms of single-particle Weisskopf units (of the order of $10^{3}$ in the example chosen), while (2) corresponds to $E_{I}=\left(\hbar^{2} / 2 \mathcal{J}\right) I(I+1)$, $I$ being the angular momentum of the $\operatorname{system}(I=0,2,4, \ldots)$. In the case of pairing rotational bands, (1) corresponds to the absolute value of the two-nucleon differential cross section, measured in terms of the average pure two-particle units [12, 17,18] (typical value of the enhancement factor being, in the case of $\mathrm{Sn}$ isotopes, of the order of $10^{2}$ ), while (2) corresponds to $E_{N}=\left(\hbar^{2} / 2 \mathcal{I}\right)\left(N-N_{0}\right)^{2}, N$ being the number of particles associated with the condensate, namely with the neutrons in the case of ${ }_{50}^{A} \mathrm{Sn}_{N}$, while $N_{0}$ is the mean number of neutrons representative of the particle number wave packet describing the superfluid $\mathrm{Sn}$ isotopes $\left(N_{0} \approx 68\right.$, see discussion below). Of these two quantities, (1) is arguably the most representative one. This is because accidental degeneracies or residual interactions may modify the energies without much altering the long-range correlation of the coherent state. ${ }^{4}$ This is also the reason why, in what follows, use is made of the single $j$-shell model to discuss the basic features of the pairing rotational modes.

In this section we present evidence of the accuracy with which the model of pairing rotations discussed in Sec. II, together with the two-nucleon transfer reaction scheme summarized in the last section, allows for an overall quantitative description of the absolute value of the two-nucleon transfer cross sections, when use is made of global optical parameters to describe the (three) elastic channels involved in the process. Consequently, the predictions given in Sec. IV B concerning the pairing vibrational spectrum expected in connection with the two unstable closed-shell systems ${ }^{132} \mathrm{Sn}$ and the most exotic one ${ }^{100} \mathrm{Sn}$ can be considered potentially important and likely quantitative.

\section{A. Pairing rotations}

In Fig. 7 we display the value of the absolute differential cross sections associated with the reactions ${ }_{50}^{A+2} \mathrm{Sn}(p, t){ }_{50}^{A} \mathrm{Sn}(\mathrm{gs})$ for which absolute measurements have been reported in the literature, in comparison with the experimental data [38-44] (see also Refs. [45,46]). The corresponding integrated cross sections are collected in Table IV. In all cases the contribution of the successive process is the dominant one. Examples of two-nucleon spectroscopic amplitudes obtained from BCS calculations are displayed in Table II $\left[U, V\right.$ for $\left.{ }^{120} \mathrm{Sn}(p, t){ }^{118} \mathrm{Sn}\right]$. They have been computed solving the gap and number equations with a monopole interaction acting on the bound orbitals, calculated as the eigenfunctions of a standard parametrized Saxon-Woods potential, and imposing that the gap reproduces the value obtained from the empirical odd-even mass differences for the various isotopes. The BCS spectroscopic amplitudes are in good agreement with those predicted by extended shell-model calculation (see Refs. [42,43] and references therein). The optical parameters in the entrance, intermediate, and final channel were taken from references in Refs. [38-43] and from Ref. [47] for the deuteron channel.

From the above results one can posit that theory provides an account of the experimental absolute differential cross section well within the experimental errors and, arguably, without free parameters.

Let us now concentrate our attention on the value and on the structure of the probability amplitude for two nucleons, at $\vec{r}$ and $\vec{r}^{\prime}$, to belong to a Cooper pair, namely $\alpha_{0}^{\prime}\left(\vec{r}, \vec{r}^{\prime}\right)=$ $\sum_{\nu>0} c_{\nu} \phi_{v}(\vec{r}) \phi_{\bar{\nu}}\left(\overrightarrow{r^{\prime}}\right)$ [see Eqs. (16) and (31)]. That is, the nuclear structure component of the two-particle transfer crosssection amplitude. To clarify the physics at the basis of the BCS description of pairing rotational bands, we discuss two scenarios for the case of the ${ }^{120} \mathrm{Sn}(p, t){ }^{118} \mathrm{Sn}$ reaction. In the first one, we consider all bound single-particle states, the cutoff energy being $E_{\text {cutoff }}=0 \mathrm{MeV}$. In the second case one sets

\footnotetext{
${ }^{4}$ Within this context one can mention the fact that, was it not for $H_{\mathrm{sp}}$, all pairspins would line up in the $(x, y)$ plane transverse to the gauge axis $z$ (see Appendix A), the pairspin alignment picture essentially becoming "exact" under such condition.
} 

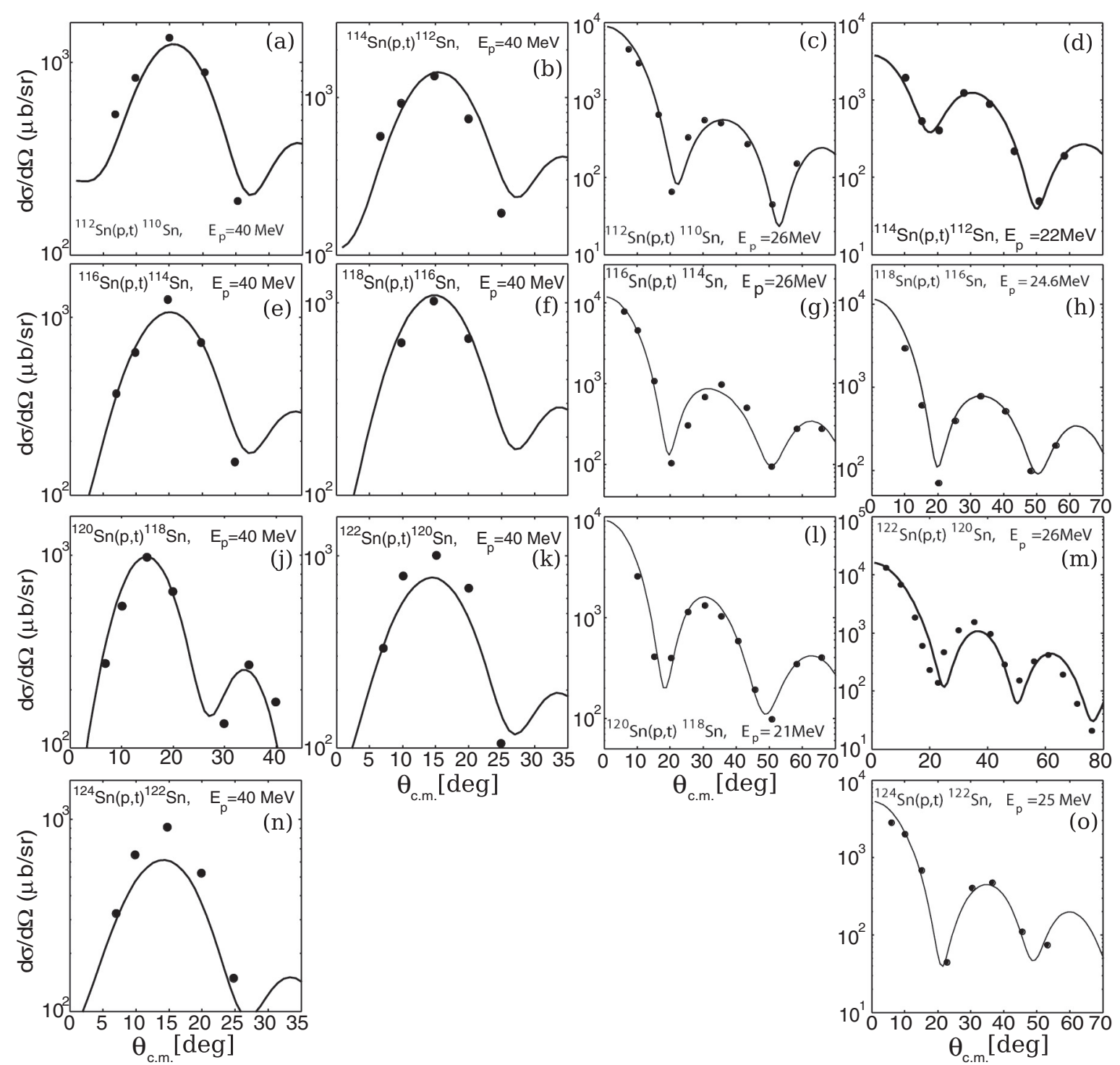

$\theta_{\text {c.m. }}[\mathrm{deg}]$
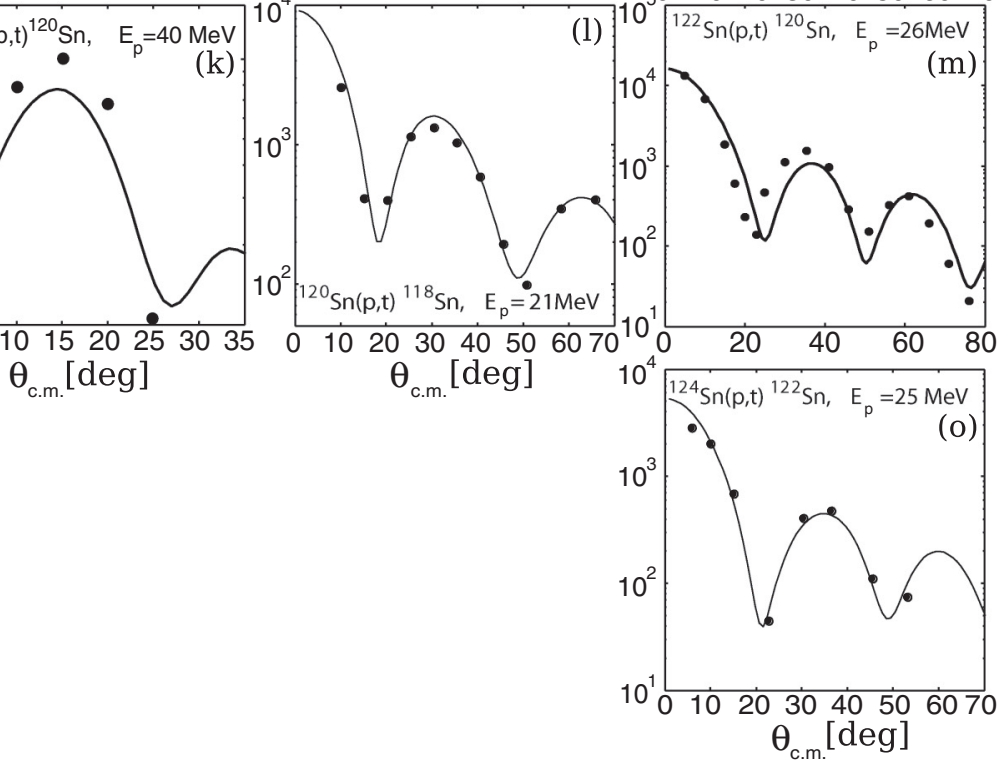

FIG. 7. Predicted absolute differential ${ }^{A+2} \operatorname{Sn}(p, t){ }^{A} \mathrm{Sn}(\mathrm{gs})$ cross sections for bombarding energies $E_{p}=40 \mathrm{MeV}$ (in the two left columns) and $21 \mathrm{MeV} \leqslant E_{p} \leqslant 26 \mathrm{MeV}$ (in the two right columns) in comparison with the experimental data (see Refs. [38-44], respectively).

$E_{\text {cutoff }}=60 \mathrm{MeV}$, discretizing the continuum inside a spherical box of $15 \mathrm{fm}$ of radius. The BCS gap $\left(\Delta^{\prime}=1.47 \mathrm{MeV}\right.$; experimental value) and number $(N=70)$ equations lead to $G=0.18 \mathrm{MeV}$ and $\lambda=-6.72 \mathrm{MeV}$ in the first case and $G=0.05 \mathrm{MeV}$ and $\lambda=-6.9 \mathrm{MeV}$ in the second one. The associated Cooper pair probability distributions in $r$ space are essentially identical (see Fig. 8 and Tables I and II). It is then not surprising that they lead to essentially the same absolute value of the two-particle transfer cross section associated with the reaction ${ }^{120} \mathrm{Sn}(p, t){ }^{118} \mathrm{Sn}(\mathrm{gs})$.

Let us now repeat the argument, but this time in terms of $\sigma(\mathrm{gs} \rightarrow \mathrm{gs}) \sim\left(\Delta^{\prime} / G\right)^{2}$, as it customarily done since the first publication that introduced it [48]. Because the pairing gap has been fixed to reproduce the experimental value $(1.47 \mathrm{MeV})$, one obtains in the case of $E_{\text {cutoff }}=0 \mathrm{MeV}\left(\Delta^{\prime} / G\right)^{2} \sim 70$ and $\left(\Delta^{\prime} / G\right)^{2} \sim 889$ in the case of $E_{\text {cutoff }}=60 \mathrm{MeV}$. This result emphasizes the problem of working with an expression which contains explicitly the pairing coupling constant.
One could argue that such an objection could also be leveled against the relation $\sigma \sim\left|\alpha_{0}\right|^{2}$. Note, however, that a $(p, t)$ reaction would hardly feel the effect of contributions far removed from the Fermi surface $\lambda$. This is in keeping with the fact that transfer to levels lying far away from $\lambda$ will be unfavorable owing to $Q$-value effects. If one argues in terms of the relative distance $r$ between target and projectile ( $r \gg R_{0}$ for continuumlike contributions; $r<R_{0}$ for deeply boundlike contributions), the outcome is similar. In fact, for large distances the two-particle transfer form factor vanishes while at small distances the outgoing tritium will experience strong absorption (see Appendix D).

In fact, considering only the contribution to $\alpha_{0}^{\prime}$ arising from the valence orbitals, that is, essentially those contributing to the "naked" vision of the Cooper pair wave functions, one obtains $\alpha_{0}^{\prime}=2.12$ and $\alpha_{0}^{\prime}=2.08$, respectively, and thus, a negligible squared relative difference between the two predicted cross sections, namely $(0.04 / 2.1)^{2} \approx 2 \times 10^{-3}$. 
TABLE IV. Absolute cross section associated with the ${ }^{A+2} \operatorname{Sn}(p, t)^{A} \operatorname{Sn}(g s)$ cross sections (i.e., between the members of the Sn-ground state pairing rotational band) calculated as described in the text, in comparison with the experimental findings.

\begin{tabular}{|c|c|c|c|}
\hline \multirow[b]{3}{*}{${ }^{112} \mathrm{Sn}(p, t){ }^{110} \mathrm{Sn}, E_{p}=26 \mathrm{MeV}$} & \multicolumn{3}{|c|}{$\sigma(\mathrm{gs} \rightarrow \mathrm{gs})$} \\
\hline & \multirow{2}{*}{$\begin{array}{c}\text { Theory } \\
1310^{\mathrm{a}}\end{array}$} & \multicolumn{2}{|c|}{ Experiment $^{\mathrm{c}, \mathrm{d}}$} \\
\hline & & $1309 \pm 200( \pm 14)^{\mathrm{a}}$ & {$\left[6^{\circ} \leqslant \theta \leqslant 62.7^{\circ}\right]$} \\
\hline${ }^{114} \mathrm{Sn}(p, t){ }^{112} \mathrm{Sn}, E_{p}=22 \mathrm{MeV}$ & $1508^{\mathrm{a}}$ & $1519.3 \pm 228( \pm 16.2)^{\mathrm{a}}$ & {$\left[7.64^{\circ} \leqslant \theta \leqslant 62.24^{\circ}\right]$} \\
\hline${ }^{116} \mathrm{Sn}(p, t){ }^{114} \mathrm{Sn}, E_{p}=26 \mathrm{MeV}$ & $2267^{\mathrm{a}}$ & $2492 \pm 374( \pm 32)^{\mathrm{a}}$ & {$\left[4^{\circ} \leqslant \theta \leqslant 70^{\circ}\right]$} \\
\hline${ }^{118} \mathrm{Sn}(p, t){ }^{116} \mathrm{Sn}, E_{p}=24.6 \mathrm{MeV}$ & $1460^{\mathrm{a}}$ & $1345 \pm 202( \pm 24)^{\mathrm{a}}$ & {$\left[7.63^{\circ} \leqslant \theta \leqslant 59.6^{\circ}\right]$} \\
\hline${ }^{120} \mathrm{Sn}(p, t)^{118} \mathrm{Sn}, E_{p}=21 \mathrm{MeV}$ & $2440^{\mathrm{a}}$ & $2250 \pm 338( \pm 14)^{\mathrm{a}}$ & {$\left[7.6^{\circ} \leqslant \theta \leqslant 69.7^{\circ}\right]$} \\
\hline${ }^{122} \mathrm{Sn}(p, t){ }^{120} \mathrm{Sn}, E_{p}=26 \mathrm{MeV}$ & $2429^{\mathrm{a}}$ & $2505 \pm 376( \pm 18)^{\mathrm{a}}$ & {$\left[2.5^{\circ} \leqslant \theta \leqslant 78.5^{\circ}\right]$} \\
\hline${ }^{124} \mathrm{Sn}(p, t){ }^{122} \mathrm{Sn}, E_{p}=25 \mathrm{MeV}$ & $918^{\mathrm{a}}$ & $958 \pm 144( \pm 15)^{\mathrm{a}}$ & {$\left[4^{\circ} \leqslant \theta \leqslant 57^{\circ}\right]$} \\
\hline${ }^{112} \mathrm{Sn}(p, t)^{110} \mathrm{Sn}, E_{p}=40 \mathrm{MeV}$ & $3349^{b}$ & \multicolumn{2}{|c|}{$3715 \pm 1114^{\mathrm{b}}$} \\
\hline${ }^{114} \mathrm{Sn}(p, t){ }^{112} \mathrm{Sn}, E_{p}=40 \mathrm{MeV}$ & $3790^{\mathrm{b}}$ & \multicolumn{2}{|c|}{$3776 \pm 1132^{b}$} \\
\hline${ }^{116} \mathrm{Sn}(p, t){ }^{114} \mathrm{Sn}, E_{p}=40 \mathrm{MeV}$ & $3085^{\mathrm{b}}$ & \multicolumn{2}{|c|}{$3135 \pm 940^{\mathrm{b}}$} \\
\hline${ }^{118} \mathrm{Sn}(p, t){ }^{116} \mathrm{Sn}, E_{p}=40 \mathrm{MeV}$ & $2563^{\mathrm{b}}$ & \multicolumn{2}{|c|}{$2294 \pm 668^{b}$} \\
\hline${ }^{120} \mathrm{Sn}(p, t){ }^{118} \mathrm{Sn}, E_{p}=40 \mathrm{MeV}$ & $3224^{b}$ & \multicolumn{2}{|c|}{$3024 \pm 907^{\mathrm{b}}$} \\
\hline${ }^{122} \mathrm{Sn}(p, t){ }^{120} \mathrm{Sn}, E_{p}=40 \mathrm{MeV}$ & $2339^{\mathrm{b}}$ & \multicolumn{2}{|c|}{$2907 \pm 872^{b}$} \\
\hline${ }^{124} \mathrm{Sn}(p, t){ }^{122} \mathrm{Sn}, E_{p}=40 \mathrm{MeV}$ & $1954^{\mathrm{b}}$ & \multicolumn{2}{|c|}{$2558 \pm 767^{b}$} \\
\hline
\end{tabular}

$\overline{\mathrm{a}} \mu \mathrm{b}$; the number in parentheses corresponds to the statistical errors; the numbers in square brackets provide the angular range of integration of the absolute two-particle differential cross sections.

${ }^{\mathrm{b}} \mu \mathrm{b} / \mathrm{sr}\left[\sum_{i=1}^{N}(d \sigma / d \Omega)\right.$; differential cross section summed over the few, $N=3-7$ experimental points].

${ }^{\mathrm{c}}$ References [38-43].

${ }^{\mathrm{d}}$ Reference [44].

Summing up, because the pair condensed state can be viewed as a coherent state which behaves essentially classically when viewed in terms of its building block (Cooper pair) the description of pairing rotational bands provided by the BCS model in terms of a coupling constant and an energy cutoff can be considered essentially "exact" when probed with two-nucleon transfer processes, reactions which filter the inaccuracies of each individual $U_{v} V_{v}$ component, emphasizing the off-diagonal long-range order provided by the phase coherence (cf. Ref. [49]). In fact, studying nuclear Cooper pair condensation in terms of, e.g., single-nucleon transfer (see, e.g., Refs. [50,51], the individual inaccuracies of the BCS occupation numbers cannot be averaged out. As a consequence, the overall agreement between theory and experiment is much poorer than that reflected by, e.g., the results collected in Table IV. The above arguments provide further evidence of why two-nucleon transfer is the specific probe of pairing superfluidity.

In Fig. 9 a quantity closely related to the $\mathrm{Sn}$ isotopes binding energy is reported as a function of the number of neutrons. Also displayed is the best parabolic fit to these energies, a quantity to be compared with

$$
E_{N}=\frac{\hbar^{2}}{2 \mathcal{I}}\left(N-N_{0}\right)^{2}
$$

namely the energy associated with the members of the pairing rotational band.

A simple estimate of the pairing rotational band moment of inertia is given by the single $j$-shell model [see, e.g., Ref. [10], Appendix $\left.\mathrm{H}, \hbar^{2} / 2 \mathcal{I}=G / 4 \approx 25 /\left(4 N_{0}\right) \mathrm{MeV}\right]$.
This estimate turns out to be rather accurate, even beyond expectation. Of notice is that, to the extent that one is discussing properties of a coherent state such as that described by Eq. (12), for which $H_{\mathrm{sp}}$ plays a secondary role [see discussion following Eq. (A27) in Appendix A] this is not a surprising result. As can be seen from Fig. 9, the estimate (40) is rather accurate except close to $N=50$ and $N=82$, in keeping with the fact that, as discussed before, the pairing deformed picture $\left(\alpha_{0}^{\prime} \neq 0\right)$ breaks down around the closed shell $\left(\alpha_{0}^{\prime}=0\right)$, where a vibrational regime [associated with the dynamic distortion $\alpha_{\text {dyn }}$, see Eq. (18)] is expected to be valid.

Also reported in Fig. 9 are the integrated values of the measured absolute two-particle transfer cross sections. Naively, one would expect a marked constancy of these transitions, in keeping with the fact that the (pairing) rotational model implies a common intrinsic (deformed) state [Cooper pair condensate; see Eq. (12)]. However, owing to the fact that the number of Cooper pairs contributing to the pairing distortion $\alpha_{0}^{\prime}$ is rather small (less than 10), one expects strong fluctuations in this quantity $\left(\Delta \alpha_{0}^{\prime} / \alpha_{0}^{\prime} \approx \sqrt{7} / 7 \approx 0.4\right)$ and consequently in the two-particle transfer cross section $\left(\sigma \sim \alpha_{0}^{\prime 2}\right.$, i.e., fluctuations in $\sigma$ of the order of $100 \%$ ).

In keeping with the analogy presented in Fig. 2, in the case of electromagnetic transition between members of a quadrupole rotational band one expects in heavy nuclei fluctuations of the order of $(\sqrt{250} / 250)^{2}$, i.e., less than $1 \%$. Within this context the average value of the absolute experimental cross sections in the energy range $E_{p}=$ $21-26 \mathrm{MeV}$ reported in Table IV is $1762 \mu \mathrm{b}$, while the average difference between experimental and predicted values is 

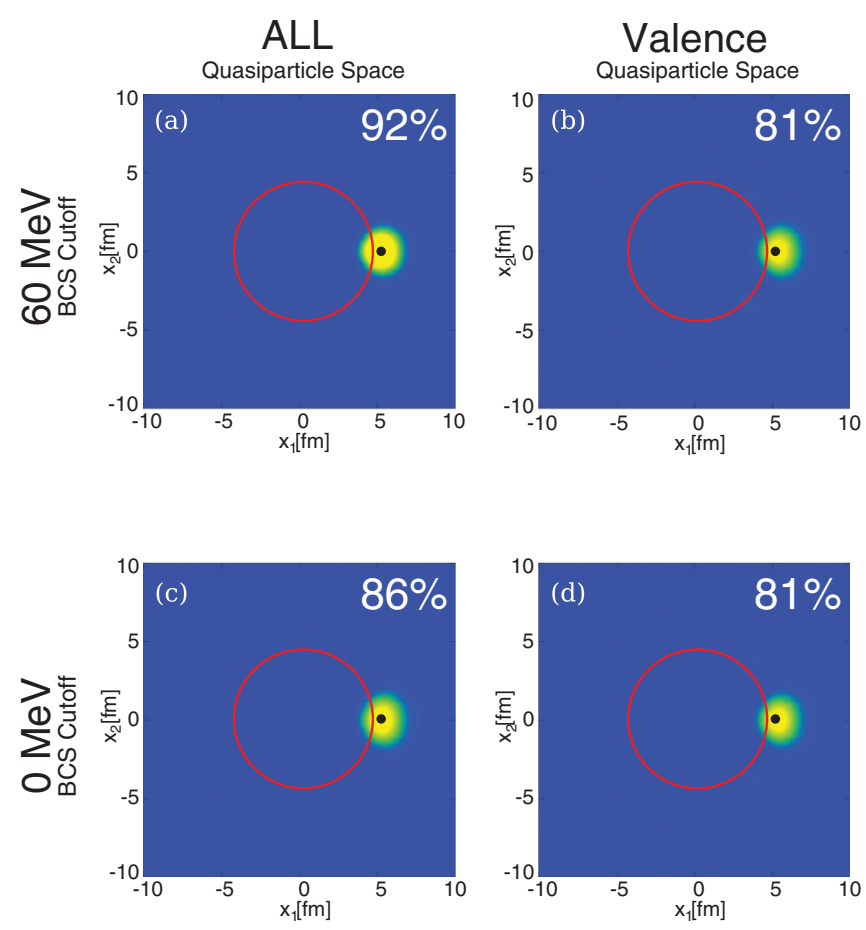

FIG. 8. (Color online) Spatial structure of a two-neutron Cooper pair of ${ }^{120} \mathrm{Sn}$ [see Eqs. (B16), (B17), and (B23)]. The modulus squared wave function $\left|\Psi_{0}\left(\vec{r}_{1}, \vec{r}_{2}\right)\right|^{2}=\left|\left\langle\tilde{0} \mid \vec{r}_{1}, \vec{r}_{2}\right\rangle\right|^{2}$ (see Tables I and II), multiplied by $16 \pi^{2} r_{1}^{2} r_{2}^{2}$ and normalized to unity, is displayed as a function of the Cartesian coordinates $x_{1}=r_{2} \cos \theta_{12}$ and $x_{2}=r_{2} \sin \theta_{12}$ of particle 2 , for a fixed value of $r_{1}=x_{1}=5 \mathrm{fm}$ (black dot) of particle 1 , close to the surface of the nucleus (red circle). The numerical percentages correspond to the two-nucleon integrated density in a spherical box of radius $4 \mathrm{fm}$ centered at the coordinates of the fixed particle.

$94 \mu \mathrm{b}$. Thus, the discrepancies between theory and experiment are bound in the interval $0 \leqslant \mid \sigma_{\exp }(i \rightarrow f)-\sigma_{\text {th }}(i \rightarrow$ $f) \mid / \sigma_{\exp }(i \rightarrow f) \leqslant 0.09$, the average discrepancy being $5 \%$.

In Fig. 10 the excited, pairing rotational band associated with the average value of the $0^{+}$pairing vibrational states with energy $\leqslant 3 \mathrm{MeV}$, is displayed together with the best parabolic fit. Also given is the relative $(p, t)$ integrated cross section normalized with respect to the gs $\rightarrow$ gs transitions, a value which is in all cases $\leqslant 8 \%$, in overall agreement with the single $j$-shell estimate (see Ref. [10], Appendix H), given in the inset to the figure. The result testifies to the weak cross talk between pairing rotational bands and thus of the robust off-diagonal, long-range order coherence of these modes.

\section{B. Pairing vibrational band in closed-shell nuclei}

The expected pairing vibrational spectrum (harmonic approximation, see Refs. [10-12] and references therein) associated with the closed-shell exotic nucleus ${ }^{132} \mathrm{Sn}[2,3]$, up to two-phonon states has been published in Fig. 3 of Ref. [45]. Within this approximation, the one-phonon states are the pair addition $|a\rangle=\left|\mathrm{gs}\left({ }^{134} \mathrm{Sn}\right)\right\rangle$ and pair removal $|r\rangle=$ $\left|\mathrm{gs}\left({ }^{130} \mathrm{Sn}\right)\right\rangle$ modes. The two-phonon $0^{+}\left(\left|p v\left({ }^{132} \mathrm{Sn}\right)\right\rangle=|r\rangle \otimes\right.$ $\left.|a\rangle=\left|0^{+}\left({ }^{132} \mathrm{Sn}\right) ; 6.5 \mathrm{MeV}\right\rangle\right)$ pairing vibrational $[(2 \mathrm{p}-2 \mathrm{~h})$-like] state of ${ }^{132} \mathrm{Sn}$, is predicted at an excitation energy of $6.5 \mathrm{MeV}$ (see Fig. 3). The absolute two-particle transfer differential

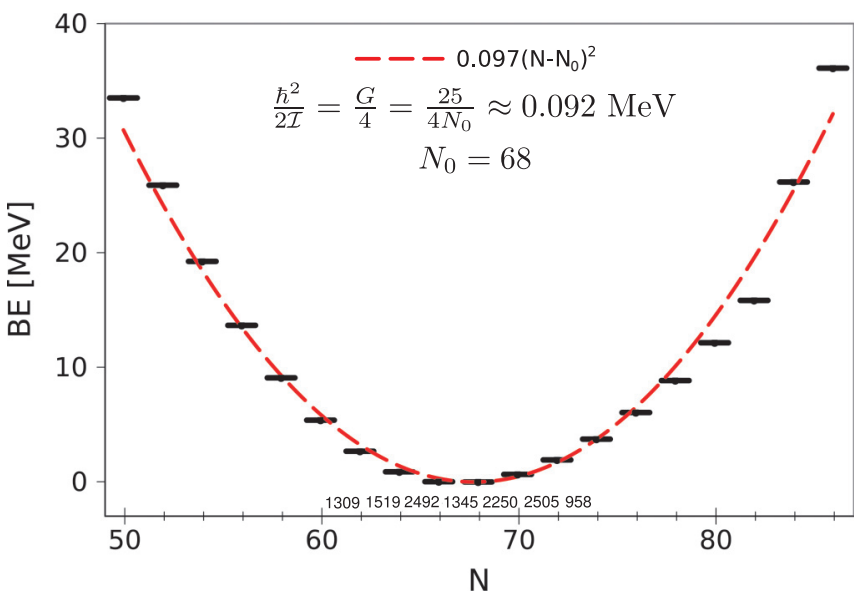

FIG. 9. (Color online) Pairing rotational band along the tin isotopes. The lines represent the energies calculated according to the expression $B E=B\left({ }^{50+N} \mathrm{Sn}_{N}\right)-8.124 N+46.33$ [10], subtracting the contribution of the single nucleon addition to the nuclear binding energy obtained by a linear fitting of the binding energies of the whole Sn chain. The estimate of $\hbar^{2} / 2 \mathcal{I}$ was obtained using the single $j$-shell model (see, e.g., Ref. [10], Appendix H).The numbers given on the abscissa are the absolute value of the experimental gs $\rightarrow$ gs cross section (in units of $\mu \mathrm{b}$; see Table IV).

cross sections associated with $|a\rangle$ and $|r\rangle$, namely,

$$
\begin{array}{ll}
{ }^{134} \mathrm{Sn}(p, t){ }^{132} \mathrm{Sn}(\mathrm{gs}), & \left(E_{\mathrm{CM}}=20 \mathrm{MeV}\right), \\
{ }^{132} \mathrm{Sn}(p, t){ }^{130} \mathrm{Sn}(\mathrm{gs}), & \left(E_{\mathrm{CM}}=26 \mathrm{MeV}\right),
\end{array}
$$

have been reported in the insets. Using detailed balance the reactions

$$
\begin{array}{ll}
{ }^{130} \mathrm{Sn}(t, p){ }^{132} \mathrm{Sn}\left(0^{+} ; 6.5 \mathrm{MeV}\right), & \left(E_{\mathrm{CM}}=20 \mathrm{MeV}\right), \\
{ }^{134} \mathrm{Sn}(p, t){ }^{132} \mathrm{Sn}\left(0^{+} ; 6.5 \mathrm{MeV}\right), & \left(E_{\mathrm{CM}}=26 \mathrm{MeV}\right),
\end{array}
$$

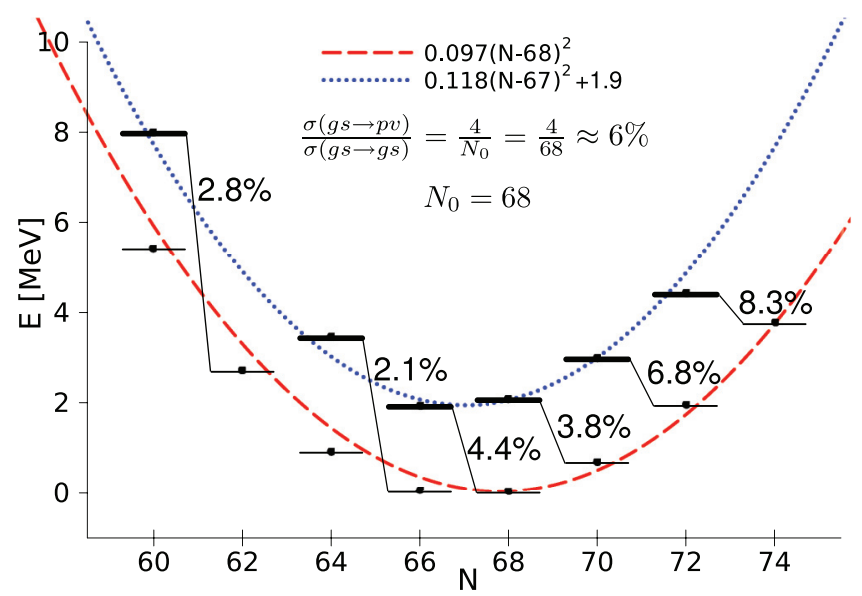

FIG. 10. (Color online) The weighted average energies $\left(E_{\text {exc }}=\right.$ $\left.\sum_{i} E_{i} \sigma_{i} / \sum_{i} \sigma_{i}\right)$ of the excited $0^{+}$states below $3 \mathrm{MeV}$ in the $\mathrm{Sn}$ isotopic chain are shown on top of the pairing rotational band, already displayed in Fig. 9. Also indicated is the percentage of cross section for two-neutron transfer to excited states, normalized to the cross sections populating the ground states. The estimate of the ratio of cross sections displayed on top of the figure was obtained making use of the single $j$-shell model (see, e.g., Ref. [10], Appendix H). 
are, within the harmonic approximation, equivalent to Eqs. (41) and (42), except for the relative flux which is determined by the ratio $k_{f} / k_{i}$.

Similar calculations to the ones discussed above have been carried out for the closed-shell nucleus ${ }^{100} \mathrm{Sn}$, the results again being collected in Fig. 3 of Ref. [45]. In this case the two-phonon $0^{+}$, pairing vibrational mode of ${ }^{100} \mathrm{Sn}$ is expected, again within the harmonic approximation, at an excitation energy of 7.1 MeV. As it emerges from the results displayed in Fig. 3 of Ref. [45], and at variance with the pairing rotational scheme, the two-particle transfer cross section associated with the excited pairing vibrational state is of the same order of magnitude as that connecting the ground states.

Within this context, it could be intriguing to check whether the reaction ${ }^{106} \mathrm{Sn}(p, t){ }^{104} \mathrm{Sn}$ populates a $0^{+}$state at an excitation energy of the order of $7 \mathrm{MeV}$. This state, can be written within the (pairing vibration) harmonic approximation, as $\left|{ }^{104} \operatorname{Sn}\left(0^{+} ; 7.1 \mathrm{MeV}\right)\right\rangle=|a\rangle \otimes|a\rangle \otimes|a\rangle \otimes|r\rangle$. Namely, a four-phonon pairing vibrational state, where

$$
|a\rangle=\left|\operatorname{gs}\left({ }^{102} \mathrm{Sn}\right)\right\rangle ; \quad|r\rangle=\left|\operatorname{gs}\left({ }^{98} \mathrm{Sn}\right)\right\rangle .
$$

In other words, the reaction ${ }^{106} \mathrm{Sn}(p, t){ }^{104} \mathrm{Sn}\left(0^{+}, 7.1 \mathrm{MeV}\right)$ is, within the harmonic picture of pairing vibrations, equivalent to the reaction ${ }^{100} \mathrm{Sn}(p, t){ }^{98} \mathrm{Sn}(\mathrm{gs})$. It is of notice that a three-phonon pairing vibrational states has been observed [52] in the reaction ${ }^{204} \mathrm{~Pb}(t, p){ }^{206} \mathrm{~Pb}$ at an excitation energy of about $6 \mathrm{MeV}$, with $Q$ values and absolute differential cross sections compatible with the excitation of the ${ }^{208} \mathrm{~Pb}$ pair addition mode, namely ${ }^{208} \mathrm{~Pb}(t, p){ }^{210} \mathrm{~Pb}(\mathrm{gs})$. While in this case deviations from the harmonic prediction are modest (essentially, most of them arising from the presence of the valence orbital $p_{1 / 2}$ lying just below $\epsilon_{F}\left({ }^{208} \mathrm{~Pb}\right)$ [53]), in the case of pairing vibrations based on ${ }^{100} \mathrm{Sn}$, anharmonicities are expected to be much stronger. This is in keeping with the fact that $N=Z$ nuclei display, as a rule, coexistence phenomena. That is, a strong competition between spherical and deformed $0^{+}$states (cf., e.g., Refs. [54-56] and references therein; see also Ref. [57]).

\section{CONCLUSIONS}

The microscopic nuclear structure (BCS) description of pairing rotational bands, together with the second-order DWBA description of two-nucleon transfer reactions which include successive, simultaneous, and non-orthogonal channels provide, arguably without free parameters, an overall account of Cooper pair transfer to superfluid nuclei. Inarguably, theory not only reproduces all reported ${ }^{A+2} \operatorname{Sn}(p, t)^{A} \operatorname{Sn}(\mathrm{gs})$ absolute cross-section data within experimental errors, but it does so with uncertainties below the $10 \%$ level.

The study of the pairing vibrational scheme around the starting and end points of the pairing rotational spectrum promises to provide new insight on pairing fluctuations and their anharmonicities, in situations of large neutron excess and of $N \sim Z$, i.e., around closed-shell system ${ }^{132} \mathrm{Sn}$ and of the likely deformation coexistent ${ }^{100} \mathrm{Sn}$, respectively.

\section{APPENDIX A: PAIR SPIN AND DOMAIN WALL}

In what follows we discuss the mean-field properties of the Hamiltonian

$$
H_{p}-\lambda N=H_{\mathrm{sp}}^{\prime}+V_{p},
$$

where

$$
H_{\mathrm{sp}}^{\prime}=\sum_{\nu>0} \epsilon_{\nu} N_{\nu}
$$

with

$$
\epsilon_{v} \equiv \varepsilon_{v}-\lambda
$$

and

$$
N_{v}=a_{\nu}^{\dagger} a_{v}+a_{\bar{v}}^{\dagger} a_{\bar{v}} .
$$

The pairing interaction is defined as

$$
V_{p}=-G P^{\dagger} P,
$$

where

$$
P^{\dagger}=\sum_{\nu>0} P_{v}^{\dagger}
$$

and

$$
P_{v}^{\dagger}=a_{v}^{\dagger} a_{\bar{\nu}}^{\dagger} .
$$

The single-particle Hamiltonian $H_{\mathrm{sp}}^{\prime}$ is invariant under time-reversal operations. As a consequence, orbitals are twofold degenerate, the corresponding states being denoted $|v\rangle$ and $|\bar{v}\rangle$.

Because the operators $N_{\nu}, P_{\nu}^{\dagger}$, and $P_{\nu}$ satisfy the commutation relations

$$
\begin{aligned}
{\left[P_{v}^{\dagger}, P_{v}\right] } & =N_{v}-1, \\
{\left[N_{v}-1, P_{v}^{\dagger}\right] } & =2 P_{v}^{\dagger},
\end{aligned}
$$

and

$$
\left[N_{v}-1, P_{v}\right]=-2 P_{v},
$$

one can define the $x, y$, and $z$ components of the pairspin operator $\vec{s}(v)$ according to the relations,

$$
\begin{aligned}
& s_{x}(v)=\frac{1}{2}\left(P_{v}^{\dagger}+P_{v}\right)=\frac{1}{2}\left(\begin{array}{ll}
0 & 1 \\
1 & 0
\end{array}\right), \\
& s_{y}(v)=\frac{1}{2 i}\left(P_{v}^{\dagger}-P_{v}\right)=\frac{1}{2}\left(\begin{array}{cc}
0 & -i \\
i & 0
\end{array}\right),
\end{aligned}
$$

and

$$
s_{z}(v)=\frac{1}{2}\left(N_{v}-1\right)=\frac{1}{2}\left(\begin{array}{cc}
1 & 0 \\
0 & -1
\end{array}\right) .
$$

In fact, using the commutation relations (A8)-(A10) stated above one obtains

$$
\begin{aligned}
& {\left[s_{x}(v), s_{y}(v)\right]=i s_{z}(v),} \\
& {\left[s_{y}(v), s_{z}(v)\right]=i s_{x}(v),} \\
& {\left[s_{z}(v), s_{x}(v)\right]=i s_{y}(v) .}
\end{aligned}
$$

Of notice is that these $\vec{s} \equiv\left(s_{x}, s_{y}, s_{z}\right)$ operators although acting in an abstract, gauge space, are as real as the standard spin of electrons and nucleons. The $z$ component of pairspin 
(a)

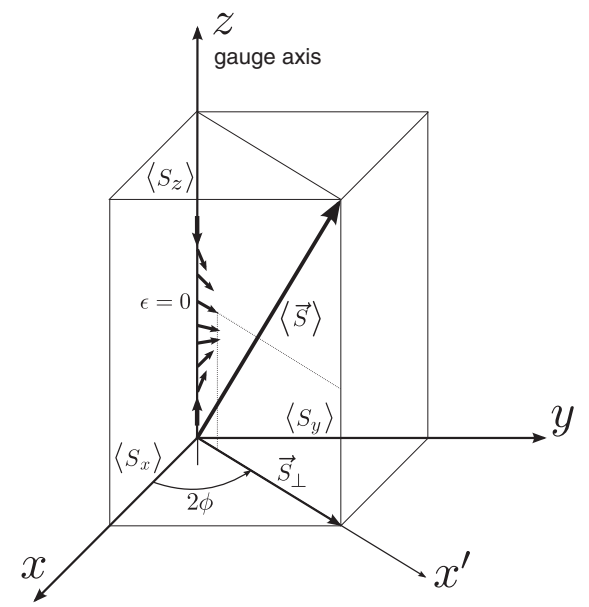

(b)

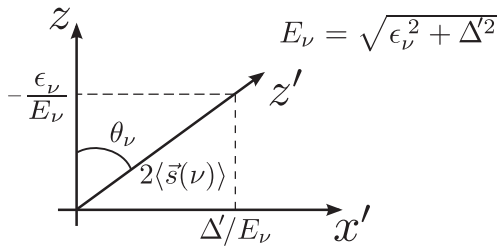

$\sin \theta_{\nu}=\frac{\Delta^{\prime}}{E_{\nu}}$

$\cos \theta_{\nu}=-\frac{\epsilon_{\nu}}{E_{\nu}}$

$|\langle\vec{s}(\nu)\rangle|=1 / 2$

(c)

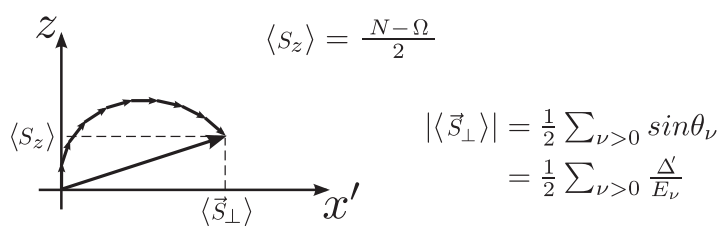

FIG. 11. (a) In the presence of the pairing interaction, the pairspin vector $\langle\vec{S}\rangle$ acquires a component in the $x, y$ plane. It is then possible to define an intrinsic system $\mathcal{K}^{\prime}$, which is obtained rotating the laboratory system $\mathcal{K}$ by the gauge angle $2 \phi$ around the $z$ (gauge) axis (positive angles correspond to counterclockwise rotations). The contributions from the individual pairspins [all lying in the $\left(z, x^{\prime}\right)$ plane] are schematically shown. The perpendicular component dominates for states close to the Fermi energy $(\epsilon \approx 0)$, while the pairspins associated with states far from $\varepsilon_{F}$ are aligned along the $z$ axis. (b) Contribution of a pairspin associated with single-particle states of energy $\epsilon_{v}$. The pairspin vector makes an angle $\theta_{v}$ with the gauge $z$ axis such that $\sin \theta_{v}=\Delta / E_{v}$ and $\cos \theta_{v}=-\epsilon_{v} / E_{v}$. (c) The total pairspin vector is the sum of many individual contributions. The value of its projection on the $z$ axis is equal to $(N-\Omega) / 2$, where $N$ is the number of particles and $\Omega$ is the total pair degeneracy of the single-particle subspace considered to describe the system [see Eq. (B18)].

pointing up means "occupied" twofold degenerate orbitals, pairspin pointing down means "empty," while a pairspin pointing sideways implies a certain phased linear combination of up and down (see Fig. 1 as well as Fig. 11). In keeping with this scenario, the BCS ground state displays a gradual rotation, such as a domain wall, of the pairspin vectors across the Fermi surface.

The eigenvectors of $s_{z}(v)$ in pairspin space are

$$
|1\rangle_{\nu}=\left(\begin{array}{l}
1 \\
0
\end{array}\right)_{v} \equiv a_{\nu}^{\dagger} a_{\bar{\nu}}^{\dagger}|0\rangle_{\nu}
$$

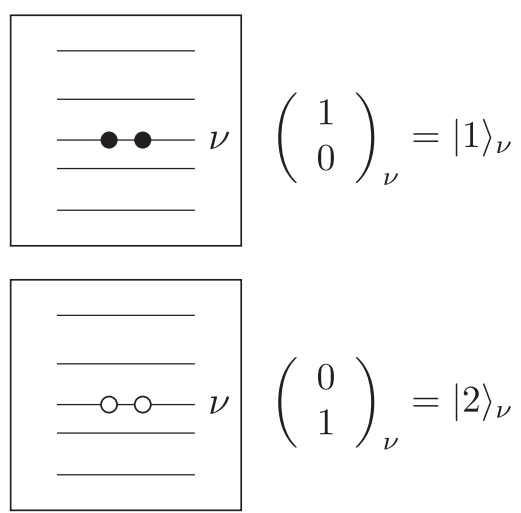

FIG. 12. Schematic representation of the eigenvectors of $s_{z}(v)$ in pairspin space: states $|1\rangle_{\nu}$ and $|2\rangle_{\nu}$ can be viewed as a pair addition and a pair removal mode, at the level of individual pairs of time-reversal states $(v, \bar{v})$.

and

$$
|2\rangle_{\nu}=\left(\begin{array}{l}
0 \\
1
\end{array}\right)_{\nu} \equiv|0\rangle_{\nu}
$$

To better clarify the meaning of state $|1\rangle_{v}$ and $|2\rangle_{v}$, let us assume to be working with a set of twofold degenerate states $v_{1}, v_{2}, \ldots$, each pair of levels connected by time reversal, e.g., $\left(v_{1}, \bar{v}_{1}\right)$. In the uncorrelated case $(G=0),|1\rangle$ and $|2\rangle$ can be viewed as fully occupied or fully empty states (see Fig. 12), that is, a two-particle (filled) and a two-hole state (empty), respectively [see Eqs. (A17) and (A18) below; see also Eqs. (A22) and (A23)]. The same argumentation can be applied to each pair of $(m,-m)$ states connected by time reversal, of a general set of $(2 j+1)$ degenerate single-particle states. In other words, the system under consideration displays its pair addition and pair removal modes, at the level of individual pairs of time-reversal states $(v, \bar{v})$, building blocks from which Cooper pairs are built. Within this context, it is of notice that Cooper's model works equally well if one thinks of it in terms of a correlated two-hole state in the Fermi sea, BCS being an extension and, in a way, a natural melting of the two views, as required by quantum mechanics [zero-point fluctuations (ZPFs) which, within the present context can be interpreted in terms of ground-state correlations]. The quantal nature of these correlations is further evidenced by the fact that the different components enter the correlated Cooper pair in terms of probability amplitudes [see, in particular, Eq. (B17)].

In the basis (A16), the operators $s_{x}, s_{y}$, and $s_{z}$ have the same matrix representation as the Pauli matrices except for a factor $1 / 2$, that is, $\vec{\sigma}=2 \vec{s}$. The action of $s_{z}$ and $N$ on the states (A16) is given by

$$
\begin{aligned}
& s_{z}(v)\left(\begin{array}{l}
1 \\
0
\end{array}\right)_{v}=\frac{1}{2}\left(\begin{array}{rr}
1 & 0 \\
0 & -1
\end{array}\right)\left(\begin{array}{l}
1 \\
0
\end{array}\right)_{v}=1 / 2\left(\begin{array}{l}
1 \\
0
\end{array}\right)_{v} ; \\
& s_{z}(v)\left(\begin{array}{l}
0 \\
1
\end{array}\right)_{v}=\frac{1}{2}\left(\begin{array}{rr}
1 & 0 \\
0 & -1
\end{array}\right)\left(\begin{array}{l}
0 \\
1
\end{array}\right)_{v}=-1 / 2\left(\begin{array}{l}
0 \\
1
\end{array}\right)_{v} ; \\
& N_{\nu}\left(\begin{array}{l}
1 \\
0
\end{array}\right)_{v}=\left(\begin{array}{ll}
2 & 0 \\
0 & 0
\end{array}\right)\left(\begin{array}{l}
1 \\
0
\end{array}\right)_{v}=2\left(\begin{array}{l}
1 \\
0
\end{array}\right)_{v} ; \\
& N_{v}\left(\begin{array}{l}
0 \\
1
\end{array}\right)_{v}=\left(\begin{array}{ll}
2 & 0 \\
0 & 0
\end{array}\right)\left(\begin{array}{l}
0 \\
1
\end{array}\right)_{v}=0 .
\end{aligned}
$$


Inverting the relations (A11), the pair operators $P^{\dagger}$ and $P$ can be identified with the raising and lowering operator in pairspace. In fact,

$$
P^{\dagger}=\sum_{\nu>0}\left[s_{x}(v)+i s_{y}(v)\right]=S_{x}+i S_{y} \equiv S_{+},
$$

and

$$
P=\sum_{\nu>0}\left[s_{x}(v)-i s_{y}(\nu)\right]=S_{x}-i S_{y} \equiv S_{-} .
$$

In this space, i.e., the space subtended by the states $|1\rangle_{v}$ and $|2\rangle_{\nu}$, the operators $S_{+}(v)$ and $S_{-}(v)$ are represented by the matrices

$$
\begin{aligned}
& \left(\left\langle i\left|S_{+}(v)\right| j\right\rangle\right)=\left(\begin{array}{ll}
\left\langle 1\left|P_{\nu}^{\dagger}\right| 1\right\rangle & \left\langle 1\left|P_{\nu}^{\dagger}\right| 2\right\rangle \\
\left\langle 2\left|P_{\nu}^{\dagger}\right| 1\right\rangle & \left\langle 2\left|P_{\nu}^{\dagger}\right| 2\right\rangle
\end{array}\right)=\left(\begin{array}{ll}
0 & 1 \\
0 & 0
\end{array}\right), \\
& \left(\left\langle i\left|S_{-}(\nu)\right| j\right\rangle\right)=\left(\begin{array}{ll}
\left\langle 1\left|P_{\nu}\right| 1\right\rangle & \left\langle 1\left|P_{\nu}\right| 2\right\rangle \\
\left\langle 2\left|P_{\nu}\right| 1\right\rangle & \left\langle 2\left|P_{\nu}\right| 2\right\rangle
\end{array}\right)=\left(\begin{array}{ll}
0 & 0 \\
1 & 0
\end{array}\right),
\end{aligned}
$$

while

$$
\begin{aligned}
(\langle i|N(\nu)-1| j\rangle) & =\left(\begin{array}{lr}
\left\langle\nu \bar{v}\left|\left(N_{v}-1\right)\right| v \bar{v}\right\rangle & \left\langle\nu \bar{v}\left|\left(N_{v}-1\right)\right| 0\right\rangle \\
\left\langle 0\left|\left(N_{\nu}-1\right)\right| \nu \bar{v}\right\rangle & \left\langle 0\left|\left(N_{v}-1\right)\right| 0\right\rangle
\end{array}\right) \\
& =\left(\begin{array}{rr}
1 & 0 \\
0 & -1
\end{array}\right) .
\end{aligned}
$$

Consequently,

$$
\begin{aligned}
& P_{v}^{\dagger}\left(\begin{array}{l}
0 \\
1
\end{array}\right)_{v}=\left(\begin{array}{ll}
0 & 1 \\
0 & 0
\end{array}\right)\left(\begin{array}{l}
0 \\
1
\end{array}\right)_{v}=\left(\begin{array}{l}
1 \\
0
\end{array}\right)_{v} ; \\
& P_{v}^{\dagger}\left(\begin{array}{l}
1 \\
0
\end{array}\right)_{v}=\left(\begin{array}{ll}
0 & 1 \\
0 & 0
\end{array}\right)\left(\begin{array}{l}
1 \\
0
\end{array}\right)_{v}=0,
\end{aligned}
$$

while

$$
\begin{aligned}
& P_{\nu}\left(\begin{array}{l}
0 \\
1
\end{array}\right)_{v}=\left(\begin{array}{ll}
0 & 0 \\
1 & 0
\end{array}\right)\left(\begin{array}{l}
0 \\
1
\end{array}\right)_{v}=0, \\
& P_{\nu}\left(\begin{array}{l}
1 \\
0
\end{array}\right)_{v}=\left(\begin{array}{ll}
0 & 0 \\
1 & 0
\end{array}\right)\left(\begin{array}{l}
1 \\
0
\end{array}\right)_{v}=\left(\begin{array}{l}
0 \\
1
\end{array}\right)_{v} .
\end{aligned}
$$

The total pairspin in the $z$ direction is closely related to the number operator

$$
S_{z}=\sum_{v>0} s_{z}(v)=\frac{1}{2}(N-\Omega),
$$

where $N=\sum_{v>0} N_{\nu}$, and $\Omega$ is the total number of twofold degenerated single-particle orbitals, associated with the singleparticle space considered. Within this context, see Eq. (B18).

The two terms of the pairing Hamiltonian can now be rewritten as

$$
H_{\mathrm{sp}}^{\prime}=\sum_{\nu>0} \epsilon_{\nu} N_{\nu}=\sum_{\nu>0} \epsilon_{\nu}\left[1+2 s_{z}(\nu)\right]
$$

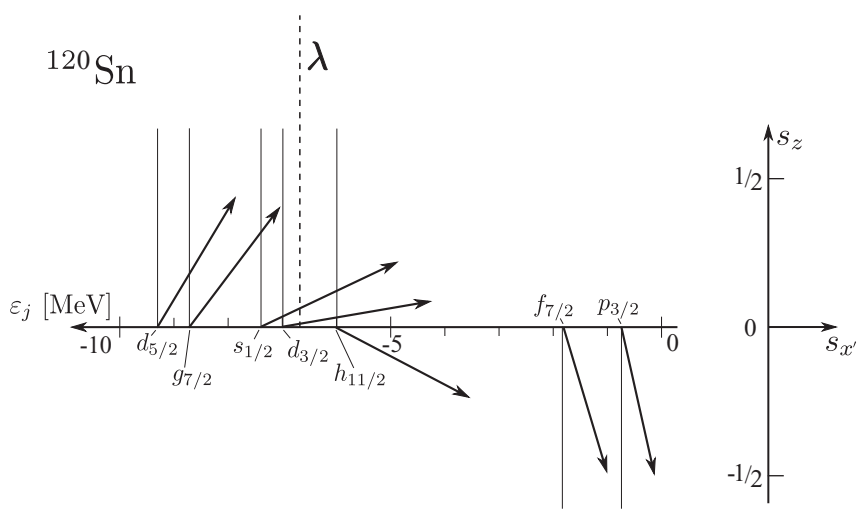

FIG. 13. Pairspin distribution associated with the seven valence single-particle orbitals lying around the Fermi energy of ${ }^{120} \mathrm{Sn}$. The calculations were carried out making use of the results displayed in Table II, in particular the values $U_{v}^{\prime} V_{v}^{\prime}$, following the prescription discussed in the caption to Fig. 5.

and

$$
\begin{aligned}
V_{p}= & -\sum_{\nu_{1}, v_{2}>0} G P_{\nu_{1}}^{\dagger} P_{\nu_{2}} \\
= & -G\left[\sum_{\nu_{1}>0} s_{x}\left(v_{1}\right) \sum_{\nu_{2}>0} s_{x}\left(\nu_{2}\right)+\sum_{\nu_{1}>0} s_{y}\left(v_{1}\right) \sum_{\nu_{2}>0} s_{y}\left(\nu_{2}\right)\right] \\
& -G \sum_{\nu_{1}>0} s_{z}\left(v_{1}\right) \\
= & -G \sum_{\nu_{1}, \nu_{2}>0}\left(\vec{s}_{\perp}\left(v_{1}\right) \cdot \vec{s}_{\perp}\left(v_{2}\right)\right)-G \sum_{\nu_{1}>0} s_{z}\left(v_{1}\right)
\end{aligned}
$$

where $s_{\perp}(v)=s_{x}(v) \hat{i}+s_{y}(v) \hat{j}, \hat{i}$ and $\hat{j}$ being unit vectors along the $x$ and $y$ directions, respectively. The last term is the contribution of the pairing interaction to the single-particle mean field $H_{\mathrm{sp}}$. Although it can easily be incorporated in $H_{s p}$, it is customary to neglect it, in keeping with the schematic nature of $V_{p}$, tailored to act on the pair space. However, this contribution is to be considered when comparing the solution of schematic models with exact solutions (see, e.g., Refs. [58,59]).

The interaction $V_{p}$ is a spin-spin coupling, which seeks to align the transverse components of the pairspins. Was it not for $H_{\mathrm{sp}}$, which depends on $S_{z}$, all pairspins would line up in the same direction (strong coupling limit), perpendicular to the $z$ axis, in keeping with the fact that $V_{p}$ is a function of only the $S_{x}$ and $S_{y}$ pairspin operator. In the opposite limit, that is, for $V_{p}=$ 0 , pairspin alignment is zero, that is, there is no component of the pairspin in the $(x, y)$ plane perpendicular to the $z$ axis [see Fig. 1(a)]. From this figure it is clear that $H_{\text {sp }}$ counteracts pair spin alignment. Indeed $H_{\text {sp }}$ plays, in the nucleus, the role of a magnetic field in a solid, which tends to align the spins in the $z$ direction, or opposite to it, with a strength (as measured by $\epsilon_{\nu}$ ) that increases in absolute value as a function of the energy of the twofold degenerate levels $(v, \bar{v})$ away from the Fermi energy $\varepsilon_{F}=\lambda$. Thus, for sufficiently large values of $\left|\varepsilon_{v}-\epsilon_{F}\right|$, of the order of $2 \Delta(2-3 \mathrm{MeV})$, the single-particle energy as measured by $H_{\mathrm{sp}}$ dominates [see Fig. 1 as well as Fig. 11(a) and 13]. This is also the reason why calculations of pairing 
correlations in nuclei, which depend on small contributions arising from orbitals distant from the Fermi energy are to be handled with care, in particular when comparing the calculated results with the experimental findings. Within this context, see Sec. IV A, discussion connected with $\alpha_{0}^{\prime}\left(\vec{r}_{1}, \vec{r}_{2}\right)$. Close to the Fermi energy, $V_{p}$ is the overriding effect, and a large transverse pairspin is expected, as testified by the coherent sums in Eq. (A27). While any single pairspin in these sums displays large quantal fluctuations, the total pairspin has a well defined magnitude and orientation, because the fluctuations of the constituent pairspins add quadratically.

The pairing Hamiltonian can be diagonalized in the meanfield approximation, substituting one of the sums in Eq. (A27) with its average value in the mean-field ground state. Let us assume that the average value of the pairspin polarization vector $\left|\left\langle\vec{S}_{\perp}\right\rangle\right|={ }_{\mathcal{K}^{\prime}}\left\langle\mathrm{BCS}\left|S_{\perp}\right| \mathrm{BCS}\right\rangle_{\mathcal{K}^{\prime}}$ [see. Eq. (A51) below] is nonzero, and choose a definite orientation for it in the $x-y$ plane (denoted $x^{\prime}$ ), subtending an angle $2 \phi$ with the $x$ axis (definition of the body-fixed, intrinsic frame; see Fig. 11). The pair interaction is then replaced by a mean field, namely the pair field of strength $\Delta^{\prime} \equiv G\left|\left\langle\vec{S}_{\perp}\right\rangle\right|$. One can then write

$$
\begin{aligned}
U_{p} & =-2 G\left|\left\langle\vec{S}_{\perp}\right\rangle\right| \sum_{\nu>0}\left[s_{x}(v) \cos 2 \phi+s_{y}(v) \sin 2 \phi\right] \\
& =-2 \Delta^{\prime} \sum_{\nu>0}\left[s_{x}(\nu) \cos 2 \phi+s_{y}(v) \sin 2 \phi\right] .
\end{aligned}
$$

Making use of the relation given in Eq. (A11) one finds

$$
\begin{aligned}
\Delta^{\prime}\left[s_{x}(v) \cos 2 \phi+s_{y}(v) \sin 2 \phi\right] & =\frac{1}{2}\left(P_{\nu}^{\dagger} \Delta^{\prime} e^{-2 i \phi}+P_{v} \Delta^{\prime} e^{2 i \phi}\right) \\
& =\frac{1}{2}\left(P_{v}^{\dagger} \Delta+P_{\nu} \Delta^{*}\right), \quad \text { (A29) }
\end{aligned}
$$

where we have introduced $\Delta=e^{-2 i \phi} \Delta^{\prime}$. Consequently,

$$
U_{p}=-\Delta^{\prime} \sum_{\nu>0}\left(P_{\nu}^{\dagger^{\prime}}+P_{v}^{\prime}\right)=-\sum_{\nu>0}\left(P_{v}^{\dagger} \Delta+P_{\nu} \Delta^{*}\right),
$$

$P^{\prime \dagger}, P^{\prime}$ denoting the operators in the body-fixed frame of axis $\left(x^{\prime}, y^{\prime}\right)$ [cf. Fig. 11(a) and Appendix B] in which, by definition, $\phi=0$; that is $\left\langle S_{y}^{\prime}\right\rangle=0$ and $\left\langle S_{x}^{\prime}\right\rangle=\left\langle S_{\perp}^{\prime}\right\rangle=\alpha_{0}^{\prime}$.

The total Hamiltonian then becomes a sum over individual pairspins,

$$
\left(H_{p}\right)_{\mathrm{MF}}=\sum_{\nu>0} h_{\nu}
$$

where

$$
\begin{aligned}
h_{v}= & \epsilon_{v}+\epsilon_{v}\left(\begin{array}{rr}
1 & 0 \\
0 & -1
\end{array}\right) \\
& -\Delta^{\prime}\left(\begin{array}{cc}
0 & \cos 2 \phi-i \sin 2 \phi \\
\cos 2 \phi+i \sin 2 \phi & 0
\end{array}\right) .
\end{aligned}
$$

It is of notice that thinking in terms of the independent (quasiparticle) densities the first and second term are connected with the normal and the third with the so-called abnormal density, leading to ODLRO.

Within this context, in the mean field associated with the (diagonal) first term of the above equation [see also Eq. (A2)], the particles that move independently of each other are nucleons. For this to happen, all the nucleons must participate in a highly coherent ballet following a refined choreography, in such a way that each dancer moves as if he was alone in the scene, being fenced in through a dancers-like wall only when approaching the edges of the scene. As has been stated in the literature [60], it is a "rather unfortunate perversity" that views independent particle motion as antithetic to nuclear collective motion.

In the case of the mean field described by the second term of Eq. (A32), the entities that play the role of nucleons in the case above are now pairspins [pair addition and pair removal $(v, \bar{v}$ modes)]. The only circumstance in which pairspins feel the pushings and pullings of the other pairspins is when they try to adopt a different orientation but that defined by $S_{\perp}$ (i.e., $x^{\prime}$ direction, see Fig. 11), being forced to align back by a domain wall. In the present case, the ballet is not performed by single dancers in a scene, but by couples in a crowded dancing hall. In spite of such a less cultured setup, the choreography is even more refined than previously described. This is because the partners of each dancing couple can, not only when close to each other, but also when finding themselves at opposite extremes of the dancing hall (coherence length), follow the other partners' moves without missing a single step. Such a choreography of strongly overlapping pairs translates, in the gauge (pairspin) space, into the definition of a privileged orientation.

This can be better seen by expressing the diagonalization condition (4), namely

$$
\sum_{\nu>0} \epsilon_{v} N_{v}-\Delta^{\prime}\left(P^{\prime \dagger}+P^{\prime}\right)+\frac{\Delta^{\prime 2}}{G}=\sum_{\nu>0} E_{\nu} \tilde{N}_{\nu}+\text { const }
$$

in terms of the quasispin operators (A11) and (A12) and

$$
s_{z^{\prime}}(v)=-\frac{1}{2}\left(\tilde{N}_{\nu}-1\right) \text {. }
$$

That is,

$$
\begin{aligned}
\sum_{\nu>0} & {\left[\epsilon_{\nu} 2 s_{z}(\nu)-\Delta^{\prime} 2 s_{x}(\nu)\right]+\sum_{\nu>0}\left(\epsilon_{\nu}-E_{\nu}\right)+\frac{\Delta^{\prime 2}}{G} } \\
= & -\sum_{\nu>0} E_{\nu} 2 s_{z^{\prime}}(\nu)+\text { const. }
\end{aligned}
$$

In a similar way in which $s_{z}(v)$ is diagonal in the independent (pair) particle situation, $s_{z^{\prime}}(v)$ is diagonal in the quasiparticle representation, with eigenvalues $1 / 2$ when acting on the corresponding occupied states $(|\mathrm{HF}\rangle$ and $|\mathrm{BCS}\rangle$ states, respectively) and $-1 / 2$ when acting on the corresponding unoccupied states (levels above $\varepsilon_{F}$ and two quasiparticle states, respectively). Equating the (quasispin) operator terms and the $c$-number terms one obtains

$$
\text { const }=\sum_{\nu>0}\left(\epsilon_{\nu}-E_{v}\right)+\frac{\Delta^{\prime 2}}{G},
$$

and

$$
-\frac{\epsilon_{v}}{E_{v}} s_{z}(v)+\frac{\Delta^{\prime}}{E_{v}} s_{x}(v)=s_{z^{\prime}}(v)
$$

The above equation determines the angle $\theta_{v}$ in the $z-x$ plane, which leads to independent quasispin motion. In the 
strong coupling limit $\left(\left|\Delta^{\prime}\right| \gg\left|\epsilon_{\nu}\right|\right)$, "magnetization" is total, all pairspins [pair addition and removal $(v, \bar{v})$ pairs] pointing along the $x$ direction.

As already stated in connection with Eq. (4) of the text, the $c$-number const is equal to the ground-state energy, also known as the $U$ term of $\left(H_{p}\right)_{\mathrm{MF}}$ (see, e.g., Ref. [10], Eq. (G11) of Appendix G; see also below, section on ground-state energy and pairing correlation energy).

In keeping with the fact that the quasispin states are normalized, and that one has chosen a representation in which $s_{y}(v)=0$, the quasispin prefactors of Eq. (A34b) must fulfill the relation

$$
\left(-\frac{\epsilon_{v}}{E_{v}}\right)^{2}+\frac{\Delta^{\prime 2}}{E_{v}^{2}}=1
$$

It is of notice that the eigenvalue equation (A32),

$$
\epsilon_{v}-\left(\begin{array}{cc}
-\epsilon_{\nu} & \Delta^{\prime} e^{-2 i \phi} \\
\Delta^{\prime} e^{2 i \phi} & \epsilon_{v}
\end{array}\right)\left(\begin{array}{c}
V_{v} \\
U_{v}
\end{array}\right)=\left(\epsilon_{v}-E\right)\left(\begin{array}{c}
V_{v} \\
U_{v}
\end{array}\right),
$$

has the solutions $\pm E_{v}$, where [see also Eq. (A35)]

$$
E_{v}=\sqrt{\epsilon_{v}^{2}+\Delta^{\prime 2}}
$$

The positive sign corresponds to the lowest total pairspin energy $\left(\epsilon_{v}-E_{v}\right)$, while the negative sign corresponds to the excited states (two-quasiparticle states; cf. section below on excited states). The relation between the $U_{v}$ and the $V_{v}$ components of the eigenvector can be deduced from Eq. (A36),

$$
\begin{aligned}
-\epsilon_{v} V_{v}+\Delta^{\prime} e^{-2 i \phi} U_{v} & =E_{v} V_{v}, \\
\Delta^{\prime} e^{-2 i \phi} V_{v}+\epsilon_{v} U_{v} & =E_{v} U_{v} .
\end{aligned}
$$

Equation (A38) leads to $\left(E_{v}+\epsilon_{v}\right) V_{v}=\Delta^{\prime} e^{-2 i \phi} U_{v}$, implying a phase difference $-2 \phi$ between the $V_{v}$ and the $U_{v}$ occupation amplitudes. This allows one to write the following relations:

$$
\begin{aligned}
& U_{v}=U_{\nu}^{\prime} e^{i \phi}, \\
& V_{v}=V_{\nu}^{\prime} e^{-i \phi},
\end{aligned}
$$

with $U_{v}^{\prime}$ and $V_{v}^{\prime}$ being the moduli of $U_{v}$ and $V_{v}$, respectively. These quantities can be calculated from the square modulus of Eq. (A38)

$$
\left(\epsilon_{v}+E_{v}\right)^{2}\left|V_{v}\right|^{2}=\Delta^{\prime 2}\left|U_{v}\right|^{2} .
$$

Making use of the normalization relation $\left|V_{v}\right|^{2}=1-\left|U_{\nu}\right|^{2}$, one obtains

$$
U_{v}^{\prime 2}=\frac{1}{2}\left(1+\frac{\epsilon_{v}}{E_{v}}\right), \quad V_{v}^{\prime 2}=\frac{1}{2}\left(1-\frac{\epsilon_{v}}{E_{v}}\right) .
$$

Defining the angle $\theta_{v}$ according to

$$
\cos \theta_{v}=-\frac{\epsilon_{v}}{E_{v}}, \quad \sin \theta_{v}=\frac{\Delta^{\prime}}{E_{v}},
$$

one can rewrite the quasiparticle amplitudes as

$$
U_{v}^{\prime}=\sin \left(\theta_{v} / 2\right), \quad V_{v}^{\prime}=\cos \left(\theta_{v} / 2\right),
$$

where the angle $\theta_{v}$ represents the angle between the direction of $\vec{s}(v)\left[z^{\prime}\right.$ axis in Fig. 11(b)] and the $z$ axis. The average values of $s_{x}(v), s_{y}(v)$ calculated with these eigenfunctions, generalizations of the eigenvectors introduced before [see Eqs. (A16a) and (A16b)], are given by

$$
\begin{aligned}
\left\langle s_{x}(v)\right\rangle & =\left(V_{v}^{*}, U_{v}^{*}\right) s_{x}(v)\left(\begin{array}{c}
V_{v} \\
U_{v}
\end{array}\right)=\frac{1}{2}\left(U_{v} V_{v}^{*}+V_{v} U_{v}^{*}\right) \\
& =\sin \frac{\theta_{v}}{2} \cos \frac{\theta_{v}}{2} \cos 2 \phi=U_{v}^{\prime} V_{v}^{\prime} \cos 2 \phi=\frac{\Delta^{\prime}}{2 E_{v}} \cos 2 \phi, \\
\left\langle s_{y}(v)\right\rangle & =\left(V_{v}^{*}, U_{v}^{*}\right) s_{y}(v)\left(\begin{array}{c}
V_{v} \\
U_{v}
\end{array}\right)=\frac{i}{2}\left(U_{v} V_{v}^{*}-V_{v}^{*} U_{v}\right) \\
& =\sin \frac{\theta_{v}}{2} \cos \frac{\theta_{v}}{2} \sin 2 \phi=U_{v}^{\prime} V_{v}^{\prime} \sin 2 \phi=\frac{\Delta^{\prime}}{2 E_{v}} \sin 2 \phi,
\end{aligned}
$$

and

$$
\begin{aligned}
\left\langle s_{z}(v)\right\rangle & =\left(V_{v}^{*}, U_{v}^{*}\right) s_{z}(v)\left(\begin{array}{c}
V_{v} \\
U_{v}
\end{array}\right)=\frac{1}{2}\left(V_{v} V_{v}^{*}-U_{v}^{*} U_{v}\right) \\
& =\frac{1}{2}\left(\cos ^{2} \frac{\theta_{v}}{2}-\sin ^{2} \frac{\theta_{v}}{2}\right)=-\frac{1}{2}\left(U_{v}^{\prime 2}-V_{v}^{\prime 2}\right)=-\frac{\varepsilon_{v}}{2 E_{v}} .
\end{aligned}
$$

The dependence of $\left\langle s_{x}(v)\right\rangle$ and $\left\langle s_{y}(v)\right\rangle$ on the the angle $\phi$ is consistent with the ansatz (A28). Furthermore, making use of Eq. (A47), one can determine the modulus of $S_{\perp}$ self-consistently,

$$
\begin{aligned}
\left|\sum_{v>0}\left\langle\vec{s}_{\perp}(v)\right\rangle\right| & =\left|\left\langle\vec{S}_{\perp}\right\rangle\right|=\sum_{\nu>0}\left[\cos \frac{\theta_{v}}{2} \sin \frac{\theta_{v}}{2}\right] \\
& =\sum_{v>0} U_{v}^{\prime} V_{v}^{\prime}=\alpha_{0}^{\prime},
\end{aligned}
$$

a relation that is closely connected with the BCS gap equation. In Fig. 13 the pairspin distribution associated with the valence orbitals of ${ }^{120} \mathrm{Sn}$, calculated making use of the results collected in Table II and the scheme discussed in the caption to Fig. 5, is displayed.

The wave function describing the ground state of the system is the product of all pairspins, each of which is a linear combination of the pair addition and removal $(v, \bar{v})$ modes with the corresponding weights $V_{\nu}$ and $U_{\nu}$, respectively, amplitudes that define their alignment in gauge space. That is,

$$
\begin{aligned}
& \prod_{\nu>0}\left(U_{v}|2\rangle_{\nu}+V_{v}|1\rangle_{v}\right) \\
& =\prod_{\nu>0}\left[U_{v}\left(\begin{array}{l}
0 \\
1
\end{array}\right)+V_{\nu}\left(\begin{array}{c}
1 \\
0
\end{array}\right)\right] \\
& =\prod_{\nu>0}\left(U_{v}+V_{\nu} a_{\nu}^{\dagger} a_{\bar{v}}^{\dagger}\right)|0\rangle \\
& =\prod_{\nu>0}\left(e^{i \phi} U_{\nu}^{\prime}+e^{-i \phi} V_{\nu}^{\prime} a_{\nu}^{\dagger} a_{\bar{\nu}}^{\dagger}\right)|0\rangle \\
& =e^{i \Omega \phi} \prod_{\nu>0}\left(U_{v}^{\prime}+V_{\nu}^{\prime} e^{-2 i \phi} a_{\nu}^{\dagger} a_{\bar{\nu}}^{\dagger}\right)|0\rangle .
\end{aligned}
$$




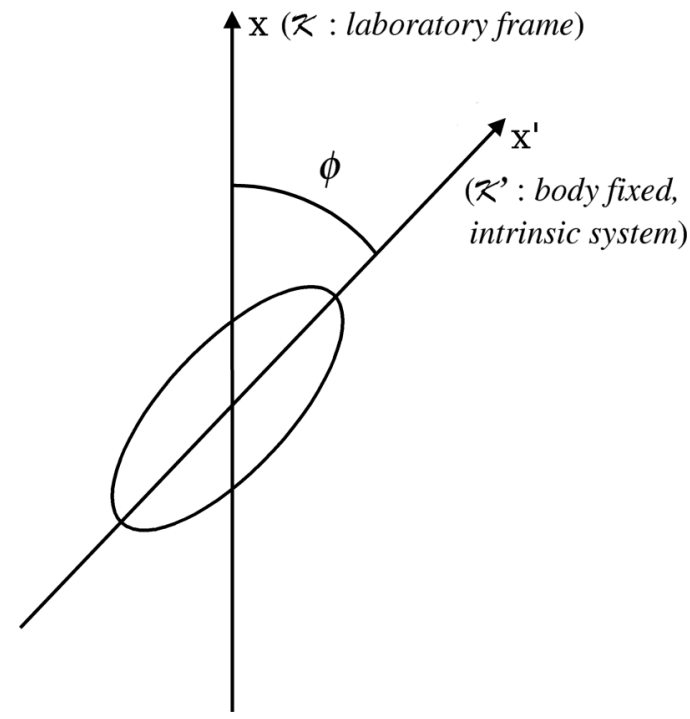

FIG. 14. Schematic representation of the deformation in gauge space associated with a superfluid nucleus leading to pairspin alignment (see also Fig. 11).

Leaving out the overall phase one can write

$$
\begin{aligned}
|\mathrm{BCS}(\phi)\rangle_{\mathcal{K}} & =\prod_{\nu>0}\left(U_{v}^{\prime}+V_{v}^{\prime} e^{-2 i \phi} a_{\nu}^{\dagger} a_{\bar{\nu}}^{\dagger}\right)|0\rangle \\
& =\prod_{\nu>0}\left(U_{v}^{\prime}+V_{\nu}^{\prime} a_{\nu}^{\prime \dagger} a_{\bar{\nu}}^{\prime \dagger}\right)|0\rangle \\
& =|\mathrm{BCS}(\phi=0)\rangle_{\mathcal{K}^{\prime}},
\end{aligned}
$$

where $\mathcal{K}$ and $\mathcal{K}^{\prime}$ denote the laboratory and the body-fixed, intrinsic frame which, by definition, has $\phi=0$ (see Fig. 14).

The creation operator in the intrinsic, body-fixed frame of reference is

$$
a_{v}^{\prime \dagger}=\mathcal{G} a_{v}^{\dagger} \mathcal{G}^{-1}=e^{-i \phi} a_{v}^{\dagger},
$$

where $\mathcal{G}=e^{-i N \phi}$ is the gauge operator inducing rotations in the two-dimensional gauge space. The quantities $U_{v}^{\prime}$ and $V_{v}^{\prime}$ are real.

In the independent particle limit, that is,

$$
\begin{aligned}
\lim _{\Delta \rightarrow 0} & \prod_{\nu>0}\left(U_{\nu}+V_{\nu} a_{\nu}^{\dagger} a_{\bar{\nu}}^{\dagger}|0\rangle\right) \\
\quad & =\prod_{\nu>0, \nu<\nu_{F}} a_{\nu}^{\dagger} a_{\bar{\nu}}^{\dagger}|0\rangle=a_{\nu_{1}}^{\dagger} a_{\bar{\nu}_{1}}^{\dagger} a_{\nu_{2}}^{\dagger} a_{\bar{\nu}_{2}}^{\dagger} \cdots a_{\nu_{N}}^{\dagger} a_{\bar{\nu}_{N}}^{\dagger}|0\rangle \\
& =\frac{1}{\sqrt{2 N !}} \operatorname{det}(1 \overline{1}, 2 \overline{2}, \ldots, N \bar{N})|0\rangle,
\end{aligned}
$$

as expected.

Summing up, the instability of the Fermi surface associated with transverse pairspin polarization is associated with a manybody wave function, product of the individual pairspin states,

$$
\begin{aligned}
|0\rangle_{\nu} & =U_{v}\left|s_{z}(v)=-1 / 2\right\rangle+V_{v}\left|s_{z}(v)=+1 / 2\right\rangle \\
& =U_{\nu}|2\rangle_{\nu}+V_{v}|1\rangle_{\nu},
\end{aligned}
$$

superposition of pairspin up and down and, therefore, is nonaxially symmetric with respect to the gauge axis ( $z$ axis). In other words, a linear combination of pair addition and substraction modes mixed at the level of individual pairs of time-reversal states $(v, \bar{v})$ (see Fig. 12). In the same way as $\left|{ }^{208} \mathrm{~Pb}(\mathrm{gs})\right\rangle$ spends part of the time in the state $\left|{ }^{210} \mathrm{~Pb}(\mathrm{gs})\right\rangle$ and part in $\left|{ }^{206} \mathrm{~Pb}(\mathrm{gs})\right\rangle,\left|{ }^{120} \mathrm{Sn}(\mathrm{gs})\right\rangle$ is a mixture of pair addition and removal states $|1\rangle$ and $|2\rangle$, respectively. The transverse polarization-which inherently breaks gauge symmetryarises from such a superposition, a phenomenon that is produced by the action of the "external" mean pair field $U_{p}$.

The pairspin polarization may rotate collectively about the $z$-gauge axis. The azimuthal angle is therefore a dynamical variable (pairing rotations). The static pair field constitutes a deformation that defines an orientation. Through this deformation, the system spontaneously breaks away from axial symmetry, and the indeterminacy in the number of particles in a pair correlated state is an inherent feature of this symmetry breaking. The static deformation introduces a collective degree of freedom $\phi$ and gives the system the ability to rotate as a whole around the gauge axis.

When the mean field solution leads to $\left\langle\vec{S}_{\perp}\right\rangle=0$, the intrinsic motion has axial symmetry and hence conserves particle number. In these systems, gauge invariance can also be broken dynamically, a phenomenon that gives rise to the pairing vibrational spectrum observed around closed-shell nuclei, in terms of highly enhanced, single Cooper pair tunneling processes.

\section{Excited states}

The BCS equation in the body-fixed frame,

$$
\left(\begin{array}{cc}
\epsilon_{v} & -\Delta^{\prime} \\
-\Delta^{\prime} & -\epsilon_{v}
\end{array}\right)\left(\begin{array}{c}
V_{v}^{\prime} \\
U_{v}^{\prime}
\end{array}\right)=-E\left(\begin{array}{c}
V_{v}^{\prime} \\
U_{v}^{\prime}
\end{array}\right)
$$

leads to the eigenvalue equation

$$
\left(\epsilon_{v}+E\right)\left(\epsilon_{v}-E\right)-\Delta^{\prime 2}=0,
$$

with eigenvalues $E_{v}=\sqrt{\epsilon_{v}^{2}+\Delta^{\prime 2}}$, corresponding to the ground state previously considered [cf. Eq. (A37)] and $-E_{v}$, corresponding to excited states. The associated eigenvectors are

$$
|\mathrm{gs}\rangle=\left(\begin{array}{c}
V_{v}^{\prime} \\
U_{v}^{\prime}
\end{array}\right), \quad|\mathrm{exc}\rangle=\left(\begin{array}{c}
U_{v}^{\prime} \\
-V_{v}^{\prime}
\end{array}\right)
$$

The state $\mid$ exc $\rangle$ is a two-quasiparticle state. It can be excited acting with $\alpha_{\nu}^{\dagger} \alpha_{\bar{\nu}}^{\dagger}$ on $|\mathrm{BCS}\rangle_{\mathcal{K}^{\prime}}$. In fact, using $\alpha_{\nu}^{\dagger}=U_{v}^{\prime} a_{v}^{\prime \dagger}-V_{\nu}^{\prime} a_{\bar{\nu}}^{\prime}$ one finds [it is of notice that $s_{z}^{\prime}(v)=s_{z}(v)$ ]

$$
\begin{aligned}
\alpha_{v}^{\dagger} \alpha_{\bar{v}}^{\dagger} & =U_{v}^{\prime 2} P_{v}^{\prime \dagger}-V_{v}^{\prime 2} P_{v}^{\prime \dagger}+2 U_{v}^{\prime} V_{v}^{\prime} s_{z}(v) \\
& =\left(\begin{array}{cc}
U_{v}^{\prime} V_{v}^{\prime} & U_{v}^{\prime 2} \\
-V_{v}^{\prime 2} & -U_{v}^{\prime} V_{v}^{\prime}
\end{array}\right),
\end{aligned}
$$

and

$$
\begin{aligned}
\alpha_{\bar{v}} \alpha_{v} & =U_{v}^{\prime 2} P_{v}^{\prime}-V_{v}^{\prime 2} P_{v}^{\prime \dagger}+2 U_{v}^{\prime} V_{v}^{\prime} s_{z}^{\prime}(v) \\
& =\left(\begin{array}{cc}
U_{v}^{\prime} V_{v}^{\prime} & -V_{v}^{\prime 2} \\
U_{v}^{\prime 2} & -U_{v}^{\prime} V_{v}
\end{array}\right) .
\end{aligned}
$$


Making use of Eqs. (A23) and (A24), one finds that

$$
\begin{aligned}
\alpha_{\nu}^{\dagger} \alpha_{\bar{\nu}}^{\dagger}|\mathrm{gs}\rangle & =\alpha_{\nu}^{\dagger} \alpha_{\bar{\nu}}^{\dagger}\left(\begin{array}{c}
V_{v}^{\prime} \\
U_{v}^{\prime}
\end{array}\right)=\left(\begin{array}{cc}
U_{v}^{\prime} V_{v}^{\prime} & U_{v}^{\prime 2} \\
-V_{v}^{\prime 2} & -U_{\nu}^{\prime} V_{v}^{\prime}
\end{array}\right)\left(\begin{array}{c}
V_{v}^{\prime} \\
U_{v}^{\prime}
\end{array}\right) \\
& =\left(\begin{array}{c}
U_{v}^{\prime} \\
-V_{v}^{\prime}
\end{array}\right)=|\mathrm{exc}\rangle,
\end{aligned}
$$

and

$$
\begin{aligned}
\alpha_{v}^{\dagger} \alpha_{\bar{\nu}}^{\dagger}|\mathrm{exc}\rangle & =\alpha_{v}^{\dagger} \alpha_{\bar{\nu}}^{\dagger}\left(\begin{array}{c}
U_{v}^{\prime} \\
-V_{v}^{\prime}
\end{array}\right) \\
& =\left(\begin{array}{cc}
U_{v}^{\prime} V_{v}^{\prime} & U_{v}^{\prime 2} \\
-V_{\nu}^{\prime \prime 2} & -U_{v}^{\prime} V_{v}^{\prime}
\end{array}\right)\left(\begin{array}{c}
U_{v}^{\prime} \\
-V_{v}^{\prime}
\end{array}\right)=0 .
\end{aligned}
$$

Analogously, one finds

$$
\alpha_{\bar{\nu}} \alpha_{v}|\mathrm{gs}\rangle=0,
$$

and

$$
\alpha_{\bar{\nu}} \alpha_{v}|\mathrm{exc}\rangle=|\mathrm{gs}\rangle .
$$

We also remark that

$$
2 i s_{y}^{\prime}(v)=P_{\nu}^{\prime \dagger}-P_{\nu}^{\prime}=\alpha_{\nu}^{\dagger} \alpha_{\bar{\nu}}^{\dagger}-\alpha_{\bar{\nu}} \alpha_{\nu} .
$$

One can then show that the operator $2 i s_{y}^{\prime}(v)$ acting once on the ground state produces the excited state and, acting twice gives back the ground state, but for a sign change, that is,

$$
2 i s_{y}^{\prime}(v)|\mathrm{gs}\rangle=|\mathrm{exc}\rangle, \quad 2 i s_{y}^{\prime}(v)|\mathrm{exc}\rangle=-|\mathrm{gs}\rangle .
$$

Thus, two-quasiparticle excitation is equivalent to a rotation of the intrinsic system by an angle $\pi$ around the $y$ axis. This is in keeping with the fact that $e^{\pi i S_{y}}=2 i S_{y}$.

\section{Ground-state energy and correlations}

The energy of the ground state of the total system at the mean field level is obtained as the sum of the energy of each pair spin, $\epsilon_{v}-E_{v}$ [cf. Eq. (A36)], which is the eigenvalue diagonalizing the terms $\epsilon_{v} N_{v}-\left(P^{\dagger} \Delta+P \Delta^{*}\right)$ of the meanfield Hamiltonian, plus the constant term $\frac{\Delta^{\prime 2}}{G}$ [cf. Eq. (4)],

$$
E_{\mathrm{gs}}=\sum_{\nu>0}\left(\epsilon_{v}-E_{v}\right)+\frac{\Delta^{\prime 2}}{G} .
$$

In the case of the noninteracting system $(\Delta \rightarrow 0) E_{v} \rightarrow\left|\epsilon_{v}\right|$, so that the system's ground-state energy reads

$$
E_{\mathrm{gs}}^{0}=\sum_{\nu>0}\left(\epsilon_{\nu}-\left|\epsilon_{\nu}\right|\right)
$$

Consequently, the contributions corresponding to $\epsilon_{v}>0$ (i.e., $\left.\varepsilon_{v}>\lambda\right)$, cancel out, remaining only those of the states below the Fermi energy $\left(\epsilon_{v}<0\right)$. Thus,

$$
E_{\mathrm{gs}}^{0}=2 \sum_{\nu>0 ; \epsilon_{v}<0} \epsilon_{\nu},
$$

in keeping with the fact that in the present case all the pairs below the Fermi energy fully occupied. An important quantity characterizing superfluid systems is the so-called correlation energy, $E_{\text {corr }}$. It is defined as the difference between the energy of the interacting system and that of the noninteracting one,

$$
\begin{aligned}
E_{\text {corr }} & =E_{\mathrm{gs}}-E_{\mathrm{gs}}^{0} \\
& =\sum_{\nu>0}\left(\epsilon_{\nu}-E_{\nu}\right)+\frac{\Delta^{\prime 2}}{G}-2 \sum_{\nu>0 ; \epsilon_{\nu}<0} \epsilon_{\nu} .
\end{aligned}
$$

Collecting the contribution arising from levels displaying $\epsilon_{v}>$ 0 and $\epsilon_{v}<0$, the above equation can be written as

$$
\begin{aligned}
E_{\text {corr }} & =\sum_{\nu>0 ; \epsilon_{v}>0}\left(\epsilon_{\nu}-E_{\nu}\right)+\sum_{\nu>0 ; \epsilon_{v}<0}\left(-\epsilon_{\nu}-E_{\nu}\right)+\frac{\Delta^{\prime 2}}{G} \\
& =\sum_{\nu>0 ; \epsilon_{v}>0}\left(\epsilon_{\nu}-E_{\nu}\right)+\sum_{\nu>0 ; \epsilon_{v}<0}\left(\left|\epsilon_{\nu}\right|-E_{\nu}\right)+\frac{\Delta^{\prime 2}}{G} .
\end{aligned}
$$

Assuming a symmetric distribution of levels around the Fermi energy, the above expression becomes

$$
E_{\text {corr }}=2 \sum_{\nu>0 ; \epsilon_{v}>0}\left(\epsilon_{v}-E_{v}\right)+\frac{\Delta^{\prime 2}}{G} .
$$

It proves useful to express the ground-state energy in the term of occupation probabilities and potential interaction energy, namely as (see term $U$ Eq. (G.11) of Ref. [10])

$$
E_{\mathrm{gs}}=\sum_{\nu>0} 2 \epsilon_{\nu} V_{\nu}^{\prime 2}-\frac{\Delta^{\prime 2}}{G} .
$$

Using the expression for $V_{v}^{\prime 2}$,

$$
E_{\mathrm{gs}}=\sum_{\nu>0} \epsilon_{\nu}\left(1-\frac{\epsilon_{\nu}}{E_{v}}\right)-\frac{\Delta^{\prime 2}}{G}
$$

which may be rewritten as

$$
\begin{aligned}
E_{\mathrm{gs}} & =\sum_{\nu>0} \epsilon_{\nu}\left(1-\frac{\epsilon_{\nu}}{E_{\nu}}-\frac{E_{v}}{\epsilon_{\nu}}+\frac{E_{\nu}}{\epsilon_{v}}\right)-\frac{\Delta^{\prime 2}}{G} \\
& =\sum_{\nu>0}\left(\epsilon_{\nu}-E_{\nu}\right)+\sum_{\nu>0}\left(E_{\nu}-\frac{\epsilon_{v}^{2}}{E_{v}}\right)-\frac{\Delta^{\prime 2}}{G} \\
& =\sum_{\nu>0}\left(\epsilon_{\nu}-E_{\nu}\right)+\sum_{\nu>0} \frac{\Delta^{\prime 2}}{E_{v}}-\frac{\Delta^{\prime 2}}{G} \\
& =\sum_{\nu>0}\left(\epsilon_{v}-E_{\nu}\right)+2 \frac{\Delta^{\prime 2}}{G}-\frac{\Delta^{\prime 2}}{G} \\
& =\sum_{\nu>0}\left(\epsilon_{\nu}-E_{\nu}\right)+\frac{\Delta^{\prime 2}}{G},
\end{aligned}
$$

which coincides with (A66).

\section{APPENDIX B: PAIRING ROTATIONAL BAND WAVE FUNCTION}

Let us start by defining the operator inducing a gauge transformation. In keeping with the fact that the generator of such a transformation is the particle number operator, we deal with rotations in a two-dimensional space. 
The operator inducing rotation in pairspin space about the gauge $z$ axis is given as $e^{-2 i s_{z}(v) \phi}$. The representation of this operator in pairspin space is

$$
\left(\begin{array}{cc}
e^{-i \phi} & 0 \\
0 & e^{i \phi}
\end{array}\right)
$$

This operator converts the state $|\phi=0\rangle_{v}=\left(\begin{array}{c}V_{v}^{\prime} \\ U_{v}^{\prime}\end{array}\right)$ into the state rotated by an angle $\phi$ [cf. Eqs. (A40) and (A41)]:

$$
\left(\begin{array}{cc}
e^{-i \phi} & 0 \\
0 & e^{i \phi}
\end{array}\right)\left(\begin{array}{c}
V_{v}^{\prime} \\
U_{v}^{\prime}
\end{array}\right)=\left(\begin{array}{c}
e^{-i \phi} V_{v}^{\prime} \\
e^{i \phi} U_{v}^{\prime}
\end{array}\right)
$$

In the following we use the operator $\mathcal{G}_{v}(\phi)=e^{-i \phi N_{v}}$ which differs from $e^{-2 i \phi s_{z}(v)}$ only by an overall phase $e^{i \phi}$. Its representation in pairspin space is given by

$$
\left(\begin{array}{cc}
e^{-2 i \phi} & 0 \\
0 & 1
\end{array}\right)
$$

and its action on $|\phi=0\rangle_{v}=\left(\begin{array}{c}V_{v}^{\prime} \\ U_{v}^{\prime}\end{array}\right)$ is given by

$$
\left(\begin{array}{cc}
e^{-2 i \phi} & 0 \\
0 & 1
\end{array}\right)\left(\begin{array}{c}
V_{v}^{\prime} \\
U_{v}^{\prime}
\end{array}\right)=\left(\begin{array}{c}
e^{-2 i \phi} V_{v}^{\prime} \\
U_{v}^{\prime}
\end{array}\right)
$$

producing the phase difference characterizing the rotated, $|\mathrm{BCS}(\phi)\rangle$ state [cf. Eq. (A51)]. Calculating the average value of $P$ in the (rotated) state $\mathcal{G}(\phi)|\phi=0\rangle_{\nu}$, that is $\alpha_{0}(v)={ }_{\nu}\langle\phi=$ $\left.0\left|\mathcal{G}^{-1}(\phi) P \mathcal{G}(\phi)\right| \phi=0\right\rangle_{\nu}$, one finds

$$
\left(\begin{array}{ll}
e^{2 i \phi} V_{v}^{\prime} & U_{v}^{\prime}
\end{array}\right)\left(\begin{array}{ll}
0 & 0 \\
1 & 0
\end{array}\right)\left(\begin{array}{c}
e^{-2 i \phi} V_{v}^{\prime} \\
U_{v}^{\prime}
\end{array}\right)=e^{-2 i \phi} U_{v}^{\prime} V_{v}^{\prime} .
$$

We can also calculate the same average value by rotating the operator (Heisenberg representation), rather than acting on the state. The rotated operator is given by $\mathcal{G}_{\nu}^{\dagger}(\phi) P_{\nu}(\phi=$ $0) \mathcal{G}_{v}(\phi)$. The action of $\mathcal{G}_{v}^{\dagger}(\phi) P_{v}(\phi=0) \mathcal{G}_{v}(\phi)$ transforms the initial operator in the intrinsic frame $P_{v}(\phi=0)$, in which it has the average value $\alpha_{0}^{\prime}(v)=U_{v}^{\prime} V_{v}^{\prime}$ into the laboratory frame, in which its average value is $\alpha_{0}(v)=\alpha_{0}^{\prime}(v) e^{-2 i \phi}$. The inverse transformation, from the laboratory into the intrinsic frame, is effected by the operator $\left(\mathcal{G}_{v}^{\dagger}(\phi) P_{v}(\phi) \mathcal{G}_{v}(\phi)\right)^{-1}=$ $\mathcal{G}_{v}(\phi) P_{v}(\phi) \mathcal{G}^{\dagger}(\phi)$. In what follows, we use $P_{v}^{\prime} \equiv P_{v}(\phi=0)$ and $P_{v} \equiv P_{v}(\phi)$.

Similar considerations can be applied to the many-body wave functions. Let us consider a wave function

$$
\Psi_{\mathcal{K}}=a_{1}^{\dagger} a_{2}^{\dagger} \cdots a_{N_{0}}^{\dagger}|0\rangle
$$

with a fixed number of particles. Let us now apply $\mathcal{G}(\phi)$ to the creation operator

$$
a_{\nu}^{\prime \dagger}=\mathcal{G}(\phi) a_{\nu}^{\dagger} \mathcal{G}^{-1}(\phi)=e^{-i \phi} a_{\nu}^{\dagger},
$$

$a^{\prime \dagger}$ being referred to the intrinsic, body-fixed reference system (see Fig. 14). Thus,

$$
\begin{aligned}
\Psi_{\mathcal{K}}(\phi) & =e^{i N_{0} \phi} a_{1}^{\prime \dagger} a_{2}^{\prime \dagger} \cdots a_{N_{0}}^{\prime \dagger}|0\rangle \\
& =e^{i N \phi} \Psi_{K^{\prime}}(\phi=0), \\
\Psi_{\mathcal{K}^{\prime}}(\phi=0) & =a_{1}^{\prime \dagger} a_{2}^{\prime \dagger} \cdots a_{N_{0}}^{\prime \dagger}|0\rangle .
\end{aligned}
$$

In keeping with the fact that

$$
\begin{aligned}
-i \frac{\partial \Psi_{\mathcal{K}}(\phi)}{\partial \phi} & =-i \times i N_{0} e^{i N \phi} \Psi_{\mathcal{K}^{\prime}}(\phi=0) \\
& =N_{0} \Psi_{\mathcal{K}}(\phi), \\
N & =-i \frac{\partial}{\partial \phi}, \quad N \Psi_{\mathcal{K}}(\phi)=N_{0} \Psi_{\mathcal{K}}(\phi) .
\end{aligned}
$$

This is equivalent to saying that

$$
\begin{aligned}
{[\phi, N] \psi } & =(\phi N-N \phi)=\phi\left(-i \frac{\partial}{\partial \phi} \psi\right)+i \frac{\partial}{\partial \phi}(\phi \psi) \\
& =i \psi ;
\end{aligned}
$$

i.e., $[\phi, N]=i$. The BCS wave function can then be written in the intrinsic body-fixed frame as

$$
\left.\left|\operatorname{BCS}(\phi=0)>_{\mathcal{K}^{\prime}} \sim \prod_{\nu} \alpha_{\nu}\right| 0\right\rangle \sim \prod_{\nu>0} \alpha_{\nu} \alpha_{\bar{\nu}}|0\rangle .
$$

The corresponding normalized wave function is then

$$
\begin{aligned}
|\mathrm{BCS}(\phi=0)\rangle_{\mathcal{K}^{\prime}} & =\prod_{\nu>0}\left(U_{v}^{\prime}+V_{\nu}^{\prime} a_{\nu}^{\prime \dagger} a_{\bar{v}}^{\prime \dagger}\right)|0\rangle=\prod_{\nu}\left(U_{\nu}^{\prime}+e^{-2 i \phi} V_{\nu}^{\prime} a_{\nu}^{\dagger} a_{\bar{\nu}}^{\dagger}\right)|0\rangle \\
& =|\operatorname{BCS}(\phi)\rangle_{\mathcal{K}}=\left(\prod_{\nu>0} U_{v}^{\prime}\right)\left(1+\frac{e^{-2 i \phi}}{1 !} \sum_{\nu>0} c_{\nu} a_{\nu}^{\dagger} a_{\bar{\nu}}^{\dagger}+\frac{e^{-4 i \phi}}{2 !}\left(\sum_{\nu>0} c_{\nu} a_{\nu}^{\dagger} a_{\bar{\nu}}^{\dagger}\right)^{2}+\cdots\right)|0\rangle,
\end{aligned}
$$

where $c_{v}=V_{v}^{\prime} / U_{v}^{\prime}$. Thus,

$$
\begin{aligned}
\left|N_{0}\right\rangle & =\int d \phi e^{i N_{0} \phi}|\operatorname{BCS}(\phi=0)\rangle_{\mathcal{K}^{\prime}}=\int d \phi e^{i N_{0} \phi}|\operatorname{BCS}(\phi)\rangle_{\mathcal{K}} \\
& =\left(\Pi_{\nu>0} U_{\nu}^{\prime}\right) \int d \phi e^{i N_{0} \phi}\left(1+\cdots+\frac{e^{-i N \phi}}{(N / 2) !}\left(\sum_{\nu>0} c_{\nu} a_{\nu}^{+} a_{\bar{v}}^{+}\right)^{N / 2}+\cdots\right)|0\rangle \sim\left(\sum_{\nu>0} c_{\nu} a_{\nu}^{\dagger} a_{\bar{\nu}}^{\dagger}\right)^{N_{0} / 2}|0\rangle .
\end{aligned}
$$


It is of notice that the factor $e^{i N_{0} \phi}$ above is equivalent to the transformation coefficient $e^{i p q}$ between $p$ and $q$ representations. Finally, the members of a pairing rotational band are described by the states,

$$
\left|N_{0}\right\rangle \sim\left(\sum_{\nu>0} c_{\nu} a_{\nu}^{\dagger} a_{\bar{\nu}}^{\dagger}\right)^{N_{0} / 2}|0\rangle .
$$

Now, from this relation it becomes clear that the Cooper pair wave function,

$$
|\tilde{0}\rangle=\sum_{\nu>0} c_{\nu} a_{\nu}^{\dagger} a_{\bar{\nu}}^{\dagger}|0\rangle
$$

is to be interpreted to be valid for values of $\varepsilon_{v}$ close to $\varepsilon_{F}$; otherwise, one risks not being able to normalize it $\left(U_{v} \rightarrow 0\right.$ for $\varepsilon_{v} \ll \varepsilon_{F}<0$ for deeply bound occupied states). This is in keeping with the fact that pair condensation in general, and nuclear superfluidity in particular, are associated with a modification of the Fermi surface within a narrow band around it $\left(\varepsilon_{F} \pm \Delta\right)$. In other words, pairspin alignment as described by BCS implies that something unique takes place in the long wavelength limit of the spectrum, namely the appearance of a coherent state with almost [aside from the weak $\left(\varepsilon_{v} \approx \varepsilon_{F}\right)$ dealignment introduced by $H_{\mathrm{sp}}$ ] perfect phase coherence. This state behaves essentially semiclassically, and its properties can hardly depend on the ultraviolet behavior of the system. In other words, $E_{\text {cutoff can be set to include only the valence }}$ single-particle shells, adjusting $G$ to reproduce the value of the pairing gap. Within this context, see also the discussion in Sec. IV in connection with Fig. 8 and Tables I and II.

In the case of a single $j$ shell (see, e.g., Ref. [10], Appendix I),

$$
V^{\prime}=\sqrt{\frac{N}{2 \Omega}}, \quad U=\sqrt{1-\frac{N}{2 \Omega}} ;
$$

thus,

$$
U^{\prime} V^{\prime}=\sqrt{\frac{N}{2 \Omega}\left(1-\frac{N}{2 \Omega}\right)},
$$

while

$$
\frac{V^{\prime}}{U^{\prime}}=\frac{\sqrt{\frac{N}{2 \Omega}}}{\sqrt{1-\frac{N}{2 \Omega}}}=\sqrt{\frac{N}{2 \Omega-N}} .
$$

For a number of particles considerably smaller than the full degeneracy of the single-particle subspace in which nucleons can correlate, that is for $N \ll 2 \Omega$, one can write

$$
U^{\prime} V^{\prime}=\sqrt{\frac{N}{2 \Omega}\left(1-\frac{N}{2 \Omega}\right)} \approx \sqrt{\frac{N}{2 \Omega}},
$$

and

$$
\begin{aligned}
\frac{V^{\prime}}{U^{\prime}} & =\sqrt{\frac{N}{2 \Omega} \frac{1}{\left(1-\frac{N}{2 \Omega}\right)}} \\
& \approx \sqrt{\frac{N}{2 \Omega}}\left(1+\frac{N}{4 \Omega}\right) \approx \sqrt{\frac{N}{2 \Omega}} \approx U^{\prime} V^{\prime}
\end{aligned}
$$

Consequently,

$$
|\tilde{0}\rangle \approx \sum_{\nu>0} U_{\nu}^{\prime} V_{\nu}^{\prime} a_{\nu}^{\dagger} a_{\bar{\nu}}^{\dagger}|0\rangle,
$$

in keeping with Eqs. (15b) and (31).

In what follows we work out some relations which are useful to calculate expectation values in the $|\mathrm{BCS}\rangle$ state.

Making use of (B7) (i.e., $a_{\mu}^{\prime \dagger}=\mathcal{G} a_{\mu}^{\dagger} \mathcal{G}^{-1}=e^{-i \phi} a_{\mu}^{\dagger}$ ) one can write

$$
a_{\mu}^{\dagger}=e^{i \phi} a_{\mu}^{\prime \dagger}=e^{i \phi} \begin{cases}a_{\nu}^{\prime \dagger} & (\mu=v), \\ a_{\bar{v}}^{\prime \dagger} & (\mu=\bar{v})\end{cases}
$$

thus,

$$
a_{\mu}=e^{-i \phi} a_{\mu}^{\prime}=e^{-i \phi} \begin{cases}a_{v}^{\prime} & (\mu=v), \\ a_{\bar{v}}^{\prime} & (\mu=\bar{v}) .\end{cases}
$$

Making use of Eqs. (1b) and (2), one can write

$$
\alpha_{\mu}^{\dagger}= \begin{cases}U_{\nu}^{\prime} a_{\nu}^{\prime \dagger}-V_{\nu}^{\prime} a_{\bar{v}}^{\prime} & (\mu=v), \\ U_{\nu}^{\prime} a_{\bar{v}}^{\prime \dagger}+V_{\nu}^{\prime} a_{v}^{\prime} & (\mu=\bar{v}),\end{cases}
$$

in keeping with the fact that $U_{\mu}^{\prime}$ and $V_{\mu}^{\prime}$ are real $c$-numbers, and thus $U_{\mu}^{\prime}=U_{\bar{\mu}}^{\prime}$ and $V_{\mu}^{\prime}=V_{\bar{\mu}}^{\prime}$, and, consequently, $U_{\bar{\nu}}=U_{\nu}$ and $V_{\bar{v}}=V_{\nu}$, as well as the fact that $a_{\bar{\nu}}=-a_{\nu}$, a consequence of the antiunitary character of the time-reversal operator. Within this context it is of notice that the intrinsic property of a nucleon of being in a state with quantum numbers $(j, m)$ or $(j,-m)$ does not, of course, affect the gauge angle of rotation $(2 \phi$, see also Fig. 11) defining the intrinsic (body-fixed) frame of reference with respect to the laboratory system. Taking the Hermitian conjugate of the second case of Eq. (B26), one obtains

$$
\alpha_{\bar{v}}=U_{\nu}^{\prime} a_{\bar{v}}^{\prime}+V_{\nu}^{\prime} a_{\nu}^{\prime \dagger} .
$$

Multiplying the first entry of Eq. (B26) by $U_{v}^{\prime}$ and Eq. (B27) by $V_{\nu}^{\prime}$, one obtains

$$
\begin{aligned}
U_{v}^{\prime} \alpha_{v}^{\dagger} & =U_{v}^{\prime 2} a_{v}^{\prime \dagger}-V_{v}^{\prime} U_{v}^{\prime} a_{\bar{v}}^{\prime}, \\
V_{v}^{\prime} \alpha_{\bar{v}} & =V_{v}^{\prime} U_{v}^{\prime} a_{\bar{v}}^{\prime}+V_{v}^{\prime 2} a_{v}^{\prime \dagger}
\end{aligned}
$$

Summing these two expressions and making use of Eq. (3), one obtains

$$
a_{v}^{\prime \dagger}=U_{\nu}^{\prime} \alpha_{\nu}^{\dagger}+V_{\nu}^{\prime} \alpha_{\bar{v}}
$$

which is equivalent to

$$
a_{v}^{\dagger}=U_{\nu} \alpha_{\nu}^{\dagger}+V_{v}^{*} \alpha_{\bar{v}}
$$

Similarly, multiplying the Hermitian conjugate of the first entry of Eq. (B26) by $V_{v}^{\prime}$ and the second entry by $U_{v}^{\prime}$ and subtracting the resulting expressions leads to

$$
a_{\bar{v}}^{\prime \dagger}=U_{v}^{\prime} \alpha_{\bar{v}}^{\dagger}-V_{\nu}^{\prime} \alpha_{v}
$$

which is equivalent to

$$
a_{\bar{\nu}}^{\dagger}=U_{\nu} \alpha_{\bar{\nu}}^{\dagger}-V_{\nu}^{*} \alpha_{\nu}
$$


One then obtains

$$
\begin{aligned}
& P^{\dagger}=\sum_{\nu>0} a_{\nu}^{\dagger} a_{\bar{v}}^{\dagger} \\
& =\sum_{\nu>0}\left\{U_{\nu}^{2} \alpha_{\nu}^{\dagger} \alpha_{\bar{\nu}}^{\dagger}-\left(V_{v}^{*}\right)^{2} \alpha_{\bar{\nu}} \alpha_{\nu}\right. \\
& \left.-U_{v} V_{v}^{*}\left(\alpha_{v}^{\dagger} \alpha_{v}+\alpha_{\bar{\nu}}^{\dagger} \alpha_{\bar{v}}\right)+U_{v} V_{v}^{*}\right\}, \\
& P=\sum_{\nu>0} a_{\bar{\nu}} a_{\nu} \\
& =\sum_{\nu>0}\left\{\left(U_{\nu}^{*}\right)^{2} \alpha_{\bar{\nu}} \alpha_{\nu}-V_{\nu}^{2} \alpha_{\nu}^{\dagger} \alpha_{\bar{\nu}}^{\dagger}\right. \\
& \left.-U_{v}^{*} V_{\nu}\left(\alpha_{\nu}^{\dagger} \alpha_{\nu}+\alpha_{\bar{\nu}}^{\dagger} \alpha_{\bar{\nu}}\right)+U_{v}^{*} V_{v}\right\} ;
\end{aligned}
$$

thus,

$$
\begin{aligned}
\alpha_{0} & =\langle\mathrm{BCS}|P| \mathrm{BCS}\rangle=\sum_{\nu>0} U_{v}^{*} V_{v} \\
& =e^{-2 i \phi} \sum_{\nu>0} U_{v}^{\prime} V_{v}^{\prime}=e^{-2 i \phi} \alpha_{0}^{\prime} \\
& =\left(\sum_{\nu>0} U_{v} V_{v}^{*}\right)^{*}=\left\langle\mathrm{BCS}\left|P^{\dagger}\right| \mathrm{BCS}\right\rangle^{*} .
\end{aligned}
$$

Summing up,

$$
\alpha_{0}=\sum_{\nu>0} U_{v}^{*} V_{\nu}
$$

and

$$
\alpha_{0}^{\prime}=\sum_{\nu>0} U_{v}^{\prime} V_{v}^{\prime}
$$

leading to

$$
\Delta=G \alpha_{0}=e^{-2 i \phi} G \alpha_{0}^{\prime}=e^{-2 i \phi} \Delta^{\prime} .
$$

An alternative derivation of the above relations can be obtained by inserting the expressions of $a_{v}^{\prime \dagger}$ and $a_{\bar{v}}^{\prime \dagger}$ obtained from Eqs. (B28a) and (B29a) into

$$
P=\sum_{\nu>0} a_{\bar{\nu}} a_{\nu}=e^{-2 i \phi} \sum_{\nu>0} a_{\bar{\nu}}^{\prime} a_{\nu}^{\prime}=e^{-2 i \phi} P^{\prime},
$$

which leads to

$$
\begin{aligned}
P= & e^{-2 i \phi} \sum_{\nu>0}\left(U_{\nu}^{\prime} \alpha_{\bar{\nu}}-V_{\nu}^{\prime} \alpha_{\nu}^{\dagger}\right)\left(U_{\nu}^{\prime} \alpha_{\nu}+V_{\nu}^{\prime} \alpha_{\bar{\nu}}^{\dagger}\right) \\
= & e^{-2 i \phi} \sum_{\nu>0}\left\{U_{\nu}^{\prime 2} \alpha_{\bar{\nu}} \alpha_{\nu}-V_{\nu}^{\prime 2} \alpha_{\nu}^{\dagger} \alpha_{\bar{\nu}}^{\dagger}\right. \\
& \left.-U_{\nu}^{\prime} V_{\nu}^{\prime}\left(\alpha_{\nu}^{\dagger} \alpha_{\nu}+\alpha_{\bar{\nu}}^{\dagger} \alpha_{\bar{\nu}}\right)+U_{\nu}^{\prime} V_{\nu}^{\prime}\right\} .
\end{aligned}
$$

\section{APPENDIX C: GENERALIZED RIGIDITY IN GAUGE SPACE}

Generalized rigidity in gauge space implies that if one pushes, with the help of a field that changes the number of particles in two, one of the poles of a deformed system in gauge space (see, e.g., Fig. 14), a system that can be viewed as a wave packet in particle number, the other pole reacts rigidly to the push, and the system starts rotating as a whole (pairing rotational band). Similarly, if it was already in rotation it changes its rotational frequency from $\omega=\lambda\left(N_{0}\right) / \hbar$ to $\omega^{\prime}=$ $\lambda\left(N_{0} \pm 2\right) / \hbar, \lambda$ being the Lagrange multiplier which, in BCS theory, is closely connected with the particle number equation.

If the gauge space image is not sufficiently concrete to create a physical picture of the process, let us think of a quadrupole deformed nucleus whose intrinsic state is described in terms of the Nilsson intrinsic state. Making use of a proton beam which acts upon one of the poles, the system reacts as a whole and starts rotating with a frequency associated with one of the allowed values of the angular momentum, the transfer quantum corresponding to an energy inversely proportional to the moment of inertia.

One may argue that the reaction of the pole acted upon by the external field is not instantaneous but takes place only after an interval of time, compatible with the propagation of information in the nuclear medium, has elapsed. This parlance is not even wrong, as a wave function, in particular that describing the intrinsic ground state of a superfluid nucleus [see Eq. (12)], is not a matter function but a probability amplitude function ${ }^{5}$ with perfect phase coherence throughout. Within the quadrupole deformed nucleus analogy, generalized rigidity implies that the nucleus reacts rigidly as a whole to the action of the proton field acting on a pole, ${ }^{6}$ even if the moment of inertia of the associated rotational band is that of superfluid nuclear matter, and thus considerably smaller than the rigid moment of inertia.

The specific experiment to study the consequences (emergent properties) resulting from a spontaneous breaking of symmetry (e.g., of rotational invariance) is a probe which itself violates the symmetry in question. Now, while most of the devices we find in a well-equipped nuclear laboratory violate rotational invariance-think, for example, of a proton beamline defining a privileged orientation in 3D-space-one does not find many which violate gauge invariance. In other words, while rulers and goniometers defy empty space isotropy and homogeneity, one does not usually walk around with instruments which do not have a fixed number of particles.

This was the real importance of the Josephson effect (see Fig. 15), which provided a simple, and quantitative accurate answer to the question: How does one measures the gauge phase of a superconductor? The answer is, with the help of another superconductor displaying also an unknown but nonetheless well-defined gauge phase. Establishing a weak coupling (oxide layer) so that electrons can tunnel, one at a time, between the junction. If the first system can be viewed as a rotor in gauge space, the second one can equally well be represented in this way. Biasing the junction with a constant potential difference will lead to a two-rotor-coupled system (through pair transfer across the junction), rotating with frequencies which differ by $e \Delta V / \hbar=\left(\lambda_{1}-\lambda_{2}\right) / \hbar$. Such

\footnotetext{
${ }^{5}$ It is of notice that similar arguments are at the basis of the discussion of Bohr with Schrödinger and De Broglie (matter waves), let alone with Einstein (instantaneous) "information" propagation in connection with a single photon hitting a screen after having gone through a single slit of a box full of photons (see, e.g., Ref. [61]).

${ }^{6} \mathrm{To}$ avoid arguments such that the wavelength of the external hadronic field (beam) allows it to act on both poles, one can work in terms of a Gedanken experiment, in which the proton bombarding energy is about $1.6 \mathrm{GeV}(\lambda \approx 1 \mathrm{fm} \ll R)$.
} 

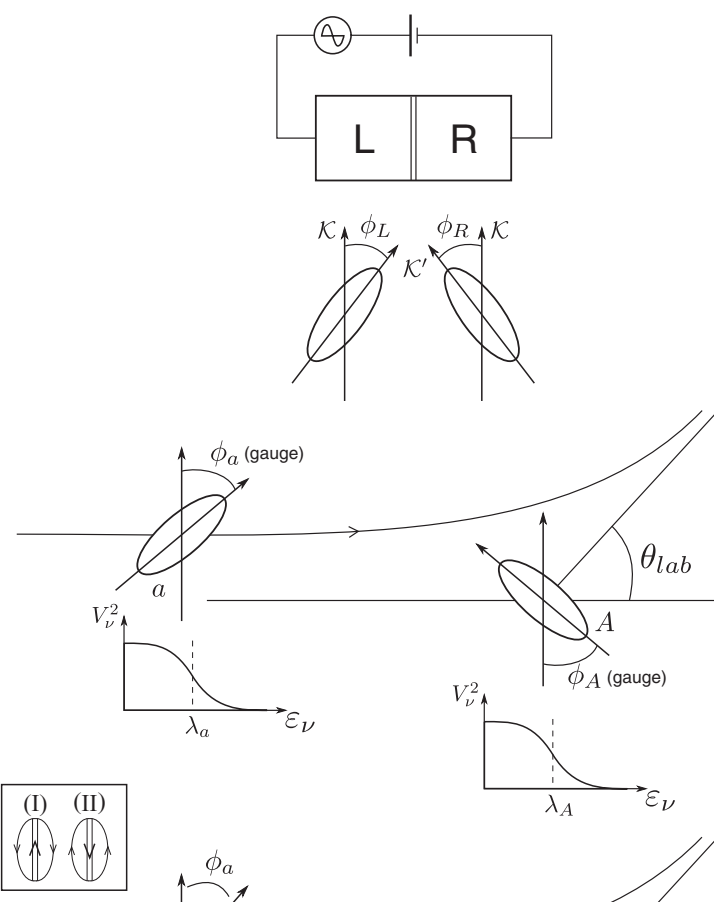

(b)

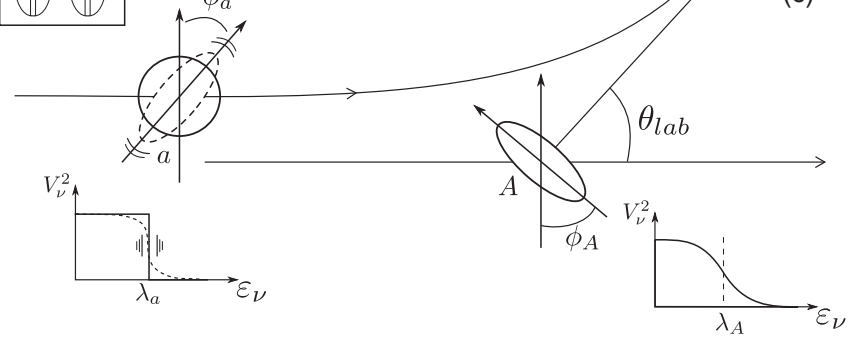

FIG. 15. (a) Josephson junction between left (L) and right (R) superconductors weakly coupled through a thin (dc-biased) oxide layer; (b) time-dependent nuclear Josephson junction established at about the distance of closest approach by the superfluid nuclei $a$ and $A$ in the reaction $a+A \rightarrow(a \pm n)+A(\mp n)$, where $n=0,1,2, \ldots$, is the number of Cooper pairs transferred in the process; (c) dynamical time-dependent Josephson junction between a closed-shell system $a$ displaying strong pairing vibrations (pair addition and pair removal mode) and a superfluid nucleus $A$. In the inset (left) the ground zero point fluctuations of the system $a$ associated with the pair addition (arrowed double line pointing up) and pair removal mode (arrowed double line pointing down) are shown.

a system will display a resonant behavior (alternating current with frequency $e \Delta V t / \hbar$ ) provided the rotor, deformed system picture in gauge space, is applicable. The fact that this effect provides the most accurate measure of $(e / \hbar)$ available testifies to the validity of the deformed rotor picture in gauge space associated with the BCS wave function (12).

It is sobering that this is so, in keeping with the fact that it was Bardeen who the most strenuous opponent to Josephson's ideas, even more so if one is reminded that the choice argument of such opposition was based on the fact that the pairing gap $\Delta=G \alpha_{0}$ vanishes at the junction. Now, the order parameter of BCS theory (as well as the justification of Cooper pair model) is $\alpha_{0}=\langle P\rangle=\left\langle P^{\dagger}\right\rangle^{*}$, namely the condensed pair field. Electrons may tunnel one at a time, without obliterating the validity of pair transfer, as the coherence length $\left(\xi=\hbar v_{F} / 2 \Delta\right)$ is much larger than typical junction dimensions, diverging at the junction (kind of an extreme ${ }^{11} \mathrm{Li}$-halo-like phenomenon (see, e.g., Refs. [16,62]) within the condensed-matter framework).

Within this scenario one can posit that, in the nuclear case, the equivalent of the Josephson junction device or better, the setup of an experiment that can measure differences in gauge phases allows for embodiments that are not possible within the field of condensed-matter physics. This is related to the fact that fluctuations in FMB systems in general and pairing vibrations in normal nuclei in particular are not only quantitatively but also qualitatively stronger than in condensed matter.

Consequently, not only a collision between two superfluid nuclei [see Fig. 15(b)] can be viewed as a time-dependent generalization of a Josephson junction [see Fig. 15(a)]. Also a normal-superfluid nuclear reaction can provide similar information, as a closed-shell system displays a very collective pairing vibrational spectrum which can be viewed as largeamplitude dynamical gauge symmetry violating mode [see Fig. 15(c)].

Let us consider the ground state of a (light) closed-shell system. Within the present discussion, it can be written as

$$
\widetilde{|a(\mathrm{gs})\rangle}=\alpha|a(\mathrm{gs})\rangle+\beta|(a-2)(\mathrm{gs}) \otimes(a+2)(\mathrm{gs})\rangle
$$

where $|(a-2)(\mathrm{gs})\rangle$ and $|(a+2)(\mathrm{gs})\rangle$ are the pair removal and pair addition modes, while $\alpha^{2}+\beta^{2}=1$ is the normalization condition. In other words, the closed-shell system is part of the time in states with two more [see (I) inset Fig. 15] or two less correlated nucleons [see (II) inset Fig. 15]. The two-hole uncorrelated states which, arguably, ensure particle number correlation, in fact describes the ground-state correlations (backwardsgoing amplitudes; see, e.g., Table III $Y$ amplitudes as well as insets in Fig. 3) associated with the pair addition mode which, dynamically, deforms the nucleus in gauge space defining a transient, privileged orientation. The same can be said concerning the two-particle uncorrelated system shown in the inset (II) of Fig. 15(c).

Let us now return to the analogy with quadrupole rotational and vibrational bands. Going away from closed-shell nuclei in medium heavy systems the energy of the first $2^{+}$state lowers in energy, the corresponding period becoming longer, the associated amplitudes larger (see, e.g., Ref. [10], Chap. 7). Eventually, after the quantal phase transition has taken place, the rotation of the system as a whole can be viewed as a very low-frequency quadrupole vibrational mode (for which the restoring force vanishes while inertia remains finite), which dynamically defines a privileged quadrupole deformation symmetry axis (and thus an associated set of Euler angles), which after each period changes orientation, with a frequency inversely proportional to the inertia of the mode.

In a similar way the state $(\mathrm{C} 1)$ defines dynamically a privileged orientation in gauge space which specifically can probe the corresponding static quantity of a superfluid target nucleus $A$ [see Fig. 15(c)], that is,

$$
\begin{aligned}
a+A & \rightarrow\left\{a((a+2)(\mathrm{gs}) \otimes(a-2)(\mathrm{gs}))+A\left(\sum_{N} c_{N}|N\rangle\right)\right\} \\
& \rightarrow\left\{\begin{array}{l}
(a-2)(\mathrm{gs})+(A+2)(\mathrm{gs}), \\
(a+2)(\mathrm{gs})+(A-2)(\mathrm{gs}),
\end{array}\right.
\end{aligned}
$$


where $A\left(\sum_{N} c_{N} \mid N\right)$ labels the ground state of a superfluid nucleus, e.g., of ${ }^{120} \mathrm{Sn}$ which can be viewed as a wave packet in neutron number, the scattering state within curly brackets being a virtual set of states each displaying a dynamical or a static privileged orientation in gauge space and thus a gauge phase. A particular embodiment of such a reaction can be

$$
{ }_{3}^{9} \mathrm{Li}_{6}+{ }_{50}^{120} \mathrm{Sn}_{70} \rightarrow\left\{\begin{array}{l}
{ }_{3}^{7} \mathrm{Li}_{4}+{ }_{50}^{122} \mathrm{Sn}_{72} \\
{ }_{3}^{11} \mathrm{Li}_{8}+{ }_{50}^{118} \mathrm{Sn}_{68}
\end{array}\right.
$$

in keeping with the fact that $N=6$ corresponds to a (parity inversion) magic number, $\left|{ }^{9} \mathrm{Li}(\mathrm{gs})\right\rangle$ and $\left|{ }^{11} \mathrm{Li}(\mathrm{gs})\right\rangle$ being the pair removal and pair addition modes of ${ }^{9} \mathrm{Li}$ (see Refs. [19,63]).

\section{APPENDIX D: ROBUSTNESS OF THE TWO-PARTICLE TRANSFER CROSS SECTION ESTIMATES}

The nuclear superfluid phase, and associated pairing rotational band behaves, because of its ODLRO essentially as a classical (coherent) state. This is the basic reason that is at the basis of the results displayed in Fig. 7 and Table IV, that is the remarkable quantitative accuracy with which theory provide an overall account of the absolute value of the experimental findings.

There is, however, a second reason for the higher accuracy with which one can, in principle, predict absolute twonucleon transfer cross sections, as compared with one-nucleon transfer cross sections, or, simply (although, arguably, less well defined, see, e.g., Refs. [5,64,65]), absolute values of the single-particle spectroscopic factors. This is connected with the ambiguity and eventual lack of consistency of empirically determined optical parameters. The first type of limitations is typically connected with the fact that the depth of the real part of the optical potential can have different, commensurable values, all leading to the same phase shift (e.g., so-called Igo's ambiguity [66]). Concerning the second point (consistency), one is reminded of the fact that the real and the imaginary part of the optical potentials $(U+i W)$ controlling off - and on - the energy shell processes are the real and imaginary part of the nuclear mass operator (sum of polarization and correlation contributions), referred to, also, as the nuclear dielectric function [5]. Consequently, $U$ and $W$ must fulfill the Kramers-Kronig dispersion relation (cf. Refs. [67,68]; see also Ref. [5]). This is a bidirectional mathematical relation, connecting the real and imaginary parts of any complex function that is analytic in the upper half of the complex plane. The Kramers-Kronig is a rather fundamental relation, in that it is strictly related to causality. Summing up, the real and imaginary parts of the optical potentials empirically determined from a global elastic scattering fitting, should respect the above mentioned dispersion relation. This condition is only marginally fulfilled in a number of cases, thus introducing uncertainties difficult to control. In keeping with the fact that, theoretically, $U(r)$ results from the convolution of the nucleon-nucleon interaction with the nuclear density $\rho(r)$ and that this quantity is best known around the nuclear surface, in a similar way in which $W(r)$ receives important contributions from the interweaving of single-particle motion and collective surface vibrations, one can posit that the lack of consistency between $U$ and $W$ is likely to be more serious for values of $r$ larger and smaller than the nuclear radius (i.e., $r>R_{0}$ and $r<R_{0}$ ). The phase coherence of the nuclear condensate implies that Cooper pair wave functions, closely related to the effective two-particle transfer nuclear form factors, are rather compact objects, essentially concentrated on the nuclear surface (see, e.g., Fig. 8). Consequently, the main contribution to the absolute two-particle differential cross section arises from values of $r \approx R_{0}$ (nuclear surface), region in which the optical potential is best known. However, the form factor associated with single-particle transfer is a standard mean-field wave function (in any case as far as the main peak of the single-particle strength function-one-pole approximation-is concerned). Consequently, the absolute one-nucleon transfer differential cross section can depend, in an important way, on the knowledge of the optical potential inside the nuclear volume.

The above discussion can also be related to the fluctuationdissipation theorem (cf., e.g., Refs. $[69,70]$ ), closely related to the Kramers-Kronig dispersion relation. While it is true that single-particle motion emerges from the same features leading to collective nuclear vibrations [60] - the independent particle shell structure being a result of a collective and concerted motion of all the nucleons allowing free motion to each single of them, and letting themselves be felt through the pushing and pulling only when a particle tries to leave the system, forcing it to bounce elastically off the nuclear surface-the picture becomes less stable as soon as the particle-vibration coupling strength is switched on, leading to real, dressed particles (mechanism also contributing to the imaginary part of the optical potential). Within this context one can posit that the associated single-particle strength function in general, and thus its centroid and width in particular, depend on a delicate interplay of spin- and non-spin-flip matrix elements, large and small energy denominators, and the like. However, the strength function associated with a Cooper pair, being a coherent object behaving almost semiclassically, represents much less of the above-mentioned inhomogeneous damping phenomena.

Summing up, even without totally consistent optical potentials in the Kramers-Kronig sense, it is likely that one can calculate the absolute value of the two-particle transfer cross section between members of pairing rotational (vibrational) bands with high accuracy. However, in the case of one-nucleon transfer processes, this possibility is likely to be restricted to the centroid and width of the (main peak) single-particle strength function, the integrated area being affected, as a rule, by little controllable, nonspecific background effects, difficult to estimate and/or remove. Such limitations will likely be more important in the case of strongly fragmented single-particle states.
[1] M. Mayer and J. Jensen, Elementary Theory of Nuclear Shell Structure (Wiley, New York, 1955).
[2] P. Cottle, Nature (London) 465, 430 (2010).

[3] K. L. Jones et al., Nature (London) 465, 454 (2010). 
[4] R. Haufler, L.-S. Wang, L. Chibante, C. Jin, J. Conceicao, Y. Chai, and R. Smalley, Chem. Phys. Lett. 179, 449 (1991).

[5] C. Mahaux, P. F. Bortignon, R. A. Broglia, and C. H. Dasso, Phys. Rep. 120, 1 (1985).

[6] N. N. Bogoliubov, Nuovo Cimento 7, 794 (1958).

[7] J. G. Valatin, Nuovo Cimento 7, 843 (1958).

[8] P. W. Anderson, Phys. Rev. 112, 1900 (1958).

[9] J. Bardeen, Phys. Rev. Lett. 9, 147 (1962).

[10] D. Brink and R. A. Broglia, Nuclear Superfluidity (Cambridge University Press, Cambridge, UK, 2005).

[11] D. R. Bès and R. A. Broglia, Nucl. Phys. 80, 289 (1966).

[12] R. A. Broglia, O. Hansen, and C. Riedel, Adv. Nucl. Phys. 6, 287 (1973), http://merlino.mi.infn.it/repository/ BrogliaHansenRiedel.pdf.

[13] R. S. Nikam and P. Ring, Phys. Rev. Lett. 58, 980 (1987).

[14] R. Nikam, P. Ring, and L. Canto, Phys. Lett. B 185, 269 (1987).

[15] Y. R. Shimizu, J. D. Garrett, R. A. Broglia, M. Gallardo, and E. Vigezzi, Rev. Mod. Phys. 61, 131 (1989).

[16] G. Potel, F. Barranco, E. Vigezzi, and R. A. Broglia, Phys. Rev. Lett. 105, 172502 (2010).

[17] R. A. Broglia, C. Riedel, and T. Udagawa, Nucl. Phys. A 169, 225 (1971).

[18] R. A. Broglia, C. Riedel, and T. Udagawa, Nucl. Phys. A 184, 23 (1972).

[19] R. A. Broglia, in Fifty Years of Nuclear BCS, edited by R. A. Broglia and V. Zelevinsky (World Scientific, Singapore, 2013), p. 643 .

[20] R. A. Broglia and A. Winther, Heavy Ion Reactions, 2nd ed. (Westview Press, Perseus Books, Boulder, CO, 2005).

[21] B. D. Josephson, Phys. Lett. 1, 251 (1962).

[22] J. Bardeen, Phys. Rev. 9, 147 (1962).

[23] M. H. Cohen, L. M. Falicov, and J. C. Phillips, Phys. Rev. Lett. 8, 316 (1962).

[24] T. Udagawa and D. K. Olsen, Phys. Lett. B 46, 285 (1973).

[25] W. S. Chien, C. H. King, J. A. Nolen, and M. A. M. Shahabuddin, Phys. Rev. C 12, 332 (1975).

[26] H. Segawa, K. I. Kubo, and A. Arima, Phys. Rev. Lett. 35, 357 (1975).

[27] M. Schneider, J. Burch, and P. Kunz, Phys. Lett. B 63, 129 (1976).

[28] N. B. de Takacsy, Phys. Rev. Lett. 31, 1007 (1973).

[29] N. De Takacsy, Nucl. Phys. A 231, 243 (1974).

[30] N. Hashimoto and M. Kawai, Prog. Theor. Phys. 59, 1245 (1978).

[31] K. I. Kubo and H. Amakawa, Phys. Rev. C 17, 1271 (1978).

[32] B. F. Bayman and J. Chen, Phys. Rev. C 26, 1509 (1982).

[33] K. Yagi, S. Kunori, Y. Aoki, K. Nagano, Y. Toba, and K. I. Kubo, Phys. Rev. Lett. 43, 1087 (1979).

[34] M. Igarashi, K. Kubo, and K. Yagi, Phys. Rep. 199, 1 (1991).

[35] M. B. Becha, C. O. Blyth, C. N. Pinder, N. M. Clarke, R. P. Ward, P. R. Hayes, K. I. Pearce, D. L. Watson, A. Ghazarian, M. D. Cohler, I. J. Thompson, and M. A. Nagarajan, Phys. Rev. C 56, 1960 (1997).

[36] M. Born, Z. für Physik 37, 863 (1926).

[37] Y. C. Tang and R. C. Herndon, Phys. Lett. 18, 42 (1965).

[38] P. Guazzoni, M. Jaskola, L. Zetta, A. Covello, A. Gargano, Y. Eisermann, G. Graw, R. Hertenberger, A. Metz, F. Nuoffer, and G. Staudt, Phys. Rev. C 60, 054603 (1999).

[39] P. Guazzoni, L. Zetta, A. Covello, A. Gargano, G. Graw, R. Hertenberger, H.-F. Wirth, and M. Jaskola, Phys. Rev. C 69, 024619, (2004).
[40] P. Guazzoni, L. Zetta, A. Covello, A. Gargano, B. F. Bayman, G. Graw, R. Hertenberger, H.-F. Wirth, and M. Jaskola, Phys. Rev. C 74, 054605 (2006).

[41] P. Guazzoni, L. Zetta, A. Covello, A. Gargano, B. F. Bayman, T. Faestermann, G. Graw, R. Hertenberger, H.-F. Wirth, and M. Jaskola, Phys. Rev. C 78, 064608 (2008).

[42] P. Guazzoni, L. Zetta, A. Covello, A. Gargano, B. F. Bayman, T. Faestermann, G. Graw, R. Hertenberger, H.-F. Wirth, and M. Jaskola, Phys. Rev. C 83, 044614 (2011).

[43] P. Guazzoni, L. Zetta, A. Covello, A. Gargano, B. F. Bayman, G. Graw, R. Hertenberger, H.-F. Wirth, T. Faestermann, and M. Jaskóla, Phys. Rev. C 85, 054609 (2012).

[44] G. Bassani, N. M. Hintz, C. D. Kavaloski, J. R. Maxwell, and G. M. Reynolds, Phys. Rev. 139, B830 (1965).

[45] G. Potel, F. Barranco, F. Marini, A. Idini, E. Vigezzi, and R. A. Broglia, Phys. Rev. Lett. 107, 092501 (2011).

[46] G. Potel, F. Barranco, F. Marini, A. Idini, E. Vigezzi, and R. A. Broglia, Phys. Rev. Lett. 108, 069904(E) (2012).

[47] H. An and C. Cai, Phys. Rev. C 73, 054605 (2006).

[48] S. Yoshida, Nucl. Phys. 33, 685 (1962).

[49] C. N. Yang, Rev. Mod. Phys. 34, 694 (1962).

[50] S. Galès, in Fifty Years of Nuclear BCS, edited by R. A. Broglia and V. Zelevinsky (World Scientific, Singapore, 2013), p. 419.

[51] A. Idini, F. Barranco, and E. Vigezzi, Phys. Rev. C 85, 014331 (2012).

[52] E. R. Flynn, G. J. Igo, and R. A. Broglia, Phys. Lett. B 41, 397 (1972).

[53] P. Bortignon, R. Broglia, and D. Bes, Phys. Lett. B 76, 153 (1978).

[54] F. Dönau, K. Hehl, C. Riedel, R. Broglia, and P. Federman, Nucl. Phys. A 101, 495 (1967).

[55] H. Barz, K. Hehl, C. Riedel, and R. Broglia, Nucl. Phys. A 126, 577 (1969).

[56] K. Wimmer et al., Phys. Rev. Lett. 105, 252501 (2010).

[57] R. A. Broglia, C. Riedel, and T. Udagawa, Nucl. Phys. A 135, 561 (1969).

[58] D. R. Bès, R. A. Broglia, G. G. Dussel, R. J. Liotta, and H. M. Sofía, Nucl. Phys. A 260, 1 (1976).

[59] D. R. Bès, R. A. Broglia, G. G. Dussel, R. J. Liotta, and H. M. Sofía, Nucl. Phys. A 260, 27 (1976).

[60] B. R. Mottelson, in International School of Physics "Enrico Fermi" Course XV, Nuclear Spectroscopy, edited by G. Racah (Academic Press, New York, 1962), p. 44.

[61] D. Lindley, Uncertainty, Einstein, Heisenberg, Bohr and the Struggle for the Soul of Science (Doubleday, New York, 2007).

[62] G. Potel and R. A. Broglia, in Fifty Years of Nuclear BCS, edited by R. A. Broglia and V. Zelevinsky (World Scientific, Singapore, 2013), p. 479.

[63] G. Potel, A. Idini, F. Barranco, E. Vigezzi, and R. Broglia, arXiv:0906.4298 [nucl-th].

[64] W. Dickhoff and D. Van Neck, Many-Body Theory Exposed! Propagator Description of Quantum Mechanics in Many-Body Systems (World Scientific, Singapore, 2005).

[65] B. Jennings, arXiv:1102.3721v1.

[66] G. Igo, Phys. Rev. 115, 1665 (1959).

[67] R. Kronig, J. Opt. Soc. Am. 12, 547 (1926).

[68] H. Kramers, Atti Cong. Int. Fis. (Transactions of Volta Centennial Congress) Commun. 2, 545, (1927).

[69] H. Nyquist, Phys. Rev. 32, 110, (1928).

[70] H. Callen and T. Welton, Phys. Rev. 83, 34 (1951). 